\title{
The WISSH quasars project
}

\section{Broad line region versus kiloparsec-scale winds}

\author{
G. Vietri ${ }^{1,2,3,4}$, E. Piconcelli ${ }^{1}$, M. Bischetti ${ }^{1,5}$, F. Duras ${ }^{6,1}$, S. Martocchia ${ }^{7}$, A. Bongiorno ${ }^{1}$, A. Marconi ${ }^{8,9}$, \\ L. Zappacosta ${ }^{1}$, S. Bisogni ${ }^{10}$, G. Bruni ${ }^{11}$, M. Brusa ${ }^{12,13}$, A. Comastri ${ }^{13}$, G. Cresci ${ }^{9}$, C. Feruglio ${ }^{14,1}$, \\ E. Giallongo ${ }^{1}$, F. La Franca ${ }^{6}$, V. Mainieri ${ }^{4}$, F. Mannucci ${ }^{9}$, F. Ricci ${ }^{6}$, E. Sani ${ }^{15}$, V. Testa ${ }^{1}$, F. Tombesi ${ }^{5,16,17}$, \\ C. Vignali ${ }^{12,13}$, and F. Fiore ${ }^{1}$
}

\footnotetext{
${ }^{1}$ INAF - Osservatorio Astronomico di Roma, Via Frascati 33, 00078 Monteporzio Catone, Italy e-mail: giustina.vietri@gmail.com

${ }^{2}$ Università degli Studi di Roma "La Sapienza", Piazzale Aldo Moro 5, 00185 Roma, Italy

${ }^{3}$ Excellence Cluster Universe, Technische Universität München, Boltzmannstr. 2, 85748 Garching, Germany

${ }^{4}$ European Southern Observatory, Karl-Schwarzschild-Str. 2, 85748 Garching bei München, Germany

5 Dipartimento di Fisica, Università degli Studi di Roma "Tor Vergata", Via della Ricerca Scientifica 1, 00133 Roma, Italy

${ }^{6}$ Dipartimento di Matematica e Fisica, Università degli Studi Roma Tre, Via della Vasca Navale 84, 00146 Roma, Italy

7 Astrophysics Research Institute, Liverpool John Moores University, 146 Brownlow Hill, Liverpool L3 5RF, UK

${ }^{8}$ Dipartimento di Fisica e Astronomia, Università di Firenze, Via G. Sansone 1, 50019 Sesto Fiorentino, Firenze, Italy

${ }^{9}$ INAF - Osservatorio Astrofisico di Arcetri, Largo E. Fermi 5, 50125 Firenze, Italy

${ }^{10}$ Harvard-Smithsonian Center for Astrophysics, 60 Garden St., Cambridge, MA 02138, USA

${ }^{11}$ INAF - Istituto di Astrofisica e Planetologia Spaziali, Via Fosso del Cavaliere 100, 00133 Rome, Italy

${ }_{12}$ Dipartimento di Fisica e Astronomia, Università degli Studi di Bologna, Via Gobetti 93/2, 40129 Bologna, Italy

13 INAF - Osservatorio Astronomico di Bologna, Via Gobetti 93/3, 40129 Bologna, Italy

${ }_{14}$ INAF - Osservatorio Astronomico di Trieste, Via G.B. Tiepolo 11, 34143 Trieste, Italy

${ }^{15}$ European Southern Observatory (ESO), Alonso de Cordova 3107, Vitacura, Santiago, Chile

${ }^{16}$ Department of Astronomy, University of Maryland, College Park, MD 20742 USA

${ }^{17}$ NASA/Goddard Space Flight Center, Code 662, Greenbelt, MD 20771 USA
}

Received 21 November 2017 / Accepted 8 February 2018

\begin{abstract}
Winds accelerated by active galactic nuclei (AGNs) are invoked in the most successful models of galaxy evolution to explain the observed physical and evolutionary properties of massive galaxies. Winds are expected to deposit energy and momentum into the interstellar medium (ISM), thus regulating both star formation and supermassive black hole (SMBH) growth. We undertook a multiband observing program aimed at obtaining a complete census of winds in a sample of WISE/SDSS selected hyper-luminous (WISSH) quasars (QSOs) at $z \approx 2-4$. We analyzed the rest-frame optical (i.e. LBT/LUCI and VLT/SINFONI) and UV (i.e. SDSS) spectra of 18 randomly selected WISSH QSOs to measure the SMBH mass and study the properties of winds both in the narrow line region (NLR) and broad line region (BLR) traced by blueshifted or skewed [OIII] and CIV emission lines, respectively. These WISSH QSOs are powered by SMBH with masses $\gtrsim 10^{9} M_{\odot}$ accreting at $0.4<\lambda_{\text {Edd }}<3.1$. We found the existence of two subpopulations of hyper-luminous QSOs characterized by the presence of outflows at different distances from the SMBH. One population (i.e. [OIII] sources) exhibits powerful [OIII] outflows, a rest-frame equivalent width (REW) of the CIV emission $R E W_{\mathrm{CIV}} \approx 20-40 \AA$, and modest CIV velocity $\operatorname{shift}\left(v_{\mathrm{CIV}}^{\text {peak }}\right)$ with respect to the systemic redshift $\left(v_{\mathrm{CIV}}^{\text {peak }} \lesssim 2000 \mathrm{~km} \mathrm{~s}^{-1}\right)$. The second population (i.e. Weak [OIII] sources), representing $\sim 70 \%$ of the analyzed WISSH QSOs, shows weak or absent [OIII] emission and an extremely large blueshifted CIV emission ( $v_{\text {CIV }}^{\text {peak }}$ up to $\sim 8000 \mathrm{~km} \mathrm{~s}^{-1}$ and $R E W_{\mathrm{CIV}} \lesssim 20 \AA$ ). We propose two explanations for the observed behavior of the strength of the [OIII] emission in terms of the orientation effects of the line of sight and ionization cone. The dichotomy in the presence of BLR and NLR winds could be likely due to inclination effects considering a polar geometry scenario for the BLR winds. In a few cases these winds are remarkably as powerful as those revealed in the NLR in the [OIII] QSOs $\left(\dot{E}_{\mathrm{kin}} \sim 10^{44-45} \mathrm{erg} \mathrm{s}^{-1}\right)$. We also investigated the dependence of these CIV winds on fundamental AGN parameters such as bolometric luminosity $\left(L_{\mathrm{Bol}}\right)$, Eddington ratio $\left(\lambda_{\mathrm{Edd}}\right)$, and UV-to-X-ray continuum slope $\left(\alpha_{\mathrm{OX}}\right)$. We found a strong correlation with $L_{\mathrm{Bol}}$ and an anti-correlation with $\alpha_{\mathrm{OX}}$ whereby the higher the luminosity, the steeper the ionizing continuum described by means of $\alpha_{\mathrm{OX}}$ and the larger the blueshift of the CIV emission line. Finally, the observed dependence $v_{\mathrm{CIV}}^{\text {peak }} \propto L_{\mathrm{Bol}}^{0.28} \pm 0.04$ is consistent with a radiatively-driven-winds scenario, where a strong UV continuum is necessary to launch the wind and a weakness of the X-ray emission is fundamental to prevent overionization of the wind itself.
\end{abstract}

Key words. galaxies: active - galaxies: nuclei - quasars: emission lines - quasars: general - quasars: supermassive black holes ISM: jets and outflows 


\section{Introduction}

Supermassive black holes (SMBHs) at the center of galaxies grow through the accretion of nearby matter and a fraction of the gravitational potential of the accreted mass is converted into radiation. In this case the SMBH is called an active galactic nucleus (AGN). The idea of a possible connection between the growth of the SMBH and the evolution of its host galaxy was put forward, once a correlation between black hole mass $\left(M_{\mathrm{BH}}\right)$ and the bulge luminosity was discovered (Kormendy \& Richstone 1995; Silk \& Rees 1998; Granato et al. 2004; Di Matteo et al. 2005; Menci et al. 2008). Since the energy released by the growth of the black hole can be larger than the binding energy of the galaxy bulge, the AGN can have an important effect on the evolution of its host galaxy. Indeed, fast winds can be launched from the accretion disk, collide with the interstellar medium (ISM), and drive powerful kiloparsec-scale outflows, which may sweep out the gas in the galaxy and then may be responsible for the regulation of the star formation and $\mathrm{BH}$ accretion (Zubovas \& King 2012; Faucher-Giguère \& Quataert 2012; Fabian 2012, see Fiore et al. 2017 for a detailed discussion).

Over the past few years efforts have been made to search for AGN outflows in different ISM phases. Evidence of radiativelydriven winds are observed at subparsec scale, in the innermost regions of the AGN through the detection of blueshifted, highly ionized Fe K-shell transitions, that is, ultra fast outflows (UFOs), with velocities $\sim 0.2$ c (King \& Pounds 2015; Tombesi et al. 2010, 2014; Gofford et al. 2013); at parsec scale via warm absorbers and broad absorption lines (BAL), moving at velocities up to $10000 \mathrm{~km} \mathrm{~s}^{-1}$ (King \& Pounds 2015; Tombesi et al. 2013); at kpc scale through different gas phases such as ionized gas, that is, broad asymmetric blueward [OIII] emission lines (Harrison et al. 2012, 2014; Cano-Díaz et al. 2012; Brusa et al. 2015; Carniani et al. 2015); neutral atomic gas such as NaID (Rupke \& Veilleux 2015), and molecular gas with velocities up to a few thousands $\mathrm{km} \mathrm{s}^{-1}$ (Feruglio et al. 2010; Cicone et al. 2014; Spoon et al. 2013). The investigation of the possible correlations of AGN properties over a wide range of spatial scales can give us more information about the nature of AGNs and the impact they have on their surroundings.

The most popular theoretical models of AGN-driven outflows (Faucher-Giguère \& Quataert 2012) suggest that the kinetic energy of the nuclear fast outflows is transferred to the ISM and drive the kpc-scale flows, in a likely energy-conserving scenario. This has been reflected in the observations of both UFO and molecular winds in two sources, Markarian 231 (Feruglio et al. 2015) and IRASF11119+13257 (Tombesi et al. 2015, but see Veilleux et al. 2017).

The high ionization lines, such as CIV $\lambda 1549 \AA$, are known to exhibit asymmetric profiles toward the blue-side, with the peak of the emission line blueshifted with respect to the low ionization lines, such as MgII or $\mathrm{H} \beta$ (Gaskell 1982; Sulentic et al. 2000 and Richards et al. 2011). This behavior cannot be ascribed to virialized motion but it can be interpreted in terms of outflowing gas, which possibly leads to biased $\mathrm{BH}$ mass estimates derived from the entire CIV emission line (Denney 2012; Coatman et al. 2017).

The blueshift of the CIV emission line can be a valuable tool to trace the winds in the broad line region (BLR). The CIV emission line typically shows a blueshift of $\sim 600 \mathrm{~km} \mathrm{~s}^{-1}$ up to $2000 \mathrm{~km} \mathrm{~s}^{-1}$ (Shen et al. 2011; Richards et al. 2002). The largest blueshifts have been discovered in an extreme quasar (QSO) population at $2.2 \lesssim z \lesssim 5.9$, the so-called weak line QSOs (hereafter WLQs), which exhibit weak or undetectable ultraviolet (UV) emission lines, that is, they are defined as QSOs with a rest-frame equivalent width (REW) of the CIV emission line of less than $10 \AA$ (Fan et al. 1999; Diamond-Stanic et al. 2009; Plotkin et al. 2010; Wu et al. 2011). The velocity shift of the CIV emission line correlates with several parameters of optical and ultraviolet emission lines. In particular it is part of 4D Eigenvector 1 parameter space (Boroson \& Green 1992; Sulentic et al. 2000), which also involves the broad Fe II emission, the equivalent width of the narrow [OIII] component, the width of the $\mathrm{H} \beta$ emission line, and the X-ray photon index. The physical driver of the $4 \mathrm{D}$ Eigenvector 1 is thought to be the Eddington ratio $\left(\lambda_{\text {Edd }}\right)$, since QSOs showing large blueshifts accrete at high rate, with $\lambda_{\text {Edd }} \geq$ 0.2 (Marziani et al. 2001; Boroson 2002; Shen \& Ho 2014).

As found by Marziani et al. (2016), these winds occurring in the BLR may affect the host galaxy. They studied a sample of QSOs with $L_{\mathrm{Bol}}=10^{45-48.1} \mathrm{erg} \mathrm{s}^{-1}$ at $0.9 \lesssim z \lesssim 3$ and found that the outflow kinetic power of these winds traced by the CIV emission line is comparable to the binding energy of the gas in a massive spheroid, underlining the importance of considering also these winds in the feedback scenario. It is therefore crucial to study such AGN-driven outflows at the golden epoch of AGN activity, for example, $1.5<z<3.5$. Furthermore, from theory and observations we know that the efficiency in driving energetic winds increases with AGN luminosity (Menci et al. 2008; Faucher-Giguère \& Quataert 2012), which brings out the need to investigate the properties of the most luminous QSOs.

We have undertaken a multiwavelength survey to investigate a sample of 86 Type-1 WISE/SDSS selected hyper-luminous AGNs $\left(L_{\mathrm{Bol}}>2 \times 10^{47} \mathrm{erg} \mathrm{s}^{-1}\right.$; Duras et al., in prep.), known as the WISSH sample (see Bischetti et al. 2017 for further details, Paper I hereafter; Duras et al. 2017). In Paper I we discussed the discovery of extreme kpc-scale outflows in five WISSH QSOs, traced via the blueshift of the [OIII] $\lambda 5007 \AA$ emission line. In this paper we report on the discovery of fast BLR winds (with velocities up to $8000 \mathrm{~km} \mathrm{~s}^{-1}$ ) traced by the blueshift of the CIV with respect to the systemic redshift, and their possible relation with the narrow line region (NLR) winds. We concentrate the analysis on the first 18 WISSH QSOs with rest-frame optical observations and UV archival data at $z \sim 2.2-3.6$. In Sect. 2, we present details about the observations and data reduction of proprietary data, such as LBT/LUCI1 and VLT/SINFONI spectra, and archival data from SDSS DR10. In Sect. 3, we outline the models used in our spectral analysis and present the results of the spectral fit, both for optical and UV spectra. In Sect. 4.1, we discuss the properties of the [OIII] and $\mathrm{H} \beta$ emission lines in the context of a scenario based on the orientation of our line of sight. Eddington ratios and $\mathrm{H} \beta$-based SMBH masses are presented in Sect. 4.2. We discuss the properties of the CIV emission line in Sect. 4.3, with particular emphasis on the blueshifted component and the mass outflow rate and kinetic power of the associated BLR winds in the WISSH QSOs, and their relation with NLR winds traced by [OIII]. In Sect. 5 we investigate the dependence of the CIV velocity shifts on fundamental AGN parameters such as $L_{\mathrm{Bol}}, \lambda_{\mathrm{Edd}}$, and the spectral index $\alpha_{\mathrm{OX}}$ defined as

$\alpha_{\mathrm{OX}}=\frac{\log \left(\mathrm{L}_{2 \mathrm{keV}} / \mathrm{L}_{2500 \AA}\right)}{\log \left(v_{2 \mathrm{keV}} / v_{2500 \AA}\right)}$

and representing the slope of a power law defined by the restframe monochromatic luminosities at $2 \mathrm{keV}\left(L_{2 \mathrm{keV}}\right)$ and $2500 \AA$ $\left(L_{2500 \AA}\right)$. Finally we summarize our findings in Sect. 6. Throughout this paper, we assume $H_{0}=71 \mathrm{~km} \mathrm{~s}^{-1} \mathrm{Mpc}^{-1}, \Omega_{\Lambda}=0.73$, and $\Omega_{m}=0.27$. 


\section{Observations and data reduction}

We have collected near-infrared (NIR) spectroscopic observations for 18 WISSH QSOs. Five of them have been discussed in Paper I, while the properties of an additional 13 QSOs, with LUCI1/LBT (ten objects) and VLT/SINFONI (three objects) observations are presented in the present paper. The coordinates, SDSS tenth data release (DR10) redshift, optical photometric data (Ahn et al. 2014), 2MASS NIR photometric data (Skrutskie et al. 2006), and color excess $E(B-V)$ derived from broadband SED fitting (Duras et al., in prep) of the WISSH QSOs analyzed here are listed in Table 1.

\subsection{LUCI/LBT observations}

The observations of ten WISSH QSOs were carried out with LUCI1, the NIR spectrograph and imager at the Large Binocular Telescope (LBT) located in Mount Graham, Arizona. We required medium resolution spectroscopic observations in longslit mode with a slit of 1 arcsec width, using the N1.8 camera. The gratings $150 \_\mathrm{K}_{\mathrm{s}}(R=4140)$ in the $K$ band and 210_zJHK $(R=7838)$ in $H$ band were used for objects at redshift bins $2.1<z<2.4$ and $3.1<z<3.6$, respectively. The wavelength ranges covered are $1.50-1.75 \mu \mathrm{m}$ and $1.95-2.40 \mu \mathrm{m}$ for the $H$ and $K$ bands, respectively. The observations were performed from April 2014 to March 2015, in two different cycles. The average on-site seeing of both cycles is comparable, $\sim 0.8$ arcsec (see Table 2 for further details). Observations of stars with known spectral type were taken during the observing nights to account for telluric absorption and flux calibration.

The data reduction was performed using IRAF tasks and IDL routines on both targets and standard stars. The reduced final frames are calibrated in wavelength and flux and are free from sky lines and telluric absorption. We used argon and neon arc-lamps for the wavelength calibration of J0801+5210, J0958+ 2827, $\mathrm{J} 1106+6400, \mathrm{~J} 1157+2724$, and $\mathrm{J} 1201+0116$; OH sky emission lines for the J1111+1336, J1236+6554, J1421+4633, and $\mathrm{J} 1422+4417$; and finally, argon and xenon arc-lamps for $\mathrm{J} 1521+5202$. For the lines' identification, a cubic spline was used to fit the pixel coordinates to the wavelengths provided. Analysis of the night sky lines indicated an uncertainty in the wavelength calibration of $\lesssim 20 \mathrm{~km} \mathrm{~s}^{-1}$. We performed an unweighted extraction of the 1D spectrum, using different apertures according to the spatial profile of each 2D spectrum, which was traced using a Chebyshev function of the 3rd order.

The sky subtraction was done by subtracting frames corresponding to different telescope pointings along the slit (ABBA method). To remove the atmospheric absorption features we used the IDL routine XTELLCOR_GENERAL (Vacca et al. 2003). This routine makes use of (i) the observed standard stars spectra, which are affected by telluric absorption as the targets, and (ii) a Vega model spectrum, useful to build up an atmosphericabsorption-free spectrum. As the magnitudes of the standard stars are also known, we calibrated the target spectra in absolute flux, with the same IDL routine.

\subsection{SINFONI observations}

Three QSOs were observed with Spectrograph for INtegral Field Observations in the Near Infrared (SINFONI), at the VLT installed at the Cassegrain focus of Unit Telescope 4 (UT4). The observations were performed in service mode during the period 093.A, with the adaptive optics (AO) - Laser Guide Star (LGS) mode, by which the atmospheric turbulence can be partially corrected. J1538+0855 and J2123-0050 were observed during the nights of Jul. 19 and Jul. 20 2014, while J2346-0016 was observed during Aug. 18 and Sept. 1 2014, as part of the program 093.A-0175. All the targets were observed within a field of view of $3^{\prime \prime} \times 3^{\prime \prime}$, with a spaxel scale of $0.05^{\prime \prime} \times 0.1^{\prime \prime}$. The final spatial resolution obtained thanks to the LGS correction is $\sim 0.3^{\prime \prime}$ for $\mathrm{J} 1538+0855$ and $\mathrm{J} 2346-0016$, and $\sim 0.2^{\prime \prime}$ for $\mathrm{J} 2123-0050$, as derived by the $\mathrm{H} \beta$ broad emission line. Given the redshift range of the QSOs, we used $K$ and $H$ filters to target the $\mathrm{H} \beta-[\mathrm{OIII}] \lambda 5007 \AA$ spectral region with a resolving power of $R \sim 4000$ and 3000, respectively. The data reduction was performed using ESO pipelines, in Reflex environment (Freudling et al. 2013). The background sky emission was removed with the IDL routine SKYSUB, using object frames taken at different telescope pointings (see Davies 2007). After the cube reconstruction, the flux calibration was performed with our own IDL routines, by means of telluric spectra acquired during the same nights as the targets. As a final step, we extracted a 1D spectrum for each source with a circular aperture of $1^{\prime \prime}$ diameter. This procedure does not allow us to have the spatial information, but we are interested in the integrated emission from different AGN components (i.e., BLR and NLR). The analysis of the spatiallyresolved kinematics of the [OIII] $\lambda 5007$ emission line will be presented in a forthcoming paper. Detailed information about SINFONI observations are listed in Table 3.

\subsection{SDSS archival data}

We retrieved the rest-frame UV spectrum of the 18 WISSH QSOs with NIR spectroscopic data, from the Sloan Digital Sky Survey (SDSS) DR10 public archive, in order to derive the properties of the CIV emission line. The SDSS survey uses a dedicated wide-field $2.5 \mathrm{~m}$ telescope (Gunn et al. 2006) at Apache Point Observatory in Southern New Mexico. The spectra were obtained from February 2001 to February 2012 with two spectrographs, SDSS-I and BOSS, with a resolving power of 1500 at $3800 \AA$ and 2500 at $9000 \AA$. The optical brightness of our objects, $15.6<r<18.25$, ensures data with an excellent signal-to-noise ratio $(>15)$ of the analyzed spectra.

\section{Spectroscopic analysis}

\subsection{Modeling of the $H \beta$ and [OIII] emission}

The visual inspection of the rest-frame optical spectra of the 13 WISSH QSOs taken with LBT/LUCI or VLT/SINFONI revealed the weakness of the [OIII] $\lambda \lambda 4959,5007 \AA$ doublet emission lines and the presence of a strong and complex FeII emission (see Appendix B). This combination makes the determination of [OIII] spectral parameters an extremely challenging task. In one case, that of J1422+4417, the LBT/LUCI1 bandwidth does not cover the [OIII] spectral region.

We developed a model based on the IDL package MPFIT (Markwardt 2009) in order to accurately infer the properties of the [OIII] and $\mathrm{H} \beta$ emission in these hyper-luminous QSOs. The NIR spectra were fitted with a spectral model which consists of:

$H \beta /[O I I I]$ core components associated with the NLR. Specifically, one Gaussian component for fitting the core of $\mathrm{H} \beta$ $4861 \AA$ line with the full width at half maximum $F W H M_{\mathrm{H} \beta}^{\text {core }} \leq$ $1000^{1} \mathrm{~km} \mathrm{~s}^{-1}$ plus one Gaussian profile for each component of the [OIII] $\lambda \lambda 4959,5007 \AA$ doublet. The FWHM of the [OIII]

\footnotetext{
1 The limit of $1000 \mathrm{~km} \mathrm{~s}^{-1}$ for the FWHM has been set in order to account for a possible subtle $\mathrm{H} \beta$ outflow component.
} 
Table 1. Properties of the WISSH QSOs considered in this paper.

\begin{tabular}{|c|c|c|c|c|c|c|c|c|c|c|c|c|}
\hline $\begin{array}{l}\text { SDSS name } \\
(1) \\
\end{array}$ & $\begin{array}{l}\text { RA } \\
(2) \\
\end{array}$ & $\begin{array}{c}\text { Dec } \\
(3) \\
\end{array}$ & $\begin{array}{c}z_{\text {SDSS }} \\
(4)\end{array}$ & $\begin{array}{c}u \\
(5) \\
\end{array}$ & $\begin{array}{c}g \\
(6) \\
\end{array}$ & $\begin{array}{c}r \\
(7) \\
\end{array}$ & $\begin{array}{c}i \\
(8) \\
\end{array}$ & $\begin{array}{c}z \\
(9) \\
\end{array}$ & $\begin{array}{c}J \\
(10) \\
\end{array}$ & $\begin{array}{c}H \\
(11) \\
\end{array}$ & $\begin{array}{c}K \\
(12) \\
\end{array}$ & $\begin{array}{c}E(B-V) \\
(13) \\
\end{array}$ \\
\hline $301+5210$ & 79 & 345 & 217 & . & .26 & 16.90 & 16.76 & 16.69 & 1 & 4 & 14.61 & 0.0 \\
\hline J0958+2827 & 09:58:41.21 & $+28: 27: 29.5$ & 2.120 & 20 & 18.50 & 17.84 & 17.71 & 17.51 & 16.31 & 4 & 15.41 & 0.0 \\
\hline $\mathrm{J} 1106+6400$ & :06:10.72 & $+64: 00: 09.6$ & 2.205 & 16.44 & 16.11 & 16.01 & 15.98 & 15.81 & & & & 0.0 \\
\hline $\mathrm{J} 1111+1336$ & 11:11:19.10 & $+13: 36: 03.9$ & 3.462 & 22.26 & 18.11 & 17.30 & 17.18 & 17.05 & 15.89 & 15.51 & & 0.0 \\
\hline $\mathrm{J} 1157+2724$ & $11: 57: 47.99$ & $+27: 24: 59.6$ & 2.214 & 19.98 & 19.11 & 18.25 & 17.77 & 17.31 & 16.14 & 15.43 & 14.48 & 0.2 \\
\hline $\mathrm{J} 1201+$ & $12: 01: 44.36$ & $+01: 16: 11.6$ & 3.240 & 19.50 & 17.72 & 17.39 & 17.32 & 17.27 & 15.90 & & & 0.0 \\
\hline 554 & $12: 36: 41.45$ & $+65: 54: 42.1$ & 3.358 & 20. & & & & 16 & & & & 0.0 \\
\hline 633 & $14: 21: 23.97$ & $+46: 33: 18.0$ & 3.404 & 21.96 & 17.97 & 17.38 & 17.22 & 17.09 & 16.28 & & & 0.0 \\
\hline+4417 & $14: 22: 43.02$ & $+44: 17: 21.2$ & 3.605 & 23.45 & 19.36 & 18.15 & 17.57 & 17.11 & 15.81 & & & 0.1 \\
\hline $\mathrm{J} 1521+5202$ & $15: 21: 56.48$ & $+52: 02: 38.5$ & 2.195 & 16.44 & 15.90 & 15.60 & 15.44 & 15.33 & 14 & 14 & & 0.0 \\
\hline $\mathrm{J} 1538+0855$ & $15: 38: 30.55$ & $+08: 55: 17.0$ & & & & & & & & & & 0.0 \\
\hline 50 & & -00 & & & & & & & & & & 0.0 \\
\hline J2346- & 23:46:25.66 & $-00: 16: 00.4$ & 3.489 & 21.24 & 18.69 & 17.91 & 17.75 & 17.56 & 16.87 & 16.13 & 15.30 & 0.0 \\
\hline $\mathrm{J} 0745$ & 15. & 1 & $\mathbf{r}$ & & 16.6 & 16.3 & 16 & & & & & \\
\hline $\mathrm{J} 0900+4215^{a}$ & & & & & & & & 16.58 & & & & 0.0 \\
\hline $\mathrm{J} 1201+1206^{a}$ & $12: 01: 47.90$ & $+12: 06: 30.3$ & 3.495 & 20.77 & 18.31 & 17.41 & 17.31 & 17.18 & 15.87 & 15.25 & 14.61 & 0.0 \\
\hline $\mathrm{J} 1326-0005^{a}$ & $13: 26: 54.95$ & $-00: 05: 30.1$ & 3.307 & 22.83 & 20.90 & 20.54 & 20.02 & 19.28 & 17.39 & 16.75 & & 0.3 \\
\hline $\mathrm{J} 1549+1245^{a}$ & $15: 49: 38.71$ & $+12: 45: 09.1$ & 2.386 & 20.23 & 18.67 & 17.84 & 17.38 & 16.90 & 15.86 & 14.56 & 13.52 & 0.1 \\
\hline
\end{tabular}

Notes. Columns give the following information: (1) SDSS name, (2-3) celestial coordinates, (4) redshift from SDSS DR10, (5-9) SDSS DR10 photometric data, (10-12) 2 MASS photometric data, and (13) colour excess $E(B-V)$ (Duras et al., in prep.). ${ }^{(a)}$ See Paper I for the analysis of the LBT/LUCI spectra of these objects.

Table 2. Journal of the LBT/LUCI1 observations.

\begin{tabular}{lccccc}
\hline \hline $\begin{array}{l}\text { SDSS name } \\
(1)\end{array}$ & $\begin{array}{c}\text { Obs. date } \\
(2)\end{array}$ & $\begin{array}{c}t_{\exp } \\
(3)\end{array}$ & $\begin{array}{c}\text { Grating } \\
(4)\end{array}$ & $\begin{array}{c}R \\
(5)\end{array}$ & $\begin{array}{c}\text { Seeing } \\
(6)\end{array}$ \\
\hline J0801+5210 & Feb 10th 2015 & 2400 & $150 \mathrm{~K}_{\mathrm{s}}$ & 4150 & 1.05 \\
J0958+2827 & Mar 21th 2015 & 3600 & $150 \mathrm{~K}_{\mathrm{s}}$ & 4150 & 0.75 \\
J1106+6400 & Apr 17th 2015 & 1800 & $210 \mathrm{zJHK}$ & 7838 & 0.69 \\
J1111+1336 & Apr 20th 2014 & 2400 & $150 \mathrm{~K}_{\mathrm{s}}$ & 4150 & 0.55 \\
J1157+2724 & Mar 22th 2015 & 1200 & $210 \mathrm{zJHK}^{2}$ & 7838 & 0.9 \\
J1201+0116 & Apr 27th 2015 & 1440 & $150 \mathrm{~K}_{\mathrm{s}}$ & 4150 & 0.78 \\
J1236+6554 & Apr 20th 2014 & 2400 & $150 \mathrm{~K}_{\mathrm{s}}$ & 4150 & 0.66 \\
J1421+4633 & Apr 20th 2014 & 1500 & $150 \mathrm{~K}_{\mathrm{s}}$ & 4150 & 0.52 \\
J1422+4417 & Apr 20th 2014 & 800 & $150 \mathrm{~K}_{\mathrm{s}}$ & 4150 & 0.55 \\
J1521+5202 & Apr 20th 2014 & 1200 & $210 \mathrm{zJHK}$ & 7838 & 1.01 \\
\hline
\end{tabular}

Notes. Columns give the following information: (1) SDSS name, (2) observation date, (3) exposure time (in units of s), (4) grating, (5) resolving power R, and (6) on-site average seeing (in units of arcsec).

Table 3. Journal of the SINFONI observations.

\begin{tabular}{lccccc}
\hline \hline $\begin{array}{l}\text { SDSS name } \\
(1)\end{array}$ & $\begin{array}{c}\text { Obs. date } \\
(2)\end{array}$ & $\begin{array}{c}t_{\text {exp }} \\
(3)\end{array}$ & $\begin{array}{c}\text { Grating } \\
(4)\end{array}$ & $\begin{array}{c}R \\
(5)\end{array}$ & $\begin{array}{c}\text { Spatial resolution } \\
(6)\end{array}$ \\
\hline J1538+0855 & Jul. 19th 2014 & 2400 & $\mathrm{~K}$ & 4000 & 0.3 \\
J2123-0050 & Jul 20th 2014 & 600 & $\mathrm{H}$ & 3000 & 0.2 \\
J2346-0016 & Aug18th-Sept 1st 2014 & 4800 & $\mathrm{~K}$ & 4000 & 0.3 \\
\hline
\end{tabular}

Notes. Columns give the following information: (1) SDSS name, (2) observation date, (3) exposure time (in units of s), (4) grating, (5) resolving power R, and (6) the spatial resolution obtained with AO technique (in units of arcsec).

doublet was forced to be the same as that of the $\mathrm{H} \beta$ core component. Moreover, we assumed the theoretical flux ratio for [OIII] $\lambda \lambda 4959,5007 \AA$ to be equal to $1: 3$ and the separation between [OIII] $\lambda \lambda 4959-5007 \AA$ and $\mathrm{H} \beta$ to be fixed to $98-146 \AA$ in the rest frame, respectively.
[OIII] outflow components. Specifically, one Gaussian profile with the same FWHM for each component of the [OIII] in order to accurately constrain the possible presence of subtle broad or shifted components associated with outflows. Furthermore, we left free to vary the values of the FWHM $\left(F W H M_{[\mathrm{OIII}]}^{\text {broad }}\right)$ 
Table 4. Properties of core and BLR components of the $\mathrm{H} \beta$ emission line derived from parametric model fits.

\begin{tabular}{|c|c|c|c|c|c|c|c|c|}
\hline SDSS Name & $z^{a}$ & $\begin{array}{l}F W H M_{\mathrm{H} \beta}^{\text {core }} \\
\left(\mathrm{km} \mathrm{s}^{-1}\right)\end{array}$ & $\begin{array}{l}R E W_{\mathrm{H} \beta}^{\text {core }} \\
(\AA)\end{array}$ & $\begin{array}{l}\lambda \lambda_{\mathrm{H} \beta}^{\mathrm{BLR}} \\
(\AA) \\
\end{array}$ & $\begin{array}{l}F W H M_{\mathrm{H} \beta}^{\mathrm{BLR}} \\
\left(\mathrm{km} \mathrm{s}^{-1}\right)\end{array}$ & $\begin{array}{l}R E W_{\mathrm{H} \beta}^{\mathrm{BLR}} \\
(\AA)\end{array}$ & Flux $\mathrm{H} \beta^{\mathrm{BLR}} /$ Flux $\mathrm{H} \beta^{\mathrm{Tot}}$ & Model $_{\mathrm{H} \beta}^{\mathrm{BLR}}$ \\
\hline $\mathrm{J} 0801+5210$ & $3.257 \pm 0.002$ & $1000^{b}$ & $2.4_{-0.3}^{+0.2}$ & $4862 \pm 1$ & $6080_{-160}^{+130}$ & $43.8_{-0.8}^{+0.5}$ & $0.95_{-0.09}^{+0.05}$ & Gaussian \\
\hline J0958+2827 & $3.434 \pm 0.001$ & $1000^{b}$ & $2.1_{-0.6}^{+0.2}$ & $4859 \pm 1$ & $5060_{-160}^{+140}$ & $39.4_{-0.5}^{+0.8}$ & $0.95 \pm 0.05$ & Gaussian \\
\hline $\mathrm{J} 1106+6400$ & $2.221_{-0.003}^{+0.002}$ & $1000^{b}$ & $0.6 \pm 0.2$ & $4850_{-2}^{+1}$ & $7920_{-180}^{+300}$ & $92.0 \pm 3.0$ & $0.99 \pm 0.01$ & BPL \\
\hline $\mathrm{J} 1111+1336$ & $3.490_{-0.005}^{+0.004}$ & $1000^{b}$ & $0.9_{-0.1}^{+0.3}$ & $4871_{-2}^{+1}$ & $7390 \pm 110$ & $71.0_{-1.0}^{+2.0}$ & $0.99_{-0.02}^{+0.01}$ & BPL \\
\hline $\mathrm{J} 1157+2724$ & $2.217 \pm 0.001$ & $1000^{b}$ & $3.0_{-0.4}^{+0.1}$ & $4849 \pm 1$ & $4200_{-100}^{+140}$ & $56.0 \pm 1.0$ & $0.95 \pm 0.03$ & BPL \\
\hline $\mathrm{J} 1201+0116$ & $3.248_{-0.001}^{+0.002}$ & $1000^{b}$ & $6.8_{-1.0}^{+0.3}$ & $4855 \pm 2$ & $4670_{-330}^{+130}$ & $55.0 \pm 2.0$ & $0.899_{-0.04}^{+0.05}$ & BPL \\
\hline J1236+6554 & $3.424 \pm 0.001$ & $1000^{b}$ & $1.2 \pm 0.2$ & $4848 \pm 2$ & $5330_{-220}^{+150}$ & $64.0 \pm 2.0$ & $0.98_{-0.03}^{+0.02}$ & BPL \\
\hline $\mathrm{J} 1421+4633$ & $3.454 \pm 0.002$ & $1000^{b}$ & $2.2 \pm 0.3$ & $4838 \pm 3$ & $6480 \pm 250$ & $55.0_{-1.0}^{+2.0}$ & $0.96_{-0.03}^{+0.04}$ & BPL \\
\hline $\mathrm{J} 1422+4417$ & $3.648 \pm 0.001$ & $1000^{b}$ & $3.0_{-0.4}^{+0.2}$ & $4851 \pm 2$ & $4430_{-240}^{+270}$ & $21.0 \pm 1.0$ & $0.89 \pm 0.20$ & Gaussian \\
\hline $\mathrm{J} 1521+5202$ & $2.218 \pm 0.001$ & $1000^{b}$ & $1.2 \pm 0.1$ & $4861 \pm 1$ & $7240_{-130}^{+140}$ & $25.9 \pm 0.5$ & $0.96_{-0.06}^{+0.04}$ & Gaussian \\
\hline J1538+0855 & $3.567_{-0.002}^{+0.003}$ & $1000^{b}$ & $2.1_{-0.4}^{+0.2}$ & $4843 \pm 2$ & $5490_{-210}^{+400}$ & $104.0_{-3.0}^{+4.0}$ & $0.98 \pm 0.02$ & BPL \\
\hline $\mathrm{J} 2123-0050$ & $2.282 \pm 0.001$ & $1000^{b}$ & $1.2 \pm 0.2$ & $4850 \pm 2$ & $4900+150$ & $41.1 \pm 0.5$ & $0.97_{-0.02}^{+0.01}$ & BPL \\
\hline J2346-0016 & $3.511_{-0.002}^{+0.001}$ & $1000^{b}$ & $4.2 \pm 0.9$ & $4864_{-7}^{+4}$ & $5820_{-560}^{+310}$ & $66.0_{-5.0}^{+6.0}$ & $0.94_{-0.09}^{+0.06}$ & BPL \\
\hline $\mathbf{J} 0745+4734^{c}$ & $3.225 \pm 0.001$ & $470 \pm 170$ & - & $4862 \pm 1$ & $8600_{-200}^{+230}$ & $85.5_{-1.8}^{+2.7}$ & $0.98_{-0.08}^{+0.02}$ & BPL \\
\hline $\mathrm{J}^{2900+4215^{c}}$ & $3.294 \pm 0.001$ & $300 \pm 130$ & $0.2_{-0.2}^{+1.0}$ & $4861 \pm 1$ & $3210 \pm 190$ & $54.9_{-0.5}^{+0.9}$ & $0.98 \pm 0.02$ & BPL \\
\hline $\mathbf{J 1 2 0 1 + 1 2 0 6}{ }^{c}$ & $3.512 \pm 0.002$ & $700 \pm 200$ & $10.2 \pm 1.5$ & $4865 \pm 2$ & $6160 \pm 250$ & $68.4_{-1.3}^{+1.2}$ & $0.86 \pm 0.07$ & Gaussian \\
\hline $\mathbf{J 1 3 2 6}^{-0005^{c}}$ & $3.303 \pm 0.001$ & $710 \pm 170$ & $0.4_{-0.4}^{+1.2}$ & $4862 \pm 1$ & $3700 \pm 160$ & $96.3_{-0.5}^{+0.7}$ & $0.93_{-0.09}^{+0.07}$ & BPL \\
\hline $\mathrm{J}^{1549+1245^{c}}$ & $2.365 \pm 0.001$ & $270 \pm 160$ & $0.3_{-0.3}^{+0.5}$ & $4871 \pm 2$ & $8340 \pm 280$ & $63.9_{-2.1}^{+1.8}$ & $0.98_{-0.09}^{+0.02}$ & BPL \\
\hline
\end{tabular}

Notes. Objects in boldface belong to the $[O I I I]$ sample (see Sect. 3.1 for details). ${ }^{(a)}$ Redshifts are based on the core component of $\mathrm{H} \beta$ emission line from NIR spectroscopy. ${ }^{(b)}$ Best-fit value corresponding to the maximum allowed value of FWHM (see Sect. 3.1). For these objects we only report the $F W H M_{[\mathrm{H} \beta]}^{\text {core }}$ corresponding to the best-fit values. ${ }^{\left({ }^{c}\right)}$ WISSH QSOs with LUCI1 spectra analyzed in Bischetti et al. (2017). For these objects the redshifts are based on the core component of the [OIII] emission line.

and centroids $\left(\lambda_{[\mathrm{OIII}]}^{\mathrm{broad}}\right)$ in the range $500-2500 \mathrm{~km} \mathrm{~s}^{-1}$ and $4980-$ $5034 \AA$, respectively, which are based on the values derived for these spectral parameters from the analysis of the [OIII] broad features in Paper I.

$H \beta$ broad component associated with the BLR. Specifically, one Gaussian profile with width free to vary or a broken powerlaw (BPL) component convolved with a Gaussian curve (e.g., Nagao et al. 2006, Carniani et al. 2015, Paper I).

As in Paper I, in order to fit the prominent FeII emission, we included one or two Fe II templates depending on the complexity of the iron emission. Specifically, this was done by adding to the fit one or two templates chosen through the $\chi^{2}$ minimization process among a library consisting of the three observational templates by Boroson \& Green (1992), Véron-Cetty et al. (2004), and Tsuzuki et al. (2006), and twenty synthetic FeII templates obtained with the CLOUDY plasma simulation code (Ferland et al. 2013), which takes into account different hydrogen densities, ionizing photon densities, and turbulence. The FeII template was then convolved with a Gaussian function with a velocity dispersion $\sigma$ in the range $500-4000 \mathrm{~km} \mathrm{~s}^{-1}$. The continuum was fitted by a power-law component with the normalization left free to vary and the slope derived from the best-fit to the spectral energy distribution (SED) in the wavelength range of the observations. This parametrization worked well for all but two sources, J1538+0855 and J2123-0050, for which we used a slope left free to vary. We estimated the statistical noise from the line-free continuum emission and assumed it to be constant over the entire wavelength bandwidth. Finally, the uncertainty of each parameter has been evaluated considering the variation of $\chi^{2}$ around the best fit value, with a grid step. From the corresponding $\chi^{2}$ curve, we measured the $\Delta \chi^{2}$ with respect to the minimum best-fit value. Hereafter, the reported errors refer to the one standard deviation confidence level, corresponding to $\Delta \chi^{2}=1$ for each parameter of interest.

This fit yielded a good description of the observed NIR spectrum of the WISSH QSOs. The results of our spectral analysis for the $\mathrm{H} \beta$ and [OIII] $\lambda 5007$ emission lines are listed in Table 4 and Table 5, respectively. Figure 1 shows an example of the $\mathrm{H} \beta$ and [OIII] spectral line fitting decomposition.

We estimated the spectroscopic redshift from the $\mathrm{H} \beta$ core component. The bulk of the $\mathrm{H} \beta$ emission is provided by the BLR component, which is well fitted by a broken power law for all but four cases (for J0801+5210, J0958+2827, $\mathrm{J} 1422+4417$, and $\mathrm{J} 1521+5202$ a Gaussian component was preferred). The FWHM of $\mathrm{H} \beta$ associated with the BLR has a range of $4000-8000 \mathrm{~km} \mathrm{~s}^{-1}$, with a rest-frame equivalent width of 20-100 A.

The normalization of the [OIII] emission line associated with the NLR is consistent with zero in all but three QSOs (namely J0958+2827, J1106+6400, and J1236+6554) with a $R E W_{[\mathrm{OIII}]}^{\text {core }} \approx 1 \AA$, confirming the weakness of this feature in the spectra considered here. Regarding the spectral components associated with a wind, for all but one of the QSOs $(\mathrm{J} 1538+0855)$ the best value of their FWHMs or centroids is equal to the maximum or minimum value allowed by our model (500-2500 $\mathrm{km} \mathrm{s}^{-1}$ and $4980 \AA$, respectively), or the centroids fall extremely redward to $5007 \AA$ (i.e. $>5020 \AA$ ). The combination of these findings strongly suggests that this weak $\left(R E W_{[\mathrm{OIII}]}^{\text {broad }} \leq 3 \AA\right.$ ) Gaussian component is likely to account for 
Table 5. Properties of the core (C) and broad (B) components of the $[\mathrm{OIII}] \lambda 5007$ emission line derived from parametric model fits (see Sect. 3.1).

\begin{tabular}{|c|c|c|c|c|c|}
\hline SDSS Name & Component & $\begin{array}{l}F W H M_{[\mathrm{OIII]}]} \\
\left(\mathrm{km} \mathrm{s}^{-1}\right)\end{array}$ & $\begin{array}{l}\lambda_{[\mathrm{OIII]}} \\
(\AA)\end{array}$ & $\begin{array}{l}R E W_{\text {[OIII] }} \\
(\AA)\end{array}$ & $\begin{array}{l}L_{[\mathrm{OIIII}]} \\
\left(10^{42} \mathrm{erg} \mathrm{s}^{-1}\right.\end{array}$ \\
\hline \multirow[t]{2}{*}{$\mathrm{J} 0801+5210$} & $\mathrm{C}$ & $1000^{a}$ & $5007 \pm 1$ & $<0.1$ & $<2.3$ \\
\hline & B & $1210_{-340}^{+320}$ & $5026 \pm 5$ & $0.7_{-0.3}^{+0.2}$ & $15.0 \pm 7.0$ \\
\hline \multirow[t]{2}{*}{ J0958+2827 } & $\mathrm{C}$ & $1000^{a^{-340}}$ & $5007 \pm 2$ & $1.0 \pm 0.2$ & $13.0 \pm 3.0$ \\
\hline & B & $2500^{a}$ & $4980^{a}$ & $3.5_{-0.3}^{+0.4}$ & $44.0 \pm 5.0$ \\
\hline \multirow[t]{2}{*}{$\mathrm{J} 1106+6400$} & $\mathrm{C}$ & $1000^{a}$ & $5007 \pm 4$ & $0.6 \pm 0.2$ & $10.0_{-50}^{+4.0}$ \\
\hline & B & $500^{a}$ & $5022 \pm 2$ & $0.3 \pm 0.1$ & $5.0^{+2.5 .0}$ \\
\hline \multirow[t]{2}{*}{$\mathrm{J} 1111+1336$} & $\mathrm{C}$ & $1000^{a}$ & $5007_{-2}^{+3}$ & $<0.04$ & $<0.6^{-2.0}$ \\
\hline & B & $1020_{-170}^{+200}$ & $5029^{-2} \pm 2$ & $0.9 \pm 0.2$ & $16.0 \pm 4.0$ \\
\hline \multirow[t]{2}{*}{$\mathrm{J} 1157+2724$} & $\mathrm{C}$ & $1000^{a^{-170}}$ & $5007 \pm 1$ & $<0.1$ & $<1.5$ \\
\hline & B & $500^{a}$ & $4995 \pm 2$ & $0.5 \pm 0.1$ & $6.0 \pm 2.0$ \\
\hline \multirow[t]{2}{*}{$\mathrm{J} 1201+0116$} & $\mathrm{C}$ & $1000^{a}$ & $5006 \pm 1$ & $<0.2$ & $<3.0$ \\
\hline & B & $500^{a}$ & $5022_{-35}^{+7}$ & $0.2 \pm 0.2$ & $4.7_{-47}^{+18.6}$ \\
\hline \multirow[t]{2}{*}{$\mathrm{J} 1236+6554$} & $\mathrm{C}$ & $1000^{a}$ & $5007 \pm 2$ & $1.3 \pm 0.2$ & $16.0^{-4.7} \pm 3.0$ \\
\hline & B & $820_{-210}^{+270}$ & $5022 \pm 2$ & $1.0_{-0.3}^{+0.4}$ & $12.0 \pm 4.0$ \\
\hline \multirow[t]{2}{*}{$\mathrm{J} 1421+4633$} & $\mathrm{C}$ & $1000^{-210}$ & $5007 \pm 2$ & $<0.01$ & $<1.4$ \\
\hline & B & $700 \pm 140$ & $5027 \pm 2$ & $1.4 \pm 0.2$ & $26.0 \pm 6.0$ \\
\hline \multirow[t]{2}{*}{$\mathrm{J} 1521+5202$} & $\mathrm{C}$ & $1000^{a}$ & $5007 \pm 1$ & $<0.1$ & $<0.1$ \\
\hline & B & $1270 \pm 140$ & $5033 \pm 1$ & $1.5 \pm 0.1$ & $46.0 \pm 4.0$ \\
\hline \multirow[t]{2}{*}{ J1538+0855 } & $\mathrm{C}$ & $1000^{a}$ & $5007 \pm 3$ & $<0.6$ & $<16.0$ \\
\hline & B & $1980_{-300}^{+320}$ & $4987_{-2}^{+3}$ & $7.7 \pm 0.9$ & $210.0_{-30.0}^{+40.0}$ \\
\hline \multirow[t]{2}{*}{$\mathrm{J} 2123-0050$} & $\mathrm{C}$ & $1000^{a-500}$ & $5007 \pm 2$ & $<0.01$ & $<0.3$ \\
\hline & B & 2300 & $4980^{a}$ & $2.8_{-02}^{+0.3}$ & $50.0 \pm 10$ \\
\hline \multirow[t]{2}{*}{$\mathrm{J} 2346-0016$} & $\mathrm{C}$ & $1000^{a}$ & $5007 \pm 2$ & $<0.2^{-0.2}$ & $<3.5$ \\
\hline & B & $1480 \pm 350$ & $5030_{-3}^{+4}$ & $3.2 \pm 0.5$ & $50.0 \pm 20.0$ \\
\hline \multirow[t]{2}{*}{$\mathbf{J} 0745+4734^{b}$} & $\mathrm{C}$ & $470 \pm 170$ & $5007 \pm 1$ & $3.6 \pm 0.6$ & $170 \pm 80$ \\
\hline & B & $1630 \pm 180$ & $4999 \pm 1$ & $24.8 \pm 0.8$ & $1140 \pm 290$ \\
\hline \multirow[t]{2}{*}{$\mathrm{J} 0900+4215^{b}$} & $\mathrm{C}$ & $300 \pm 130$ & $5007 \pm 1$ & $1.1 \pm 0.6$ & $50_{-30}^{+100}$ \\
\hline & B & $2240 \pm 170$ & $4999 \pm 1$ & $12.5 \pm 1.0$ & $530 \pm 100$ \\
\hline \multirow[t]{2}{*}{$\mathbf{J} 1201+\mathbf{1 2 0 6}^{b}$} & $\mathrm{C}$ & $1670 \pm 190$ & $5007 \pm 2$ & $18.8 \pm 1.1$ & $500 \pm 100$ \\
\hline & B & $940 \pm 210$ & $4990 \pm 2$ & $5.6 \pm 1.0$ & $150 \pm 80$ \\
\hline \multirow[t]{2}{*}{$\mathbf{J 1 3 2 6}-\mathbf{0 0 0 5}^{b}$} & $\mathrm{C}$ & $710 \pm 170$ & $5007 \pm 1$ & $26.5 \pm 0.6$ & $600 \pm 310$ \\
\hline & B & $1870 \pm 170$ & $4997 \pm 1$ & $50.4 \pm 0.8$ & $1140 \pm 260$ \\
\hline \multirow[t]{2}{*}{$\mathrm{J} 1549+1245^{b}$} & $\mathrm{C}$ & $270 \pm 160$ & $5007 \pm 1$ & $0.6 \pm 0.5$ & $20_{-10}^{+40}$ \\
\hline & B & $1240 \pm 210$ & $5012 \pm 1$ & $13.6 \pm 0.7$ & $470^{-10} \pm 90$ \\
\hline
\end{tabular}

Notes. Objects in boldface belong to the $[O I I I]$ sample. ${ }^{(a)}$ Best-fit value corresponding to the minimum or maximum allowed value of FWHM or centroid (see Sect. 3.1). ${ }^{(b)}$ WISSH QSOs analyzed in Paper I.

additional FeII emission not properly fitted by the templates, instead of these being due to a wind. In the case of J1538+0855, we measured a broad $\left(F W H M_{[\mathrm{OII}]}^{\text {broad }}=1980 \mathrm{~km} \mathrm{~s}^{-1}\right)$, blueshifted $\left(v_{[\mathrm{OIII}]}^{\text {broad }}=1200 \mathrm{~km} \mathrm{~s}^{-1}\right)$ component with a $R E W_{[\mathrm{OIII}]}^{\mathrm{broad}}=7.7 \AA$, mak ing us highly confident in associating this spectral feature with blueshifted $[\mathrm{OIII}]$ emission. Hereafter, we refer to the "[OIII] sample" as the group of WISSH QSOs showing a REW of the [OIII] emission line $\geq 5 \AA$, while QSOs with $R E W_{\text {[OIII] }}^{\text {broad }}<5 \AA$ are included in the "Weak $[O I I I]$ sample". Despite the fact that its NIR spectrum does not cover the [OIII] region, we also included the WISSH QSO J1422+4417 as a Weak [OIII] source, as it shows distinguishing features of this subclass (i.e. CIV velocity shift $\sim 5000 \mathrm{~km} \mathrm{~s}^{-1}$, see Sect. 3.2 and Table 6).

\subsection{Modeling of the CIV emission}

We shifted the SDSS spectra to the systemic rest frame using the redshift measured from the $\mathrm{H} \beta$ core component. The SDSS analysis was focused on the spectral region of the CIV emission line at $1549 \AA$, including spectral ranges free of emission or absorption features at both two sides of the CIV line centroid, in order to constrain the local underlying continuum. The CIV emission was fitted using a combination of up to three Gaussian profiles with spectral parameters (centroid, width, and normalization) left free to vary. We found that for all but three QSOs the best-fit model consists of a combination of two Gaussian components. For the BAL QSOs J1157+2724 and J1549+1245, for

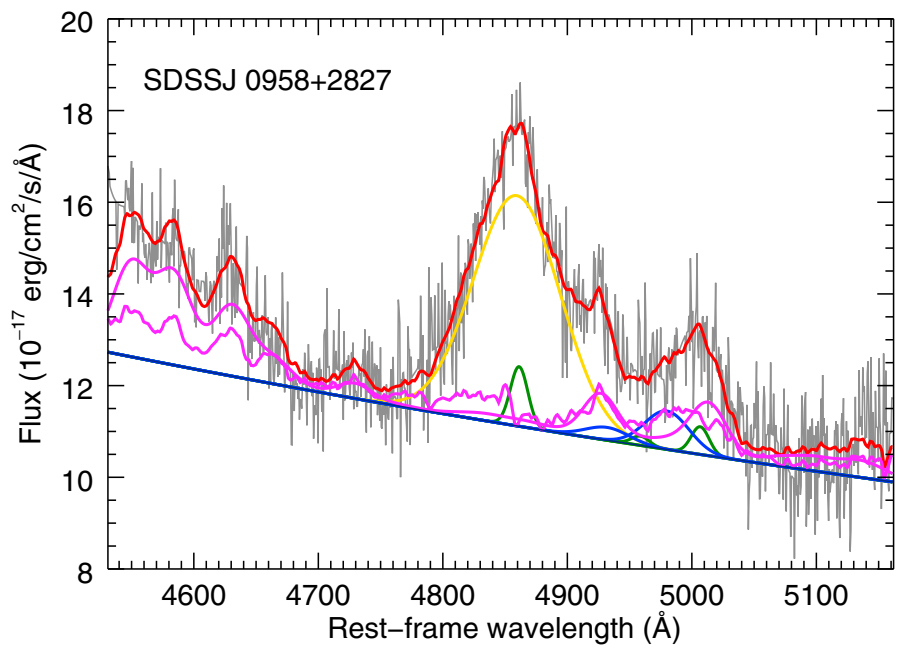

Fig. 1. Parametrization of the $\mathrm{H} \beta-[\mathrm{OIII}]$ region of the WISSH QSO J0958+2827. The red line shows the best fit to the data. Green curve refers to the core component associated with the NLR emission of $\mathrm{H} \beta$ and [OIII] emission lines. The blue curve refers to the broad blueshifted emission of [OIII] $\lambda \lambda 44959,5007 \AA$, indicative of outflow (but see Sect. 3.1 for more details). Gold curve indicates the broad component of $\mathrm{H} \beta$ associated with BLR emission. FeII emission is marked in magenta.

which the blue side of the CIV line profile is heavily affected by absorption, the CIV is modeled by one Gaussian component (see Fig. C.7 for J1157+2724 and Fig. C.16 for J1549+1245) and for the quasar $\mathrm{J} 0745+4734$, showing a very prominent peak at $1549 \AA$, the best-fit model of the CIV profile is the combination of three Gaussian components (see Fig. C.1).

We considered in our fits the presence of FeII emission by using UV FeII templates from Vestergaard \& Wilkes (2001), although FeII is not particularly strong in the CIV region. These templates were convolved with Gaussian profiles of different widths to account for the observed FeII-related emission features in the spectra. If requested, we also included in the model one or two Gaussian components to account for HeII $\lambda 1640 \AA$ and/or OIII] $\lambda 1664 \AA$ emissions.

The resulting physical parameters of the CIV emission for the 18 WISSH QSOs are listed in Table 6. We found a median value of $R E W_{\mathrm{CIV}}=21 \AA$ with the lowest value for the object $\mathrm{J} 1521+5202\left(R E W_{\mathrm{CIV}}=8 \AA\right)$, confirming its WLQ nature (Just et al. 2007). The CIV emission profile is broad with a median value of $F W H M_{\mathrm{CIV}}=7800 \mathrm{~km} \mathrm{~s}^{-1}$ and, in four cases, exceptional values of $F W H M_{\mathrm{CIV}}\left(>10000 \mathrm{~km} \mathrm{~s}^{-1}\right)$ are detected. Moreover we found that the peak of the CIV profile is blueshifted with respect to the systemic redshift, with a velocity shift ( $v_{\mathrm{CIV}}^{\text {peak }}$ hereafter) in the range of $v_{\mathrm{CIV}}^{\text {peak }}=200-7500 \mathrm{~km} \mathrm{~s}^{-1}$ in all cases except for J1326-0005, whose velocity shift is $v_{\mathrm{CIV}}^{\text {peak }}=-50 \mathrm{~km} \mathrm{~s}^{-1}$ (bearing in mind the presence of BAL features in this quasar).

The CIV emission line in the Weak [OIII] QSOs shows broad, strongly blue and asymmetric profiles, with $v_{\mathrm{CIV}}^{\text {peak }}$ in the range of $v_{\mathrm{CIV}}^{\text {peak }}=2500-7500 \mathrm{~km} \mathrm{~s}^{-1}$. This clearly indicates the presence of an outflowing component associated with this transition. According to the best-fit model, we refer to the Gaussian line with the most blueshifted centroid as the outflow component, with $v_{\text {CIV }}^{\text {out }}$ indicating its velocity shift with respect to the systemic redshift (see Fig. 2 upper panel).

The $[O I I I]$ sample exhibit the $F W H M_{\mathrm{CIV}}$ in the range of 2000-5000 $\mathrm{km} \mathrm{s}^{-1}$, and lower blueshifts than those of the 

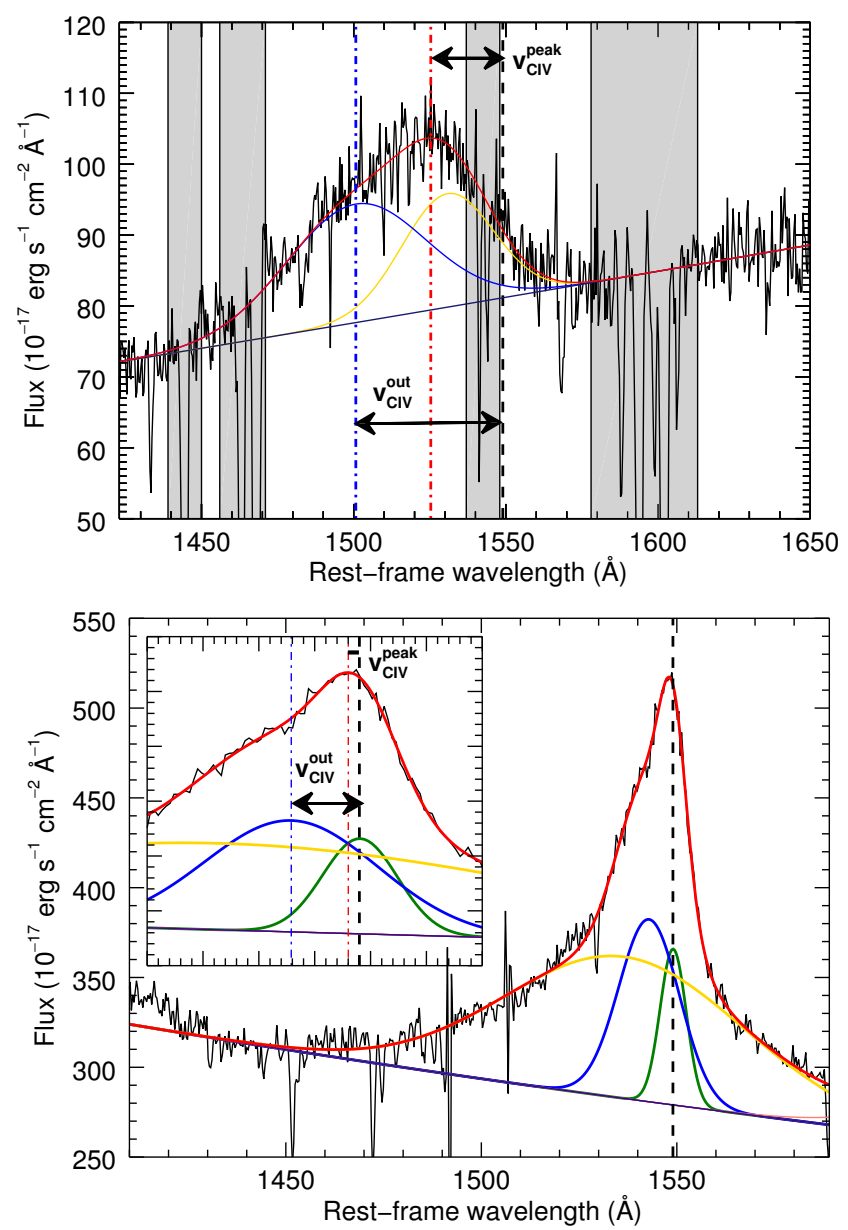

Fig. 2. Parametrization of the CIV emission line profile of the Weak $[O I I I]$ quasar $\mathrm{J} 1422+4417$ (upper panel) and the [OIII] quasar $\mathrm{J} 0745+4734$ (lower panel). In both panels the red, yellow, and blue curves refer to the best fit of the spectrum, virialized component, and outflow component of the CIV emission line, respectively. The black dashed line indicates the $\lambda_{\mathrm{CIV}}$ at $1549 \AA$. The red dotted-dashed line denotes the peak of the CIV emission line and $v_{\text {CIV }}^{\text {peak }}$ represents its velocity shift with respect to the systemic $\lambda 1549 \AA$. The blue dotted-dashed line indicates the peak of the outflow component and $v_{\mathrm{CIV}}^{\text {out }}$ is its velocity shift with respect to the systemic redshift. In the lower panel the green curve refers to an additional Gaussian component required by the fit, in order to better describe the CIV profile in this QSO.

Weak [OIII] QSOs, $v_{\mathrm{CIV}}^{\text {peak }} \leq 2000 \mathrm{~km} \mathrm{~s}^{-1}$. The profile of the CIV line in $[O I I I]$ QSOs typically is more symmetric than in Weak $[O I I I]$ objects, indicating a dominant contribution from the emission of virialized gas. We therefore consider in this case the Gaussian component with the largest FWHM as the virialized one of the CIV emission line, while the second Gaussian component with a smaller FWHM is assumed to represent the emission associated with the wind (see Fig. 2 lower panel). It is worth noting that for the vast majority of our objects we measure a virialized component with a centroid not consistent $(>2 \sigma)$ with $1549 \AA$ (see Appendix C). This can be interpreted in terms of a low-velocity component likely associated with a virialized flow in a rotating accretion disk wind (Young et al. 2007; Kashi et al. 2013). Finally, in the case of J1538+0855, an [OIII] object that also exhibits some distinctive properties of Weak [OIII] sources (see Appendix A), the virialized and outflow components of the CIV emission are assumed to be like those of Weak [OIII] sources.
Table 6. Properties of CIV $\lambda 1549$ emission line derived from parametric model fits (see Sect. 3.2).

\begin{tabular}{|c|c|c|c|c|c|}
\hline SDSS Name & $\begin{array}{c}\lambda_{\mathrm{CIV}}^{\text {peak }} \\
(\AA) \\
\end{array}$ & $\begin{array}{l}v_{\mathrm{CIV}}^{\text {peak }}, a \\
\left(\mathrm{~km} \mathrm{~s}^{-1}\right)\end{array}$ & $\begin{array}{l}R E W_{\mathrm{CIV}} \\
(\AA)\end{array}$ & $\begin{array}{l}F W H M_{\mathrm{CIV}} \\
\left(\mathrm{km} \mathrm{s}^{-1}\right)\end{array}$ & $\begin{array}{l}L_{\mathrm{CIV}} \\
\left(10^{45} \mathrm{erg} \mathrm{s}^{-1}\right)\end{array}$ \\
\hline J0745+4734 & $1548 \pm 1$ & $190 \pm 120$ & $31.0_{-4.5}^{+0.2}$ & $4320 \pm 120$ & $8.3_{-0.1}^{+0.2}$ \\
\hline $\mathrm{J} 0801+5210$ & $1535 \pm 1$ & $2720 \pm 180$ & $16.5 \pm 0.6$ & $10930_{-160}^{+120}$ & $2.9 \pm 0.1$ \\
\hline $\mathrm{J} 0900+4215$ & $1546 \pm 1$ & $560_{-110}^{+130}$ & $31.6_{-0.7}^{+0.6}$ & $4200 \pm 150$ & $6.4_{-0.2}^{+0.3}$ \\
\hline J0958+2827 & $1520 \pm 1$ & $5640 \pm 180$ & $19.0 \pm 4.0$ & $9970_{-450}^{+510}$ & $1.7 \pm 0.2$ \\
\hline $\mathrm{J} 1106+6400$ & $1534 \pm 1$ & $2870_{-170}^{+150}$ & $20.6 \pm 0.3$ & $7830 \pm 160$ & $2.8 \pm 0.1$ \\
\hline $\mathrm{J} 1111+1336$ & $1540 \pm 1$ & $1920 \pm 130$ & $20.0 \pm 1.0$ & $8210_{-190}^{+200}$ & $3.0 \pm 0.2$ \\
\hline $\mathrm{J} 1157+2724^{b}$ & $1529_{-3}^{+4}$ & $3840_{-570}^{+700}$ & $71.0_{-14.0}^{+12.0}$ & $7620_{-800}^{+650}$ & $0.9 \pm 0.1$ \\
\hline $\mathrm{J} 1201+0116$ & $1531 \pm 1$ & $3390 \pm 130$ & $17.4 \pm 0.9$ & $8460_{-250}^{+290}$ & $1.8 \pm 0.1$ \\
\hline J1201+1206 & $1544 \pm 1$ & $980_{-110}^{+130}$ & $37.0_{-2.0}^{+3.0}$ & $5110 \pm 110$ & $4.4_{-0.1}^{+0.2}$ \\
\hline $\mathrm{J} 1236+6554$ & $1532 \pm 1$ & $3360 \pm 110$ & $19.9_{-0.4}^{+0.3}$ & $7380_{-130}^{+140}$ & $2.9 \pm 0.1$ \\
\hline J1326-0005 ${ }^{b}$ & $1549 \pm 1$ & $-50_{-130}^{+180}$ & $82.0_{-7.0}^{+6.0}$ & $2110_{-230}^{+240}$ & $17.0 \pm 2.0$ \\
\hline $\mathrm{J} 1421+4633$ & $1523 \pm 1$ & $5010_{-130}^{+290}$ & $16.0 \pm 2.0$ & $10240_{-280}^{+200}$ & $2.1 \pm 0.2$ \\
\hline $\mathrm{J} 1422+4417$ & $1525 \pm 1$ & $4670 \pm 230$ & $20.0 \pm 3.0$ & $12410_{-190}^{+160}$ & $5.9 \pm 0.1$ \\
\hline $\mathrm{J} 1521+5202$ & $1511 \pm 1$ & $7420 \pm 200$ & $8.3 \pm 0.6$ & $11500_{-350}^{+370}$ & $2.1 \pm 0.1$ \\
\hline $\mathbf{J} 1538+0855$ & $1538 \pm 1$ & $2190 \pm 110$ & $20.8 \pm 0.2$ & $5160_{-30}^{+60}$ & $4.0 \pm 0.1$ \\
\hline $\mathbf{J} 1549+1245^{b}$ & $1547 \pm 1$ & $370_{-200}^{+250}$ & $23.0 \pm 1.0$ & $5720_{-210}^{+270}$ & $3.8_{-0.3}^{+0.2}$ \\
\hline $\mathrm{J} 2123-0050$ & $1537 \pm 1$ & $2330_{-150}^{+160}$ & $12.4_{-0.7}^{+0.8}$ & $6700_{-190}^{+170}$ & $1.7 \pm 0.1$ \\
\hline $\mathrm{J} 2346-0016$ & $1532 \pm 1$ & $3380+170$ & $13.0 \pm 2.0$ & $860 r_{-230}^{+140}$ & $1.2 \pm 0.1$ \\
\hline
\end{tabular}

Notes. Objects in boldface belong to the $[O I I I]$ sample. ${ }^{(a)}$ Velocity shifts of the CIV emission line are calculated from the peak of the multiple Gaussians modeling the CIV profile, with respect to the systemic redshift (see Fig. 2). ${ }^{(b)}$ Sources J1157+2724 and J1549+1245 are classified as BAL QSOs according to the Balnicity Index (BI, Weymann et al. 1991) while J1326-0005 is classified according to the modified absorption index $\left(\mathrm{AI}_{1000}>100\right.$, Bruni et al. 2012), a more conservative version of the Hall et al. (2002) AI definition. A detailed analysis of the BAL properties of the WISSH QSOs will be presented in a forthcoming paper (Bruni et al. in prep). For these objects the strong absorption in the CIV emission line affects the spectral parameters.

\section{Results}

\subsection{Properties of the [OIII] emission: $R E W_{[\mathrm{OIII}]}$ and orientation}

The observed equivalent width of the total profile of the [OIII] emission line $\left(R E W_{[\mathrm{OIII}]}\right)$ can be used as an indicator of the lineof-sight inclination as found by Risaliti et al. (2011) and Bisogni et al. (2017a), that is, $R E W_{\text {[OIII] }}=R E W_{\text {intrinsic }} / \cos (\theta)$ (with $\theta$ as the angle between the accretion disk axis and the line of sight). In these works they showed that the distribution of $R E W_{\text {[OIII] }}$ of a large sample of SDSS DR7 AGN with $0.001<\mathrm{z}<0.8$, hereafter SDSS distribution, shows a power-law tail with a slope of -3.5 at the largest $R E W_{\text {[OIII] }}$ values, which is well reproduced by assuming an isotropic [OIII] emission (which is proportional to the intrinsic disk luminosity) and a random inclination of the accretion disk with respect to the line of sight. This demonstrates that the inclination effect is likely to be responsible for the large$R E W_{\text {[OIII] }}$ power-law tail, whereby the higher the $R E W_{\text {[OIII] }}$, the higher the inclination.

The $[O I I I]$ sample of the WISSH QSOs exhibits $R E W_{\text {[OIII] }} \approx 7-80 \AA$, which is mostly due to the broad blueshifted component of the [OIII] emission. Risaliti et al. (2011) and Bisogni et al. (2017a) reported that $R E W_{\text {[OIII] }} \geq 25-30 \AA$ are associated with nearly edge-on AGN. Half of $[O I I I]$ sources populate the high tail of the SDSS $R E W_{\text {[OIII] }}$ distribution with $R E W_{\text {[OIII] }} \gtrsim 25 \AA$ (Fig. 3 top). Therefore, a high inclination, $\theta \approx 25^{\circ}-73^{\circ}$ (Bisogni et al. in prep.), can likely explain such large $R E W_{\text {[OIII] }}$.

The average value of $R E W_{\text {[OIII] }}$ for the Weak [OIII] sample is $\approx 2 \AA$. Given their high bolometric luminosities, weak [OIII] 

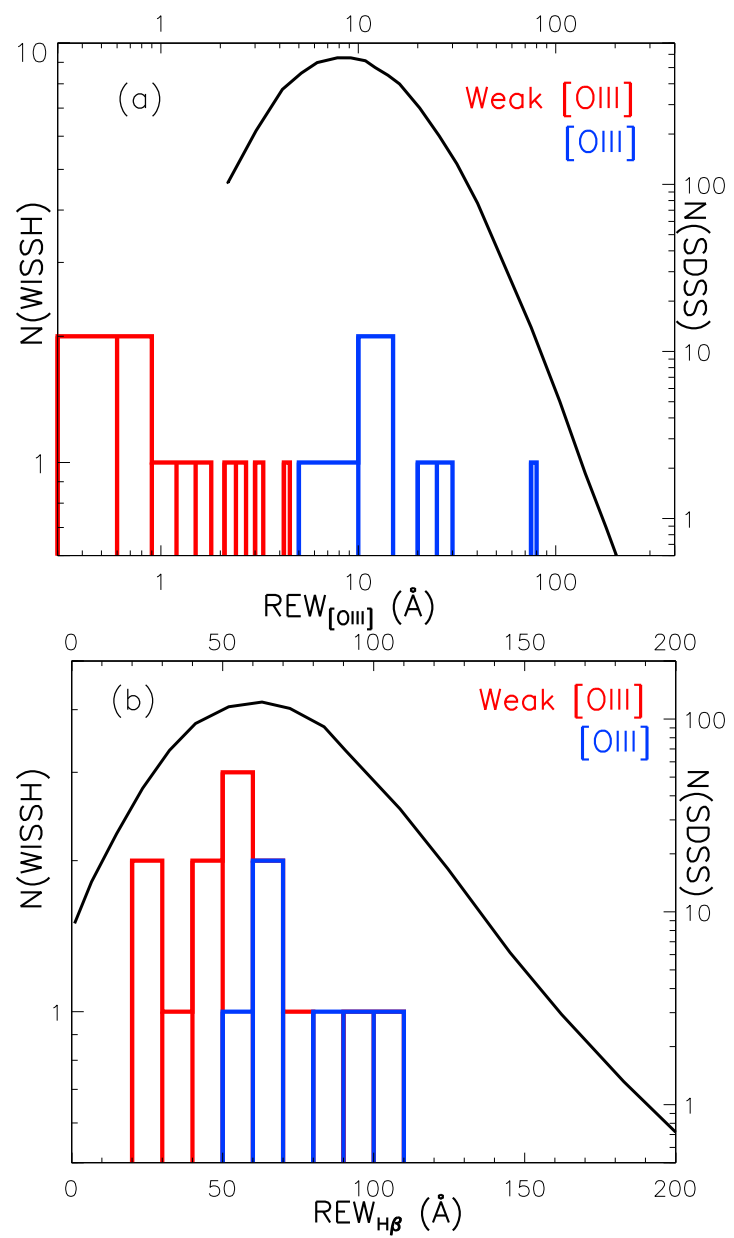

Fig. 3. Observed distribution of the WISSH QSOs for the $[O I I I]$ and Weak [OIII] samples of $(a) R E W_{[\mathrm{OIII}]}$ and $(b) R E W_{\mathrm{H} \beta}$, compared to the best fit of the $R E W_{\text {[OIII] }}$ and $R E W_{\mathrm{H} \beta}$ observed distribution of SDSS DR7 AGNs of Bisogni et al. (2017a) (black solid line).

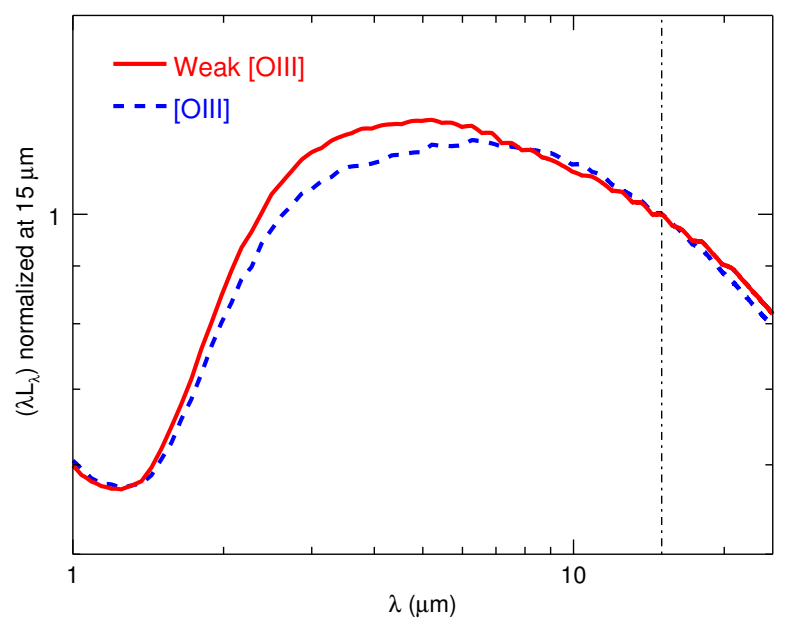

Fig. 4. Average SEDs corresponding to WISE photometry of the WISSH [OIII] (dashed blue line) and Weak [OIII] samples (red line), normalized at $15 \mu \mathrm{m}$ (dot-dashed line).

emission is expected in WISSH QSOs due to overionization of the circumnuclear gas as found in Shen \& Ho (2014) by analyzing the rest-frame optical spectra of $\sim 20000$ Type-1 SDSS QSOs. Another explanation for the reduced [OIII] emission could be linked to the ionization cone perpendicular to the

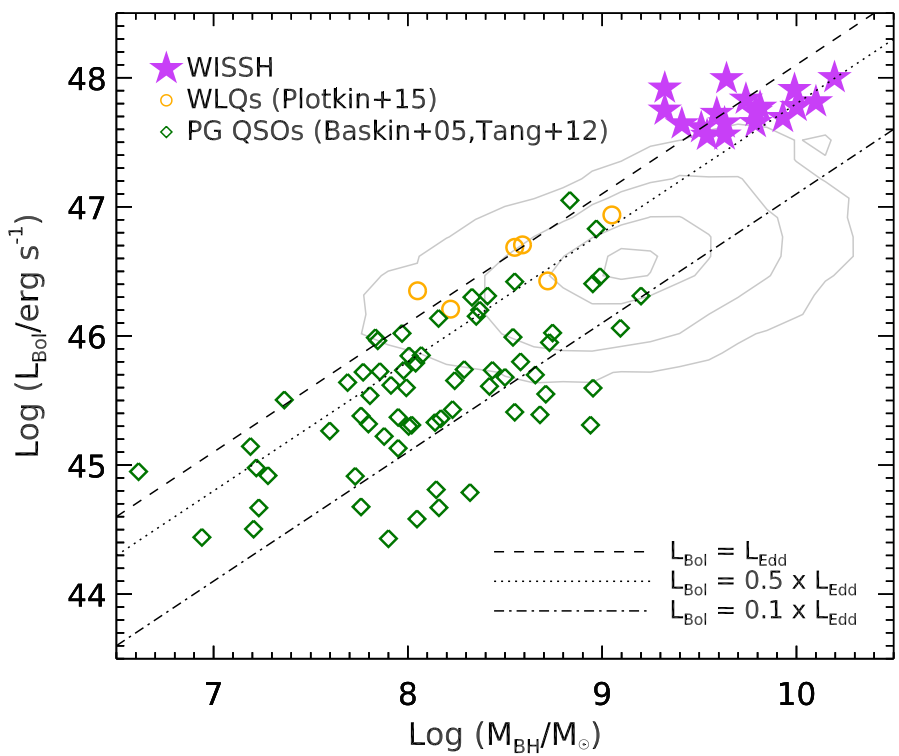

Fig. 5. Bolometric luminosity as a function of $\mathrm{BH}$ mass for the WISSH sample, compared to PG QSOs from Tang et al. (2012) and Baskin \& Laor (2005), and WLQs from Plotkin et al. (2015). Luminosity in fractions of $0.1,0.5$, and 1 Eddington luminosity are respectively indicated with dot-dashed, dotted, and dashed lines. Contours levels (0.01, 0.1, 0.5, and 0.9 relative to the peak) refer to SDSS DR7 QSOs from Shen et al. (2011).

Table 7. Logarithm of bolometric luminosity, Logarithm of intrinsic luminosity at $5100 \AA$, H $\beta$-based SMBH mass, and Eddington ratio of the WISSH QSOs.

\begin{tabular}{|c|c|c|c|c|}
\hline $\begin{array}{l}\text { SDSS Name } \\
\text { (1) }\end{array}$ & $\begin{array}{l}\log L_{\mathrm{Bol}}\left[\log L_{\mathrm{Bol}}^{\text {corr }}\right]^{a} \\
\text { (2) }\end{array}$ & $\begin{array}{l}\log L_{5100} \\
\text { (3) }\end{array}$ & $\begin{array}{l}M_{\mathrm{BH}^{b}} \\
(4)\end{array}$ & $\begin{array}{l}\lambda_{\text {Edd }} \\
(5)\end{array}$ \\
\hline J0745+4734 & $48.0[48.2]$ & 47.3 & $15.7 \pm 10.8$ & $0.5_{-0.3}^{+0.5}$ \\
\hline $\mathrm{J} 0801+5210$ & 47.8 & 47.1 & $6.2 \pm 4.3$ & $0.7 \pm 0.5$ \\
\hline J0900+4215 & $48.0[48.1]$ & 47.2 & $2.1 \pm 1.5$ & $3.1+0.6$ \\
\hline J0958+2827 & 47.6 & 46.9 & $3.5 \pm 2.4$ & $0.8^{-2.2} \pm 0.6$ \\
\hline $\mathrm{J} 1106+6400$ & 47.8 & 47.0 & $10.0 \pm 6.9$ & $0.5 \pm 0.3$ \\
\hline $\mathrm{J} 1111+1336$ & 47.7 & 47.0 & $8.5 \pm 5.9$ & $0.5 \pm 0.3$ \\
\hline $\mathrm{J} 1157+2724$ & 47.6 & 47.0 & $2.6 \pm 1.8$ & $1.3 \pm 0.9$ \\
\hline $\mathrm{J} 1201+0116$ & 47.6 & 47.0 & $3.2 \pm 2.3$ & $1.0 \pm 0.7$ \\
\hline $\mathrm{J} 1201+1206$ & $47.8[47.9]$ & 47.1 & $6.5 \pm 4.5$ & $0.7^{+0.3}$ \\
\hline $\mathrm{J} 1236+6554$ & 47.7 & 47.0 & $4.3 \pm 3.0$ & $0.8^{-0.5} \pm 0.6$ \\
\hline J1326-0005 & $47.8[48.3]$ & 47.0 & $2.1 \pm 1.6$ & $2.1_{-16}^{+0.7}$ \\
\hline $\mathrm{J} 1421+4633$ & 47.7 & 47.0 & $6.2 \pm 4.3$ & $0.6 \pm 0.4$ \\
\hline $\mathrm{J} 1422+4417$ & 48.0 & 47.3 & $4.4 \pm 3.1$ & $1.8 \pm 1.3$ \\
\hline $\mathrm{J} 1521+5202$ & 47.9 & 47.2 & $9.98 \pm 6.8$ & $0.7 \pm 0.5$ \\
\hline $\mathbf{J} 1538+0855$ & $47.8[47.8]$ & 47.1 & $5.5 \pm 3.8$ & $1.0^{+0.8}$ \\
\hline $\mathrm{J} 1549+1245$ & $47.8[47.9]$ & 47.1 & $12.6 \pm 8.7$ & $0.4^{-0.0 .9}$ \\
\hline J2123-0050 & 47.7 & 47.0 & $3.9 \pm 2.7$ & $\begin{array}{l}-0.3 \\
1.1 \pm 0.7\end{array}$ \\
\hline J2346-0016 & 47.5 & 46.8 & $4.3 \pm 3.0$ & $0.7 \pm 0.2$ \\
\hline
\end{tabular}

Notes. Objects in boldface belong to the $[O I I I]$ sample. The following information is listed: (1) SDSS name, (2) Logarithm of SED-based bolometric luminosity (in units of $\mathrm{erg} \mathrm{s}^{-1}$; Duras et al., in prep), (3) Logarithm of SED-based intrinsic luminosity at $5100 \AA$ (in units of erg s${ }^{-1}$; Duras et al., in prep), (4) $\mathrm{H} \beta$-based SMBH mass (in units of $10^{9} M_{\odot}$ ), and (5) Eddington ratio. ${ }^{(a)}$ Value of bolometric luminosity corrected for orientation effect, calculated as described in Sect. 4.2. ${ }^{(b)}$ The error associated with the $M_{\mathrm{BH}}$ includes both the statistical uncertainties and the systematic uncertainty in the virial relation itself ( $\sim 0.3 \mathrm{dex}$; see Bongiorno et al. 2014, for a complete discussion).

galaxy disk, which would intercept a lower amount of ISM, resulting in a [OIII] line with a lower REW.

Furthermore, the [OIII] WISSH QSOs typically have higher $R E W_{\mathrm{H} \beta}$ values than the Weak [OIII] sources (Fig. 3, bottom), supporting a larger inclination scenario, which leads to an observed lower underlying continuum. We also note that the 

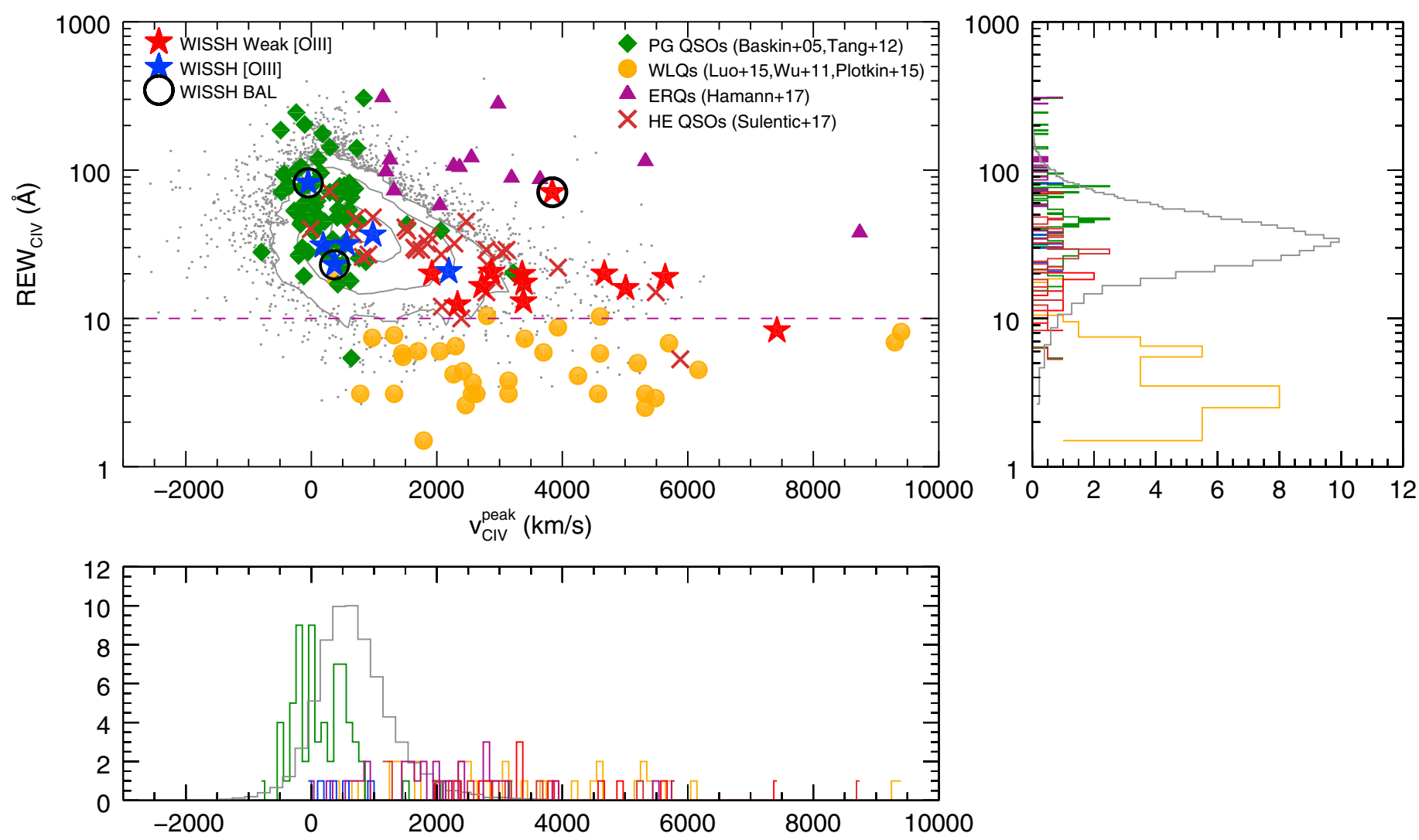

Fig. 6. $R E W_{\mathrm{CIV}}$ as a function of $v_{\mathrm{CIV}}^{\text {peak }}$ for different samples. The WISSH QSOs are indicated by blue and red stars, for the [OIII] and Weak [OIII] sample respectively. The BAL WISSH QSOs are indicated with black circles. Contours levels $(0.03,0.1,0.5$, and 0.9 relative to the peak) refer to the SDSS DR7 sample from Shen et al. (2011). The green diamonds indicate the PG QSOs from Baskin \& Laor (2005) and Tang et al. (2012). The orange circles refer to the sample of WLQs from Plotkin et al. (2015), Luo et al. (2015), and Wu et al. (2011), the brown crosses indicate the HE QSOs from Sulentic et al. (2017), the ERQs from Hamann et al. (2017) are indicated with purple triangles. The purple dashed line marks the $R E W_{\mathrm{CIV}}=10 \AA$, which is the threshold for the WLQs. The distribution of the $R E W_{\mathrm{CIV}}$ and $v_{\mathrm{CIV}}^{\text {peak }}$ are on the right and on the bottom, respectively.

$R E W_{\mathrm{H} \beta}$ distribution of the WISSH quasar is consistent with SDSS $R E W_{\mathrm{H} \beta}$ distribution. As found in Bisogni et al. (2017a), we might expect a trend of the $F W H M_{\mathrm{H} \beta}^{\mathrm{BLR}}$ also for the WISSH QSOs, that is, the width of the $\mathrm{H} \beta$ increases from low to high inclination (from small $E W_{[\mathrm{OIII}]}$ to large $E W_{[\mathrm{OIII}]}$ ). However, all the WISSH objects exhibit large values of $F W H M_{\mathrm{H} \beta}^{\mathrm{BLR}}$ and we do not observe any significant trend. This can be probably explained by the presence of ultra-massive BHs in these hyperluminous AGN (see Sect. 4.2), which heavily dilute the effect of inclination.

If the inclination plays a role in explaining the large differences in $R E W_{\text {[OIII] }}$ between [OIII] and Weak [OIII] WISSH QSOs, we also expect differences in the near- and mid-IR SED, as found by Bisogni et al. (2017b) in a large SDSS sample of QSOs. Face-on QSOs offer a direct view of the hottest dust component located in the innermost part of the torus, while in case of high-inclination sources the view of this region can be partially blocked. This is consistent with the result obtained by comparing the average SEDs corresponding to WISE photometry of the QSOs in the [OIII] and Weak [OIII] samples (Fig. 4), where sources with high $R E W_{\text {[OIII] }}$ show a flux deficit in the NIR part of the SED, around $3 \mu \mathrm{m}$ with respect to Weak [OIII] sources.

\subsection{Single epoch $H \beta$-based SMBH masses and Eddington ratios}

A common method of measuring black hole mass $\left(M_{\mathrm{BH}}\right)$ is the so-called single epoch technique (SE), which depends on
BLR size estimated from the continuum luminosity of the quasar and on the velocity dispersion of the BLR, derived from the FWHM of a specific broad emission line, that is, $\mathrm{H} \alpha, \mathrm{H} \beta, \mathrm{MgII}, \mathrm{CIV}, \mathrm{Pa} \alpha$, and $\mathrm{Pa} \beta$ depending on the redshift (McGill et al. 2008; Trakhtenbrot \& Netzer 2012; Matsuoka et al. 2013; Ricci et al. 2017). As mentioned in Sect. 3.2, the CIV emission line is affected by the presence of non-virialized components (Baskin \& Laor 2005; Richards et al. 2011), which can bias the $\mathrm{BH}$ mass estimation. The $\mathrm{H} \beta$ clouds are mostly dominated by virial motions, making this emission line the best estimator of the SMBH mass (Denney 2012). In order to estimate $M_{\mathrm{BH}}$, we used the $\mathrm{SE}$ relation for $\mathrm{H} \beta$ reported in Bongiorno et al. (2014) :

$$
\log \left(M_{\mathrm{BH}} / M_{\odot}\right)=6.7+2 \times \log \left(\frac{F W H M}{10^{3} \mathrm{~km} \mathrm{~s}^{-1}}\right)+0.5 \times \log \left(\frac{\lambda L_{\lambda}}{10^{44} \operatorname{erg~s}^{-1}}\right)
$$

For each quasar, we used the best-fit value of the FWHM of the broad component of $\mathrm{H} \beta$ line derived in Sect. 3 (see Table 4) and the continuum luminosity at $5100 \AA$. Both the $L_{5100}$ and $L_{\mathrm{Bol}}$ were obtained by fitting the UV to mid-IR photometric data (see Table 1), with Type 1 AGN SED templates of Richards et al. (2006) using a Small Magellanic Cloud extinction law (Prevot et al. 1984) with color excess $\mathrm{E}(\mathrm{B}-\mathrm{V})$ as free parameter (Duras et al. in prep.). We list the results of $\mathrm{H} \beta$-based $M_{\mathrm{BH}}$ for all the WISSH QSOs with rest-frame optical spectroscopy in Table 7.

We found that all the BHs have masses larger than $10^{9} M_{\odot}$, with nine out of 18 QSOs hosting SMBHs with 

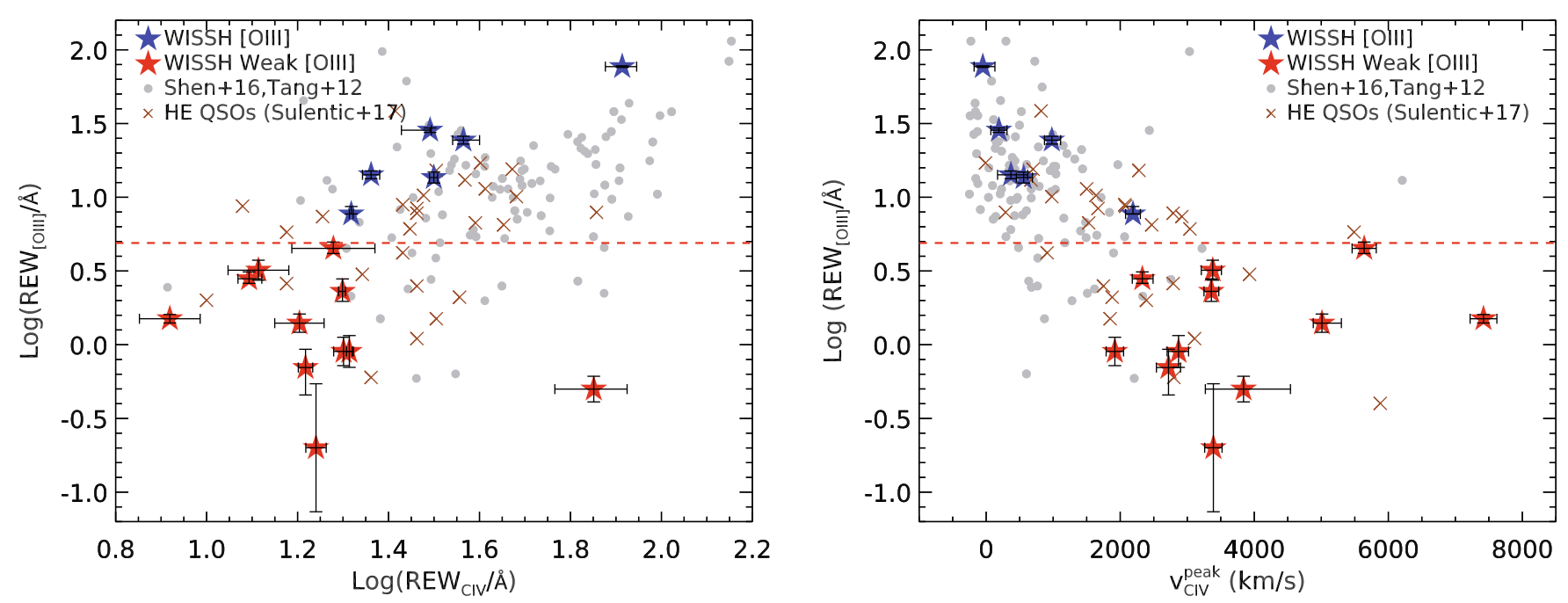

Fig. 7. Rest-frame EW of [OIII] $\lambda 5007$ as a function of $R E W_{\mathrm{CIV}}(l e f t)$ and $v_{\mathrm{CIV}}^{\text {peak }}($ right $)$ for the WISSH targets with blue stars for the [OIII] sample and red stars for the Weak [OIII] sample, compared to the samples of Shen (2016), Tang et al. (2012), and Sulentic et al. (2017, and reference therein). The dashed red line refers to the WISSH [OIII] dichotomy, with REW [OIII] boundary value of $5 \AA$.

$M_{\mathrm{BH}} \gtrsim 5 \times 10^{9} M_{\odot}$. Based on these $\mathrm{H} \beta$-based $M_{\mathrm{BH}}$ values and $L_{\mathrm{Bol}}$, we derived Eddington ratios $\lambda_{\mathrm{Edd}}=L_{\mathrm{Bol}} / L_{\mathrm{Edd}}=0.4-3.1$ (with a median value of 1 ), where $L_{\mathrm{Edd}}=1.26 \times 10^{38}\left(M_{\mathrm{BH}} / M_{\odot}\right)$ $\mathrm{erg} \mathrm{s}^{-1}$, which are also reported in Table 7. For the WISSH [OIII] objects we also estimated the bolometric luminosity $\left(L_{\mathrm{Bol}}^{\text {corr }}\right)$ corrected for the orientation effect using the mean inclination angles as determined in Bisogni et al. (in prep.). Specifically, considering the expression for the observed $R E W_{[\mathrm{OIII}]}$ distribution (Eq. (4) in Risaliti et al. 2011) as a function of the ratio between observed and intrinsic $R E W_{[\mathrm{OIII}]}$, it is possible to retrieve the inclination angle probability distribution given the observed $R E W_{\text {[OIII] }}$ and, hence, the mean values of inclination angles.

Figure 5 shows the comparison of the $M_{\mathrm{BH}}, L_{\mathrm{Bol}}$, and $\lambda_{\mathrm{Edd}}$ measured for WISSH QSOs with those derived from (i) a sample of $\sim 23000$ SDSS QSOs with $1.5 \leq z \leq 2.2$ with MgII-based $M_{\mathrm{BH}}$ (Shen et al. 2011), hereafter "SDSS sample", (ii) bright PG QSOs with $z<0.5$ with $\mathrm{H} \beta$-based $M_{\mathrm{BH}}$ (Tang et al. 2012; Baskin \& Laor 2005), and (iii) the WLQs (see Sect. 1 for the definition) with $\mathrm{H} \beta$-based $M_{\mathrm{BH}}$ from Plotkin et al. (2015). The bolometric luminosity in fractions of $0.1,0.5$, and 1 Eddington luminosity is reported. The WISSH QSOs are therefore powered by highly accreting SMBHs at the heaviest end of the $M_{\mathrm{BH}}$ function and allow us to probe the extreme AGN accretion regime and the impact of this huge radiative output on the properties of the nuclear region and the surrounding host galaxy.

\subsection{Properties of the CIV emission line}

\subsubsection{CIV velocity shift}

Previous works have found that the CIV emission line typically shows a velocity blueshift with respect to the systemic redshift (Gaskell 1982), suggesting it is associated with outflowing gas in the BLR. As already mentioned in Sect. 3.2, for the WISSH objects we measured the CIV velocity shifts $\left(v_{\mathrm{CIV}}^{\text {peak }}\right)$ from the peak of the entire CIV emission line model fit with respect to the systemic redshift (a similar approach is used in the work of Shen et al. (2011) for the "SDSS sample"). As shown in Fig. 6, we found an anti-correlation between the $v_{\mathrm{CIV}}^{\mathrm{peak}}$ and the strength of the CIV emission line for the WISSH QSOs. This result was already reported by previous studies, for example, Corbin \& Boroson (1996); Richards et al. (2002).

We compare our findings with those derived for the SDSS sample $^{2}$ (contours), PG QSOs (Tang et al. 2012; Baskin \& Laor 2005), WLQs and PHL1811-analogs (Plotkin et al. 2015; Luo et al. 2015 and Wu et al. 2011), high luminosity QSOs from Sulentic et al. (2017) identified in the Hamburg ESO survey (hereafter HE QSOs), and Extremely Red QSOs (ERQs) from Hamann et al. (2017), defined by a color (i-WISE W3) $\geq 4.6$ with a median bolometric luminosity of Log $\left(L_{\mathrm{Bol}} / \mathrm{erg} \mathrm{s}^{-1}\right) \sim 47.1 \pm 0.3$. Remarkably, Weak [OIII] WISSH QSOs show extreme velocity shifts, comparable to the largest $v_{\text {CIV }}^{\text {peak }}$ reported so far in WLQs. Furthermore, while WLQs show the distinctive property of a $R E W_{\mathrm{CIV}} \leq 10 \AA$, the WISSH sample have a $R E W_{\mathrm{CIV}} \geq 10 \AA$, demonstrating the existence of high-velocity outflows traced by high-ionization species also in sources with higher $R E W_{\mathrm{CIV}}$ than those of the WLQs. Interestingly, the ERQs show both large blueshifts and very large (i.e. $\geq 100 \AA$ ) $R E W_{\mathrm{CIV}}$, which can be interpreted in terms of a CIV emitting region with a larger covering factor with respect to the ionizing continuum than normal QSOs (Hamann et al. 2017).

In Fig. 7 the $R E W_{[\mathrm{OIII}]}$ as a function of the $R E W_{\mathrm{CIV}}$ (left) and $v_{\text {CIV }}^{\text {peak }}$ (right) is shown for the WISSH QSOs. We discovered an intriguing dichotomy between the $[O I I I]$ sample, which shows small values of $v_{\mathrm{CIV}}^{\text {peak }}\left(\leq 2000 \mathrm{~km} \mathrm{~s}^{-1}\right)$ with $R E W_{\mathrm{CIV}} \geq 20 \AA$ and the Weak [OIII] sample, exhibiting $v_{\mathrm{CIV}}^{\text {peak }} \geq 2000 \mathrm{~km} \mathrm{~s}^{-1}$ with $R E W_{\mathrm{CIV}} \leq 20 \AA$. This is also supported by the same behavior shown by the QSOs in the Shen (2016) and Tang et al. (2012) and in the HE sample ${ }^{3}$ from Sulentic et al. (2017) populating the same region of the plane $R E W_{[\mathrm{OIII}]}-v_{\mathrm{CIV}}^{\text {peak }}$. This dichotomy can be likely explained by assuming a polar geometry for the CIV winds, where the bulk of the emission is along the polar

\footnotetext{
2 We used the improved redshift estimates of Hewett \& Wild (2010), derived using emission lines not including the CIV.

3 For the HE QSOs sample we used the CIV and $\mathrm{H} \beta$ broad emission line properties listed in Sulentic et al. (2017) and the [OIII] emission lines information in Sulentic et al. $(2004,2006)$ and Marziani et al. (2009).
} 
direction, against [OIII] QSOs, which are supposed to be viewed at high inclination.

We also investigated the possible relation between the $v_{\mathrm{CIV}}^{\text {peak }}$ and the FWHM of the broad CIV emission line, by combining WISSH QSOs with other samples in Fig. 8 (top). For this purpose we used 73 SDSS QSOs at $1.5<z<3.5$ with available $\mathrm{H} \beta$ information from Shen \& Liu (2012) and Shen (2016), 19 SDSS QSOs from Coatman et al. (2016), 66 radio-quiet nonBAL PG QSOs from Baskin \& Laor (2005) and Tang et al. (2012), six WLQs from Plotkin et al. (2015) with $z \sim 1.4-1.7$, and 28 HE QSOs from Sulentic et al. (2017, and reference therein). All these objects cover a luminosity range $\log \left(L_{\mathrm{Bol}} / \mathrm{erg} \mathrm{s}^{-1}\right)=$ 44.5-48.1. Hereafter, we refer to these samples as the " $\mathrm{H} \beta$ sample". As expected, sources with large $v_{\text {CIV }}^{\text {peak }}\left(\gtrsim 2000 \mathrm{~km} \mathrm{~s}^{-1}\right)$ show a CIV emission line with a broad profile $\left(\gtrsim 6000 \mathrm{~km} \mathrm{~s}^{-1}\right)$. This suggests that for these sources the line profile is the result of the combination of a virialized component plus a strongly outflowing one. Figure 8 (bottom panel) shows the behavior of the FWHM of the outflow component of the CIV emission line resulting from our multicomponent fit (see Sect. 3.2) as a function of the $v_{\mathrm{CIV}}^{\mathrm{peak}}$. Performing the Spearman rank correlation we found $r=0.6$ and $P$-value $=5.2 \times 10^{-3}$, indicating that a large FWHM of CIV can be considered a proxy of the presence of a high velocity outflow. No clear trend is found between the $F W H M_{\mathrm{H} \beta}^{\mathrm{BLR}}$ and $v_{\mathrm{CIV}}^{\text {peak }}$, as shown in Fig. 9. Interestingly, the WISSH QSOs show $F W H M_{\mathrm{H} \beta}^{\mathrm{BLR}} \geq 4000 \mathrm{~km} \mathrm{~s}^{-1}$, even in those sources with large $v_{\mathrm{CIV}}^{\text {peak }}$, while previous works claimed the presence of large $v_{\mathrm{CIV}}^{\text {peak }}$ in QSOs with $F W H M_{\mathrm{H} \beta}^{\mathrm{BLR}}<4000 \mathrm{~km} \mathrm{~s}^{-1}$, such as low luminosity Population A QSOs (Sulentic et al. 2007; Marziani et al. 2010) and WLQs (Plotkin et al. 2015). This indicates that the inclusion of QSOs with such extreme luminosities as the WISSH ones allows us to extend the detection of large CIV blueshifts to sources with $F W H M_{\mathrm{H} \beta}^{\mathrm{BLR}}>4000 \mathrm{~km} \mathrm{~s}^{-1}$ (a similar result has been also reported for the HE sample in Sulentic et al. 2017).

\subsubsection{Mass and kinetic power of CIV winds}

We estimated the ionized gas mass $\left(M_{\text {ion }}\right)$ of the outflow associated with the outflow component of the CIV emission line for the Weak [OIII] sources according to the following formula from Marziani et al. (2016). This relation assumes a constant density scenario for the CIV emitting gas (i.e. $\mathrm{n}\left(\mathrm{C}^{3+}\right) / \mathrm{n}(\mathrm{C})=1$ ) and takes into account the carbon abundance:

$M_{\text {ion }}=9.5 \times 10^{2} \times L_{45}(C I V) \times Z_{5}^{-1} \times n_{9}^{-1}\left(M_{\odot}\right)$,

where $L_{45}(\mathrm{CIV})$ is the luminosity of the outflow component in units of $10^{45} \mathrm{erg} \mathrm{s}^{-1}, Z_{5}$ is the metallicity in units of $5 Z_{\odot}$ (i.e. the typical value measured for the BLR in high- $z$ luminous QSOs, Nagao et al. 2006), and $n_{9}$ is the gas density in units of $10^{9} \mathrm{~cm}^{-3}$. For the Weak [OIII] sample we estimated $M_{\text {ion }}=150-1100 M_{\odot}$, assuming $Z=5 Z_{\odot}$ and $n=10^{9.5} \mathrm{~cm}^{-3}$ (see Table 8). From Eq. (3) it is possible to derive the mass outflow rate $\left(\dot{M}_{\text {ion }}\right)$, that is, the mass of ionized gas passing through a sphere of radius $R$, as

$\dot{M}_{\text {ion }}=3 \times\left(M_{\text {ion }} \times v\right) / R_{1}\left(M_{\odot} \mathrm{yr}^{-1}\right)$,

where $v=v_{\mathrm{CIV}}^{\text {out }}$ and $R_{1}$ is the outflow radius in units of $1 \mathrm{pc}$. The BLR radius-luminosity relation $\left(R_{\mathrm{BLR}} \propto L_{5100}^{0.5}\right.$, e.g., Bentz et al. 2009 ) indicates a typical radius of $\sim 1 \mathrm{pc}$ for the WISSH QSOs with luminosity $L_{5100}>6 \times 10^{46} \mathrm{erg} \mathrm{s}^{-1}$. We inferred $\dot{M}_{\text {ion }}$ in

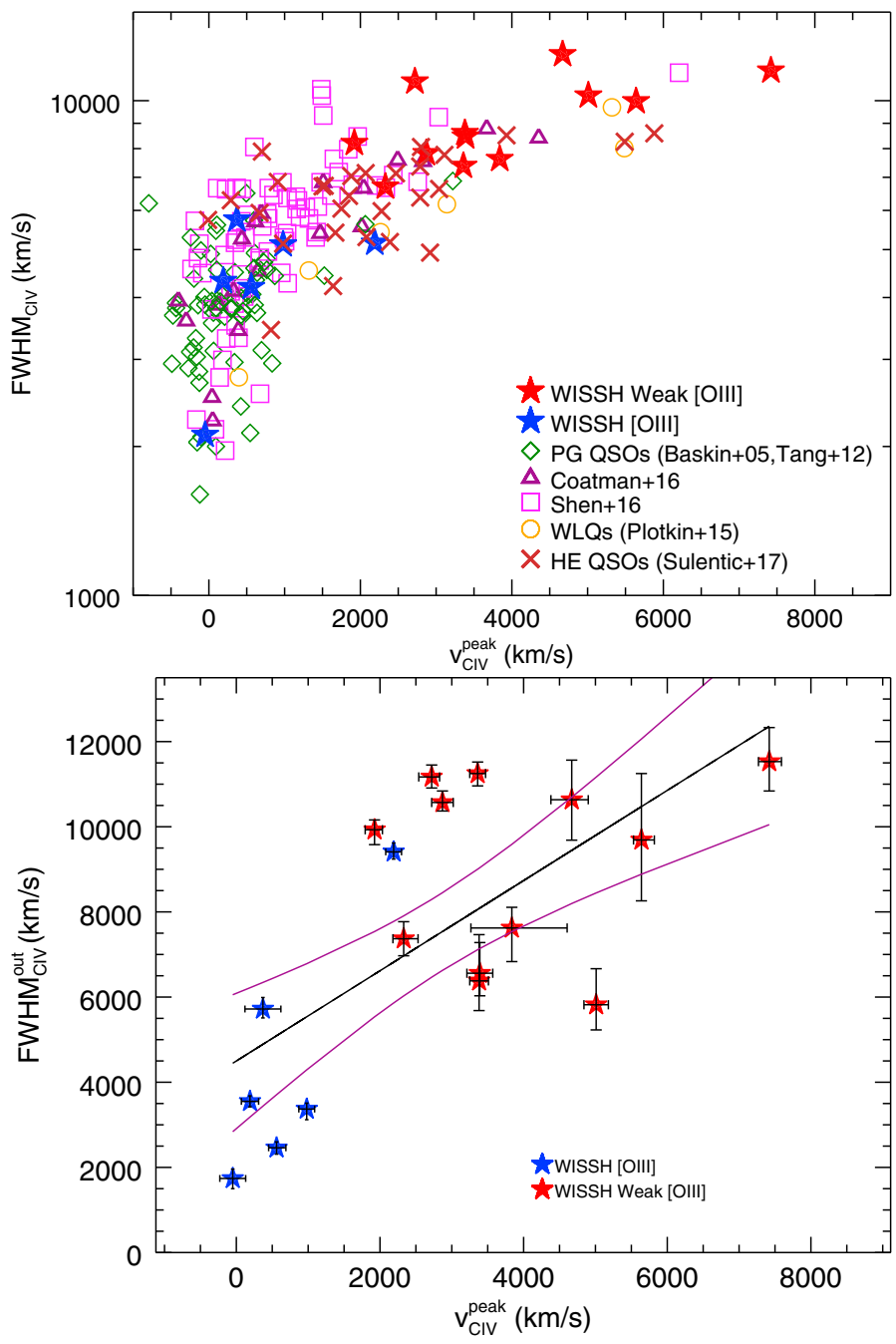

Fig. 8. $F W H M_{\mathrm{CIV}}$ of the entire emission line profile as a function of $v_{\mathrm{CIV}}^{\text {peak }}$ for the WISSH sample, compared to PG QSOs from Baskin \& Laor (2005) and Tang et al. (2012; green diamonds), WLQs from Plotkin et al. (2015; orange circles), SDSS QSOs from Shen (2016) and Coatman et al. (2016: magenta squares and purple triangles respectively), and HE QSOs from Sulentic et al. (2017; top) and FWHM(CIV) of the outflow component (bottom) as a function of $v_{\mathrm{CIV}}^{\text {peak }}$ for the WISSH sample. The black solid line indicates the best linear fits to the data and the purple dashed lines correspond to the $68 \%$ confidence interval.

the range $\approx 3-30 M_{\odot} \mathrm{yr}^{-1}$, which is similar to the accretion rate for a quasar with $L_{\mathrm{Bol}}$ corresponding to the median $L_{\mathrm{Bol}}$ of the WISSH range, $\sim 70 M_{\odot} \mathrm{yr}^{-1}$ for $L_{\mathrm{Bol}}=4 \times 10^{47} \mathrm{erg} \mathrm{s}^{-1}$, assuming a radiative efficiency of $10 \%$. Accordingly, the kinetic power of the outflow can be expressed as

$\dot{E}_{\text {kin }}=\frac{1}{2} \dot{M}_{\text {ion }} \times v^{2}\left(\operatorname{erg~s}^{-1}\right)$.

The resulting values of $\dot{E}_{\text {kin }}$ associated with the outflow component as a function of $L_{\mathrm{Bol}}$ are plotted in Fig. 10 as red filled symbols for objects in the Weak [OIII] sample.

Since the $[O I I I]$ QSOs also show an outflow component of the CIV emission (see Fig. 2), we were able to provide an estimate of $\dot{M}_{\text {ion }}, \dot{M}_{\text {ion }}$, and $\dot{E}_{\text {kin }}$ also for them. Moreover, as discussed in Sect. 4.1, [OIII] QSOs are supposed to be viewed at high inclination. Accordingly, we estimated $\dot{M}_{\text {ion }}$ and $\dot{E}_{\text {kin }}$ by assuming the so-called maximum velocity 


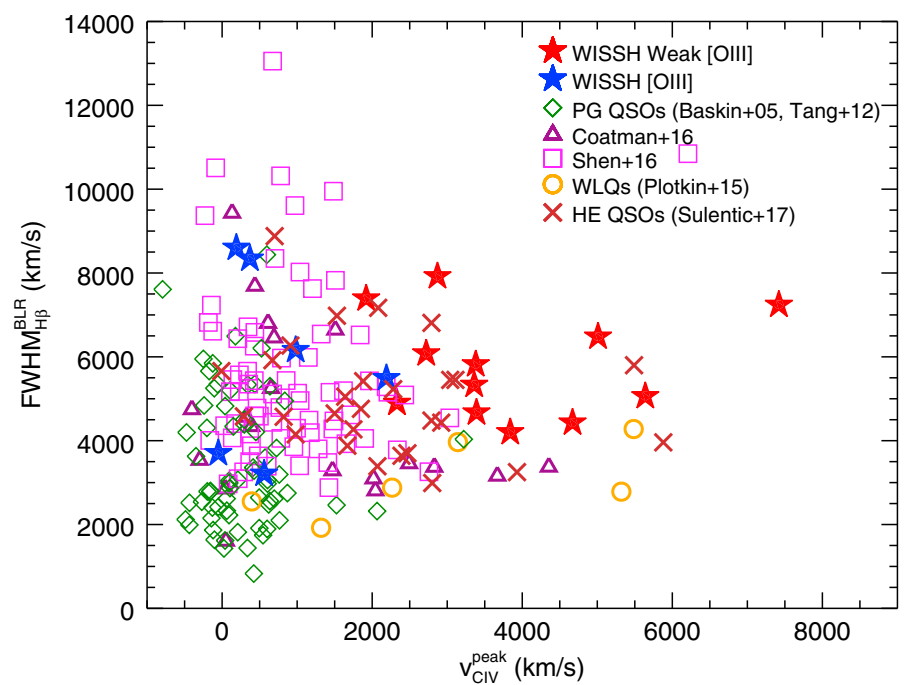

Fig. 9. $F W H M_{\mathrm{H} \beta}^{\mathrm{BLR}}$ as a function of $v_{\mathrm{CIV}}^{\text {peak }}$ for the WISSH sample, compared to PG QSOs from Baskin \& Laor (2005) and Tang et al. (2012; green diamonds), WLQs from Plotkin et al. (2015; orange circles), SDSS QSOs from Shen (2016) and Coatman et al. (2016; magenta squares and purple triangles, respectively), and HE QSOs from Sulentic et al. (2017).

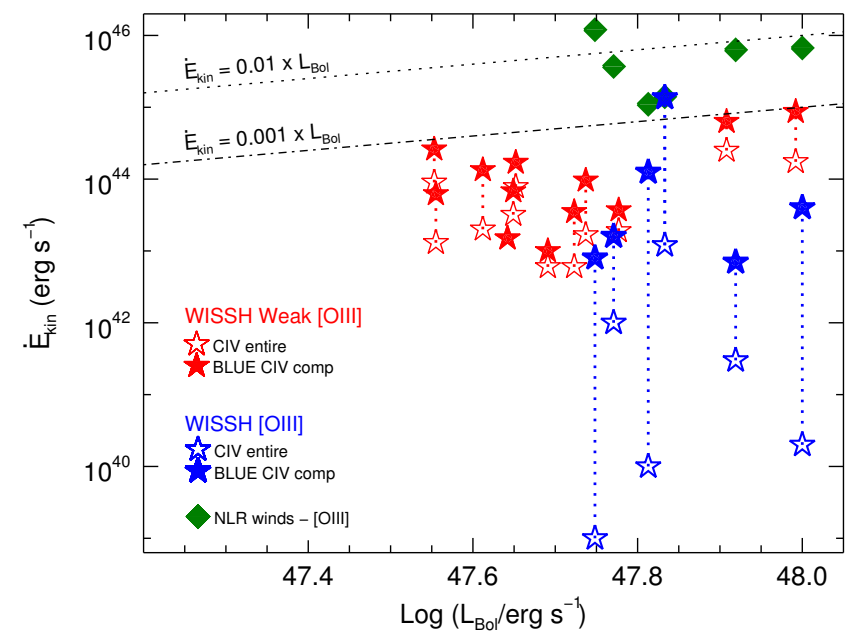

Fig. 10. Kinetic power of the BLR winds as a function of $L_{\mathrm{Bol}}$. Red filled stars refer to values of $\dot{E}_{\text {kin }}$ of the Weak [OIII] QSOs derived from the CIV outflow component using $v_{\mathrm{CIV}}^{\text {out }}$. Blue filled stars refer to values of $\dot{E}_{\text {kin }}$ of the $[O I I I]$ QSOs derived from the entire CIV profile using $v_{\mathrm{CIV}}^{\max }$. Blue and red empty stars refer to values of $\dot{E}_{\text {kin }}$ derived from the entire CIV profile using $v_{\mathrm{CIV}}^{\mathrm{peak}}$, for the [OIII] and Weak [OIII] QSOs, respectively. BLR winds are compared with WISSH NLR ionized outflows from Paper I (green diamonds).

$v_{\mathrm{CIV}}^{\max }=v_{\mathrm{CIV}}^{\text {out }}+2 \times \sigma_{\mathrm{CIV}}^{\text {out }}$, where $\sigma_{\mathrm{CIV}}^{\text {out }}$ is the velocity dispersion of the outflow component, taking into account projection effects, and can be indeed considered representative of the bulk velocity of the outflow in the case of biconically symmetric outflowing gas (see Paper I and references therein). In Fig. 10 the $\dot{E}_{\text {kin }}$ values associated with the outflow component in $[O I I I]$ sources are plotted as blue filled stars.

As mentioned in Sect. 4.3, the bulk of the virialized component of the CIV emission line is located bluewards $1549 \AA$ in our fits for both the Weak [OIII] and (even slightly) [OIII] QSOs. This means that it can be also associated with an outflow and,
Table 8. Properties of the CIV outflows derived from the outflow component of the CIV emission line.

\begin{tabular}{|c|c|c|c|c|c|}
\hline $\begin{array}{l}\text { SDSS Name } \\
\text { (1) }\end{array}$ & $\begin{array}{c}L_{\mathrm{CIV}} \\
\left(10^{45} \mathrm{erg} \mathrm{s}^{-1}\right) \\
(2)\end{array}$ & $\begin{array}{c}M_{\text {ion }} \\
\left(M_{\odot}\right) \\
(3) \\
\end{array}$ & $\begin{array}{c}v_{\mathrm{CIV}}^{\text {out } / \max , a} \\
\left(\mathrm{~km} \mathrm{~s}^{-1}\right) \\
(4)\end{array}$ & $\begin{array}{c}\dot{M}_{\text {ion }} \\
\left(M_{\odot} \mathrm{yr}^{-1}\right) \\
(5)\end{array}$ & $\begin{array}{c}\dot{E}_{\text {kin }} \\
\left(10^{42} \mathrm{erg} \mathrm{s}^{-1}\right) \\
(6) \\
\end{array}$ \\
\hline J0745+4734 & $1.90_{-0.03}^{+0.05}$ & 570 & $42000_{-130}^{+120}$ & 7.3 & 40 \\
\hline J0801+5210 & $2.6 \pm 0.1$ & 770 & $5050_{-130}^{+190}$ & 11.9 & 100 \\
\hline $\mathrm{J} 0900+4215$ & $1.4 \pm 0.1$ & 420 & $2580_{-160}^{+130}$ & 3 & 7 \\
\hline J0958+2827 & $0.7 \pm 0.2$ & 220 & $10700_{-1040}^{+1400}$ & 7 & 260 \\
\hline $\mathrm{J} 1106+6400$ & $2.3 \pm 0.1$ & 690 & $3820_{-170}^{+160}$ & 8 & 40 \\
\hline $\mathrm{J} 1111+1336$ & $2.6 \pm 0.2$ & 780 & $2360_{-170}^{+160}$ & 6 & 10 \\
\hline $\mathrm{J} 1157+2724$ & $0.9 \pm 0.1$ & 270 & $3840_{-570}^{+700}$ & 3 & 15 \\
\hline $\mathrm{J} 1201+0116$ & $0.5 \pm 0.1$ & 150 & $9830_{-250}^{+450}$ & 4 & 130 \\
\hline J1201+1206 & $1.1 \pm 0.1$ & 340 & $3640_{-220}^{+140}$ & 4 & 20 \\
\hline J1236+6554 & $2.4 \pm 0.1$ & 710 & $4620_{-130}^{+150}$ & 10 & 68 \\
\hline J1326-0005 & $6.9 \pm 0.1$ & 2090 & $1570_{-230}^{+200}$ & 10 & 8 \\
\hline $\mathrm{J} 1421+4633$ & $0.5 \pm 0.1$ & 160 & $10380_{-270}^{+460}$ & 5 & 170 \\
\hline $\mathrm{J} 1422+4417$ & $3.7 \pm 0.1$ & 1100 & $9350_{-740}^{+440}$ & 31 & 870 \\
\hline $\mathrm{J} 1521+5202$ & $1.1 \pm 0.1$ & 330 & $12570_{-680}^{+1450}$ & 13 & 630 \\
\hline J1538+0855 & $1.9 \pm 0.1$ & 590 & $13400_{-330}^{+250}$ & 24 & 1360 \\
\hline $\mathrm{J} 1549+1245$ & $3.8_{-0.3}^{+0.2}$ & 1070 & $5230_{-220}^{+300}$ & 16 & 125 \\
\hline $\mathrm{J} 2123-0050$ & $1.2 \pm 0.1$ & 350 & $4720_{-240}^{+320}$ & 5 & 35 \\
\hline J2346-0016 & $0.5 \pm 0.1$ & 150 & $7580_{-310}^{+480}$ & 3 & 62 \\
\hline
\end{tabular}

Notes. Objects in boldface belong to the $[O I I I]$ sample. The following information is listed: (1) SDSS name, (2) CIV luminosity (in units of $10^{45} \mathrm{erg} \mathrm{s}^{-1}$ ), (3) ionized gas mass (in units of $M_{\odot}$ ), (4) velocity shift of the outflow component (in units of $\mathrm{km} \mathrm{s}^{-1}$ ), (5) ionized gas mass rate (in units of $M_{\odot} / \mathrm{yr}^{-1}$ ) and (6) kinetic power (units of $10^{42} \mathrm{erg} \mathrm{s}^{-1}$ ). ${ }^{(a)} \mathrm{We}$ used $v_{\mathrm{CIV}}^{\text {out }}$ and $v_{\mathrm{CIV}}^{\max }$ in the calculation of the outflow properties of the Weak $[O I I I]$ and $[O I I I]$ QSOs respectively (see Sect. 4.3.2).

therefore, we also derived $M_{\text {ion }}, \dot{M}_{\text {ion }}$, and $\dot{E}_{\text {kin }}$ using Eqs. (3)(5), considering its luminosity in addition to that of the outflow component and adopting $v_{\mathrm{CIV}}^{\text {peak }}$ in the calculations of the outflow rates (see Table 9). The kinetic power derived by considering the entire profile of the CIV emission line are shown in Fig. 10 as red and blue empty symbols for the Weak [OIII] and [OIII] samples, respectively. In the case of Weak [OIII] sources, they are a factor of $\approx 2-10$ smaller than the values based only on the luminosity and velocity of the outflow component ( $\left.v_{\mathrm{CIV}}^{\text {peak }}<v_{\mathrm{CIV}}^{\text {out }}\right)$, due to the $\dot{E}_{\text {kin }} \propto v^{3}$ dependence. In the case of [OIII] sources, the $\dot{E}_{\text {kin }}$ calculated in this way are a factor of $\geq 100-1000$ smaller than those derived by using $v_{\mathrm{CIV}}^{\mathrm{max}}$. Accordingly, they can be considered as very conservative estimates of $\dot{E}_{\text {kin }}$.

As shown in Fig. 10, the bulk of the kinetic power of the BLR winds discovered in WISSH QSOs is $\dot{E}_{\mathrm{kin}} \sim 10^{-5} \times L_{\mathrm{Bol}}$ for both Weak $[O I I I]$ and $[O I I I]$ QSOs. It is instructive to compare these $\dot{E}_{\text {kin }}$ with those inferred for ionized NLR winds traced by [OIII]. About $20 \%$ of the BLR winds detected in WISSH QSOs show kinetic powers comparable (i.e. $10^{-3}<\dot{E}_{\mathrm{kin}} / L_{\mathrm{Bol}}<10^{-2}$ ) to those estimated for NLR winds in $[O I I I]$ sources. Remarkably, in one case, J1538+0855, the outflows associated with CIV and [OIII] have consistent $\dot{E}_{\text {kin }}$ values (see Appendix A). This would suggest that we are possibly revealing the same outflow in two different gas phases at increasing distance from the AGN (in an energy conserving scenario). This further supports the idea that this object represents a hybrid showing a mixture of the distinctive properties of the two populations of Weak [OIII] and [OIII] QSOs.

Hereafter, we consider the outflow parameters derived by using $v_{\mathrm{CIV}}^{\text {out }}$ and $v_{\mathrm{CIV}}^{\max }$ for the Weak [OIII] and the [OIII] QSOs, 
Table 9. Properties of the CIV outflows derived from the entire CIV emission line.

\begin{tabular}{|c|c|c|c|c|c|}
\hline SDSS Name & $\begin{array}{c}L_{\mathrm{CIV}} \\
\left(10^{45} \mathrm{erg} \mathrm{s}^{-1}\right) \\
(2)\end{array}$ & $\begin{array}{c}M_{\text {ion }} \\
\left(M_{\odot}\right) \\
(3)\end{array}$ & $\begin{array}{c}v_{\mathrm{CIV}}^{\text {peak }} \\
\left(\mathrm{km} \mathrm{s}^{-1}\right) \\
(4)\end{array}$ & $\begin{array}{c}\dot{M}_{\text {ion }} \\
\left(M_{\odot} \mathrm{yr}^{-1}\right) \\
(5)\end{array}$ & $\begin{array}{c}\dot{E}_{\mathrm{kin}} \\
\left(10^{42} \mathrm{erg} \mathrm{s}^{-1}\right) \\
(6)\end{array}$ \\
\hline $\mathrm{J} 0745+4734$ & $8.3_{-0.1}^{+0.2}$ & 2500 & $190 \pm 120$ & 2 & 0.02 \\
\hline $\mathrm{J} 0801+5210$ & $2.9 \pm 0.1$ & 860 & $2720 \pm 180$ & 7 & 17 \\
\hline $\mathrm{J} 0900+4215$ & $6.4_{-0.2}^{+0.3}$ & 1940 & $560_{-110}^{+130}$ & 3 & 0.3 \\
\hline J0958+2827 & $1.7 \pm 0.2$ & 520 & $5640 \pm 180$ & 9 & 90 \\
\hline $\mathrm{J} 1106+6400$ & $2.8 \pm 0.1$ & 840 & $2870_{-170}^{+150}$ & 7 & 19 \\
\hline $\mathrm{J} 1111+1336$ & $3.0 \pm 0.2$ & 900 & $1920 \pm 130$ & 5 & 6 \\
\hline $\mathrm{J} 1157+2724$ & $0.9 \pm 0.1$ & 270 & $3840_{-570}^{+700}$ & 3 & 15 \\
\hline $\mathrm{J} 1201+0116$ & $1.8 \pm 0.1$ & 540 & $3390 \pm 130$ & 6 & 20 \\
\hline J1201+1206 & $4.4_{-0.1}^{+0.2}$ & 1350 & $980_{-110}^{+130}$ & 4 & 1 \\
\hline $\mathrm{J} 1236+6554$ & $2.9 \pm 0.1$ & 870 & $3360 \pm 110$ & 9 & 32 \\
\hline $\mathbf{J} 1326-0005$ & $17 \pm 2$ & 4990 & $50_{-130}^{+180}$ & 1 & 0.001 \\
\hline $\mathrm{J} 1421+4633$ & $2.1 \pm 0.2$ & 640 & $5010_{-130}^{+290}$ & 10 & 78 \\
\hline $\mathrm{J} 1422+4417$ & $5.9 \pm 0.1$ & 1780 & $4670 \pm 230$ & 25 & 174 \\
\hline $\mathrm{J} 1521+5202$ & $2.1 \pm 0.1$ & 620 & $7420 \pm 200$ & 14 & 250 \\
\hline J1538+0855 & $4.0 \pm 0.1$ & 1210 & $2190 \pm 110$ & 8 & 12 \\
\hline $\mathrm{J} 1549+1245$ & $3.8_{-0.3}^{+0.2}$ & 1130 & $370_{-200}^{+250}$ & 1 & 0.01 \\
\hline $\mathrm{J} 2123-0050$ & $1.7 \pm 0.1$ & 520 & $2330_{-150}^{+160}$ & 4 & 6 \\
\hline J2346-0016 & $1.2 \pm 0.1$ & 350 & $3380_{-170}^{+130}$ & 4 & 13 \\
\hline
\end{tabular}

Notes. Objects in boldface belong to the $[O I I I]$ sample. The following information is listed: (1) SDSS name, (2) CIV luminosity (in units of $10^{45} \mathrm{erg} \mathrm{s}^{-1}$ ), (3) ionized gas mass (in units of $M_{\odot}$ ), (4) velocity shift (in units of $\mathrm{km} \mathrm{s}^{-1}$ ), (5) ionized gas mass rate (in units of $M_{\odot} / \mathrm{yr}^{-1}$ ), and (6) kinetic power (units of $10^{42} \mathrm{erg} \mathrm{s}^{-1}$ ).

respectively, as the most representative ones. Figure 11 (left panel) shows a comparison of $\dot{E}_{\mathrm{kin}}$ as a function of $L_{\mathrm{Bol}}$ between the NLR and BLR winds revealed in WISSH QSOs and a large collection of [OIII]-based NLR winds from a heterogeneous AGN sample reported in Fiore et al. (2017). A sizable fraction of BLR winds in Weak [OIII] and [OIII] QSOs are as powerful as NLR winds in less luminous QSOs, with $\dot{E}_{\text {kin }}<10^{43-44} \mathrm{erg} \mathrm{s}^{-1}$. This suggests that BLR winds should be taken into account to obtain a complete census of strong AGN-driven winds and comprehensively evaluate their effects of depositing energy and momentum into the ISM.

In order to give an idea of the possible uncertainties affecting the calculations of $\dot{E}_{\text {kin }}$, in Fig. 11 (left panel) we show the maximum and minimum values obtained by considering a very large range of variation for the two fundamental parameters $n_{\mathrm{e}}$ and $Z$. More specifically, the lower bound corresponds to the assumption of $n_{\mathrm{e}}=10^{10} \mathrm{~cm}^{-3}$ based on the presence of the semiforbidden line [CIII] $\lambda 1909 \AA$ (Ferland \& Elitzur 1984) and $Z=8 Z_{\odot}$ (Nagao et al. 2006), while the upper bound corresponds to the assumption of $n_{e}=10^{9} \mathrm{~cm}^{-3}$, based on the absence of forbidden lines such as [OIII] $\lambda 4363 \AA$ (Ferland \& Elitzur 1984) and $Z=3 Z_{\odot}$ (Nagao et al. 2006).

Figure 11 (right panel) displays the outflow momentum load (i.e. the ouflow momentum rate $\dot{P}_{\text {out }}\left(\equiv \dot{M}_{\text {out }} \times v_{\text {out }}\right)$ normalized to the AGN radiation momentum rate $\left.\dot{P}_{\mathrm{AGN}} \equiv L_{\mathrm{Bol}} / c\right)$ as a function of outflow velocity for different classes of outflow derived by Fiore et al. (2017), compared to those measured for the winds traced by the blueshifted CIV emission line in WISSH. The BLR winds in WISSH show velocities between those measured for X-ray ultra-fast $\left(v>10^{4} \mathrm{~km} \mathrm{~s}^{-1}\right)$ outflows (UFOs, crosses) and [OIII]-based outflows $\left(v<2000 \mathrm{~km} \mathrm{~s}^{-1}\right.$, triangles). This matches well with interpreting the outflow velocity distribution as a proxy of the distribution in radial distance from the AGN, that is, from the innermost region of the accretion disk (tens of gravitational radii for the UFOs; for example see Nardini et al. 2015; Tombesi et al. 2012, 2013; Gofford et al. 2015) up to kpc-scale in the case of the [OIII] winds (e.g., Harrison et al. 2012; Carniani et al. 2015; Cresci et al. 2015; Paper I).

The BLR winds typically exhibit a low momentum load, $\dot{P}_{\text {out }} / \dot{P}_{\text {AGN }} \lesssim 0.1$, which is a range poorly sampled by other ionized winds. Furthermore, the BLR winds seem to represent the low-power, low-velocity analogs of UFOs. The latter show $\dot{P}_{\text {out }} / \dot{P}_{\text {AGN }} \sim 1$, as expected in the case of quasi-spherical winds with electron scattering optical depth $\tau \sim 1$ produced by systems accreting at $\lambda_{\text {Edd }} \sim 1$ (e.g. King 2010). UFOs are found to have a large covering factor (Nardini et al. 2015; King \& Pounds 2015). BLR clouds in luminous AGN are expected to have a covering factor of $\sim 0.1$ (Netzer 1990). We can thus speculate that the low $\dot{P}_{\text {out }} / \dot{P}_{\mathrm{AGN}}$ of BLR winds with respect to that of UFOs may be due to both a lower $N_{\mathrm{H}}$ and a lower covering factor of the CIV outflowing gas with respect to the fast, highly ionized gas responsible for the UFOs.

\section{What is the physical driver of the CIV velocity shift?}

In order to shed light on the main physical driver of the large blueshifts of the CIV emission line observed in QSOs, we investigated the dependence of the CIV velocity shift on fundamental AGN parameters such as $L_{\mathrm{Bol}}, \lambda_{\mathrm{Edd}}$, and the UV-to-X-ray continuum slope $\left(\alpha_{\mathrm{OX}}\right)$. Previous studies indeed found that $v_{\mathrm{CIV}}^{\text {peak }}$ is correlated with all these three quantities (Marziani et al. 2016, Richards et al. 2011), but it still unclear which is the fundamental dependency. We proceeded as follows: we initially identified the main driver of $v_{\mathrm{CIV}}^{\text {peak }}$ between $L_{\mathrm{Bol}}$ and $\lambda_{\mathrm{Edd}}$ and then we studied the dependency of $v_{\mathrm{CIV}}^{\text {peak }}$ on this parameter and on $\alpha_{\mathrm{OX}}$.

\subsection{Velocity shifts versus $L_{\mathrm{Bol}}$ and $\lambda_{\mathrm{Edd}}$}

We compared the WISSH sample to QSOs for which the $\mathrm{BH}$ mass and Eddington ratio are derived from the $\mathrm{H} \beta$ emission line, the "H $\beta$ sample", because of the large uncertainties of $\mathrm{BH}$ mass estimation from the CIV emission line, affected by the nonvirialized component. All these sources therefore have reliable SMBH masses and $\lambda_{\text {Edd. }}$.

Figure 12 shows $v_{\mathrm{CIV}}^{\text {peak }}$ as a function of $L_{\mathrm{Bol}}$ (left panel, a) and $\lambda_{\text {Edd }}$ (right panel, b) for the WISSH and the "H $\beta$ sample" QSOs. The blueshifts are clearly correlated with both $L_{\mathrm{Bol}}$ and $\lambda_{\mathrm{Edd}}$. Specifically, we found a stronger correlation with $L_{\mathrm{Bol}}$ (Spearman rank $r=0.43$ and $P$-value $\left.=1.8 \times 10^{-9}\right)$ than with $\lambda_{\text {Edd }}$ (Spearman rank $r=0.33$ and $P$-value $=7.1 \times 10^{-6}$ ), bearing in mind the large scatter affecting both relations.

Moreover, performing a least-squares regression, we found that $v_{\mathrm{CIV}}^{\text {peak }} \propto L_{\mathrm{Bol}}^{0.28} \pm 0.04$. This is consistent with a radiation-driven wind scenario (Laor \& Brandt 2002), for which we indeed expect a terminal outflow velocity $\mathrm{v}_{t} \propto \sqrt{L / R_{\geq B L R}} \propto L^{0.25}$ (valid for $R \geq R_{\mathrm{BLR}}$, e.g., Netzer \& Laor 1993; Kaspi et al. 2000; Bentz et al. 2009). A similar dependence is also found for $v_{\mathrm{CIV}}^{\text {peak }}$ as a function of $M_{\mathrm{BH}}$. This lends further support to the radiative wind scenario and suggests that the radiation pressure is dominant over the Keplerian velocity field (for which a dependence of $\propto M_{\mathrm{BH}}^{0.5}$ is expected).

In order to determine which is the fundamental variable between $L_{\mathrm{Bol}}$ and $\lambda_{\mathrm{Edd}}$, we studied correlations between the residuals from (i) $\lambda_{\mathrm{Edd}}-L_{\mathrm{Bol}}$ and $v_{\mathrm{CIV}}^{\mathrm{peak}}-L_{\mathrm{Bol}}$ relations, and (ii) $L_{\mathrm{Bol}}-\lambda_{\mathrm{Edd}}$ 

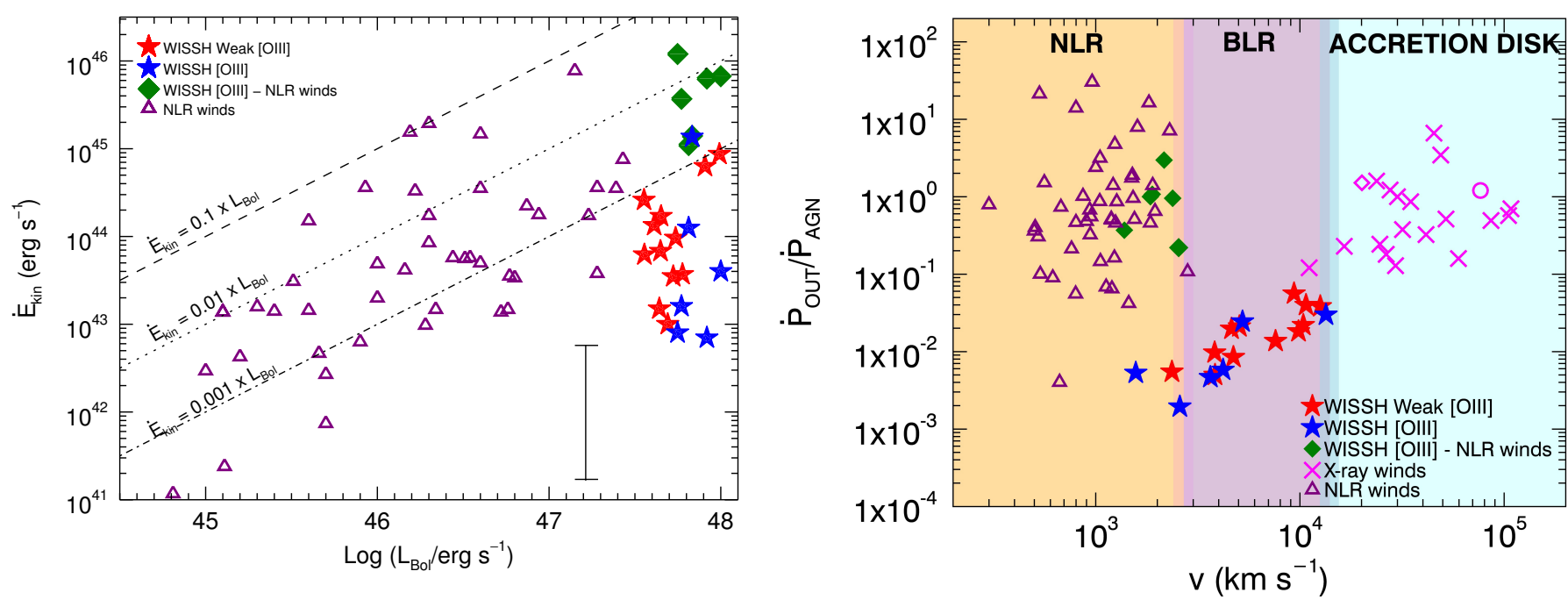

Fig. 11. Left panel: Kinetic power of the CIV outflow component as a function of $L_{\mathrm{Bol}}$ for the WISSH QSOs (red and blue stars) compared with WISSH NLR ionized outflows from Paper I (green diamonds) and other samples from literature, (e.g., Fiore et al. 2017 for details; purple triangles). The error bar (bottom right corner) is calculated as described in Sect. 4.3.2. Right panel: Wind momentum load as a function of the outflow velocity. The BLR winds traced by the CIV outflow components from the WISSH QSOs (red and blue stars) are compared with X-ray winds (magenta crosses) and ionized winds (green triangle). X-ray winds for Markarian 231 and IRASF11119+13257 are represented with a magenta diamond and circle, respectively.

and $v_{\mathrm{CIV}}^{\text {peak }}-\lambda_{\text {Edd }}$ relations (see Appendix B in Bernardi et al. 2005 for further details about residuals analysis). More specifically, we tested the hypothesis that the bolometric luminosity is the fundamental variable. In this case we expect: (i) no significant correlation between the residuals obtained from the $\lambda_{\mathrm{Edd}}-L_{\mathrm{Bol}}$ $\left(\Delta_{\lambda, \mathrm{L}}\right)$ and $v_{\mathrm{CIV}}^{\text {peak }}-L_{\mathrm{Bol}}\left(\Delta_{\mathrm{V}, \mathrm{L}}\right)$ relations; (ii) a correlation between residuals obtained from $L_{\mathrm{Bol}}-\lambda_{\mathrm{Edd}}\left(\Delta_{\mathrm{L}, \lambda}\right)$ and $v_{\mathrm{CIV}}^{\text {peak }}-\lambda_{\mathrm{Edd}}\left(\Delta_{\mathrm{V}, \lambda}\right)$ relations; (iii) that the slope of this correlation should be the same as the $v_{\mathrm{CIV}}^{\text {peak }}-L_{\mathrm{Bol}}$ relation. The $\Delta_{\lambda, \mathrm{L}}-\Delta_{\mathrm{V}, \mathrm{L}}$ residuals are plotted in Fig. 12c. In this case we derived a Spearman rank $r=$ 0.11 and $P$-value $=0.16$, which indicates no correlation between parameters as expected from (i). In Fig. $12 \mathrm{~d}$ the $\Delta_{\mathrm{L}, \lambda}$ and $\Delta_{\mathrm{v}, \lambda}$ residuals are plotted. In this case we found a strong correlation with a Spearman rank $r=0.32$ and $P$-value $=1.1 \times 10^{-5}$, with a slope consistent $(2 \sigma)$ with what we measured for the $v_{\mathrm{CIV}}^{\mathrm{peak}}-L_{\mathrm{Bol}}$ relation. We also created 1000 bootstrap samples from the residuals shown in Fig. 12d and calculated the corresponding Spearman rank, r. From the original residuals we derived $r=0.33$ with a $95 \%$ confidence interval of $0.18-0.46$, which is defined as the interval spanning the 2.5th to the 97.5th percentile of the resampled values. By combining these results, we conclude that $L_{\mathrm{Bol}}$ is the fundamental variable with respect to the $\lambda_{\mathrm{Edd}}$ and it can be considered as the main driver of the observed CIV blueshifts with respect to $\lambda_{\text {Edd }}$.

\subsection{Velocity shifts versus $L_{\mathrm{Bol}}$ and $\alpha_{\mathrm{OX}}$}

We performed the same analysis described in Sect. 5.1 considering $L_{\mathrm{Bol}}$ and $\alpha_{\mathrm{OX}}$, to investigate the primary driver of the CIV blueshifts. Figure 13 shows the $v_{\mathrm{CIV}}^{\text {peak }}$ as a function of $L_{\mathrm{Bol}}$ and $\alpha_{\mathrm{OX}}$ for 14 WISSH QSOs with both $\mathrm{H} \beta$ and X-ray measurements. For the WISSH QSOs, the $\alpha_{\text {OX }}$ was derived using the monochromatic luminosities at $2500 \AA$ obtained from broadband SED fitting and the absorption-corrected luminosities at $2 \mathrm{keV}$ (Martocchia et al. 2017). We compare our findings with those derived for 170 radio-quiet, broad-line QSOs from the Wu et al.
(2009) sample and 29 WLQs (Luo et al. 2015; Wu et al. 2011) with available UV and X-ray information.

By performing a least-square fit also for this large sample, we confirm the results reported in Sect. 5.1 about the presence of a correlation between $v_{\mathrm{CIV}}^{\text {peak }}$ and $L_{\mathrm{Bol}}$, deriving a dependence $v_{\mathrm{CIV}}^{\text {peak }} \propto$ $L_{\mathrm{Bol}}{ }^{0.25} \pm 0.06$ (Spearman rank $r=0.30$ and $P$-value $=1.1 \times 10^{-5}$; see Fig. 13a). We found a very strong anti-correlation between the $v_{\mathrm{CIV}}^{\text {peak }}$ and $\alpha_{\mathrm{OX}}$ (Fig. 13b), that is, $v_{\mathrm{CIV}}^{\text {peak }} \propto \alpha_{\mathrm{OX}}{ }^{-1.07 \pm 0.16}$ (Spearman rank $r=-0.46$ and $P$-value $=8.7 \times 10^{-13}$ ), confirming the results reported in Richards et al. (2011).

We also performed the correlations analysis between the residuals from (i) $v_{\mathrm{CIV}}^{\mathrm{peak}}-L_{\mathrm{Bol}}\left(\Delta_{\mathrm{V}, \mathrm{L}}\right)$ and $\alpha_{\mathrm{OX}}-L_{\mathrm{Bol}}\left(\Delta_{\alpha \mathrm{ox}, \mathrm{L}}\right)$ relations, and (ii) $v_{\mathrm{CIV}}^{\text {peak }}-\alpha_{\mathrm{ox}}\left(\Delta_{\mathrm{v}, \alpha \mathrm{ox}}\right)$ and $L_{\mathrm{Bol}}-\alpha_{\mathrm{ox}}\left(\Delta_{\mathrm{L}, \alpha \mathrm{ox}}\right)$ relations, based on the hypothesis that $L_{\mathrm{Bol}}$ is the fundamental parameter. However, the results reported in Fig. 13c,d are at odds with this hypothesis, indicating a clear anti-correlation between $\Delta_{\mathrm{V}, \mathrm{L}}$ and $\Delta_{\alpha \mathrm{ox}, \mathrm{L}}$, and no correlation between $\Delta_{\mathrm{V}, \alpha \mathrm{ox}}$ and $\Delta_{\mathrm{L}, \alpha \mathrm{ox}}$. Furthermore, the slope of the residuals $\Delta_{\mathrm{V}, \mathrm{L}}-\Delta_{\alpha \mathrm{ox}, \mathrm{L}}$ is consistent with that found for the $\alpha_{\mathrm{OX}}-v_{\mathrm{CIV}}^{\mathrm{peak}}$ relation. From a statistical point of view, this points to $\alpha_{\mathrm{OX}}$ as the primary driver of the blueshifts of the CIV emission line observed in these QSOs.

We note that there is a well-known strong anti-correlation between $\alpha_{\mathrm{OX}}$ and the UV luminosity ( $\sim L_{\mathrm{Bol}}$ for Type I QSOs; e.g., Vignali et al. 2003; Steffen et al. 2006; Lusso et al. 2010; Martocchia et al. 2017) according to which the steeper the $\alpha_{\mathrm{OX}}$, the higher the luminosity. Therefore, both selecting steep $\alpha_{\mathrm{OX}}$ or high $L_{\mathrm{Bol}}$ allows us to pick up fast outflows. We can conclude that the strength and the slope of the ionizing continuum is the main driver of the BLR winds. In Table 10 a summary of correlations of the CIV velocity shifts with physical quantities such as $L_{\mathrm{Bol}}, \lambda_{\mathrm{Edd}}, M_{\mathrm{BH}}$ and $\alpha_{\mathrm{OX}}$ is reported. Results from residuals correlations are also listed.

The dependence of the velocity shift on both $L_{\mathrm{Bol}}$ and $\alpha_{\mathrm{OX}}$ is in agreement with our scenario of radiation-driven wind, according to which a strong UV continuum is necessary to launch the wind but the level of extreme UV (EUV) and X-ray emission (i.e., up to $2 \mathrm{keV}$ ) is crucial to determine its existence, 

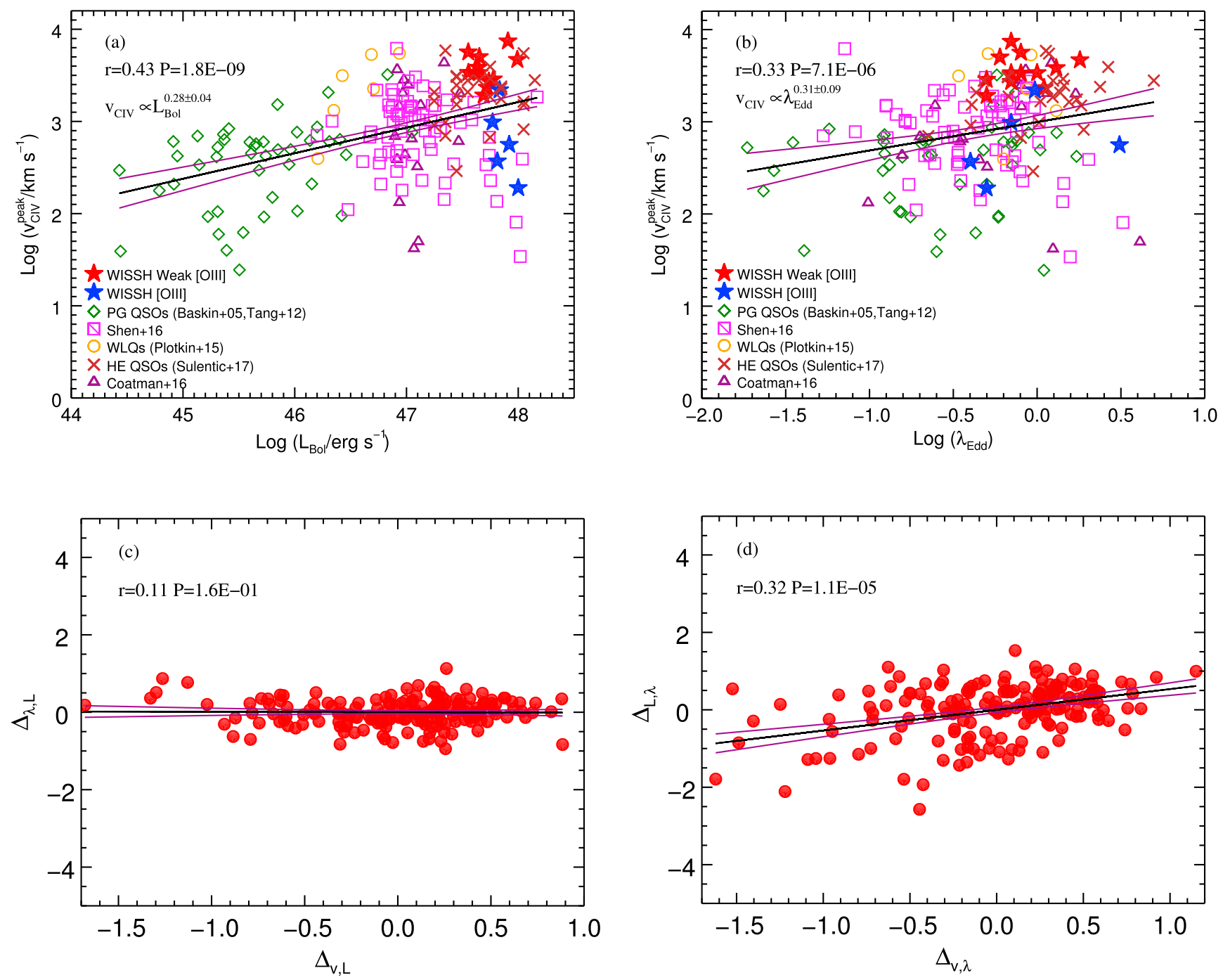

Fig. 12. The velocity shift $v_{\mathrm{CIV}}^{\text {peak }}$ as a function of $L_{\mathrm{Bol}}(a)$ and as a function of $\lambda_{\text {Edd }}(b)$, for the WISSH sample (blue and red stars) compared to the PG QSOs from Baskin \& Laor (2005) and Tang et al. (2012; green diamonds), WLQs from Plotkin et al. (2015; orange circles), SDSS QSOs from Shen (2016) and Coatman et al. (2016; magenta squares and purple triangles), and HE QSOs from Sulentic et al. (2017, and reference therein). The $L_{\mathrm{Bol}}$ for the HE sample has been derived from $L_{5100}$ applying a bolometric correction of 5.6, as suggested by Runnoe et al. (2012) for the luminosity range of the HE sample. $c$ ) Residuals plot of $\lambda_{\mathrm{Edd}}-L_{\mathrm{Bol}}$ and $v_{\mathrm{CIV}}^{\text {peak }}-L_{\mathrm{Bol}}$ relations. $d$ ) Residuals plot of $L_{\mathrm{Bol}}-\lambda_{\mathrm{Edd}}$ and $v_{\mathrm{CIV}}^{\text {peak }}-\lambda_{\mathrm{Edd}}$ relations. The black lines indicate the best linear fits to the data and the purple lines correspond to the $68 \%$ confidence interval.

since strong X-ray radiation can easily overionize the gas and hamper an efficient line-driving mechanism. On the contrary, an X-ray weaker emission with respect to the optical-UV accretion disk emission can allow UV line opacity (Leighly 2004; Richards et al. 2011). Furthermore, as suggested by Wu et al. (2009), the increasing steepness of the $\alpha_{\text {OX }}$ (i.e., the softening of EUV-X-ray emission) at progressively higher UV luminosities may also explain the weakness of the CIV emission line (ionization potential $64.45 \mathrm{eV}$ ) in luminous QSOs; the strength of the line indeed depends on the number of the photons available to produce it, whereby a deficit of such ionizing photons leads to CIV weaker emission lines, as typically observed in Weak [OIII] QSOs and WLQs.

\section{Summary and conclusions}

We have presented the results from the analysis of thirteen sources in the WISSH quasar sample with NIR spectroscopy from LBT/LUCI or VLT/SINFONI observations, in addition to five sources discussed in Paper I. We stress that these objects have been selected on the basis of a redshift for which the LBT/LUCI spectrum covers the [OIII] wavelength range and, therefore, can be considered as randomly selected from the entire WISSH QSOs sample. Our analysis has been performed with the goal of deriving the properties of (i) $\mathrm{H} \beta$ and [OIII] emission lines from the optical rest-frame spectra, and (ii) the CIV emission line by using rest-frame UV SDSS spectra. Our main findings can be summarized as follows:

- All the 18 WISSH QSOs considered here exhibit SMBHs with mass larger than $10^{9} M_{\odot}$, with $50 \%$ of them hosting very massive SMBHs with $M_{\mathrm{BH}} \gtrsim 5 \times 10^{9} M_{\odot}$. Based on these $\mathrm{H} \beta$-based $M_{\mathrm{BH}}$ values, we derived Eddington ratios $0.4<\lambda_{\text {Edd }}<3.1$. This supports the evidence that the WISSH QSOs are powered by highly accreting SMBHs at the massive end of the SMBH mass function.

- According to the $R E W_{\text {[OIII] }} \geq$ or $<5 \AA$, the WISSH QSOs can be divided into two samples: [OIII] and Weak [OIII] samples, respectively. In particular, six sources exhibit 

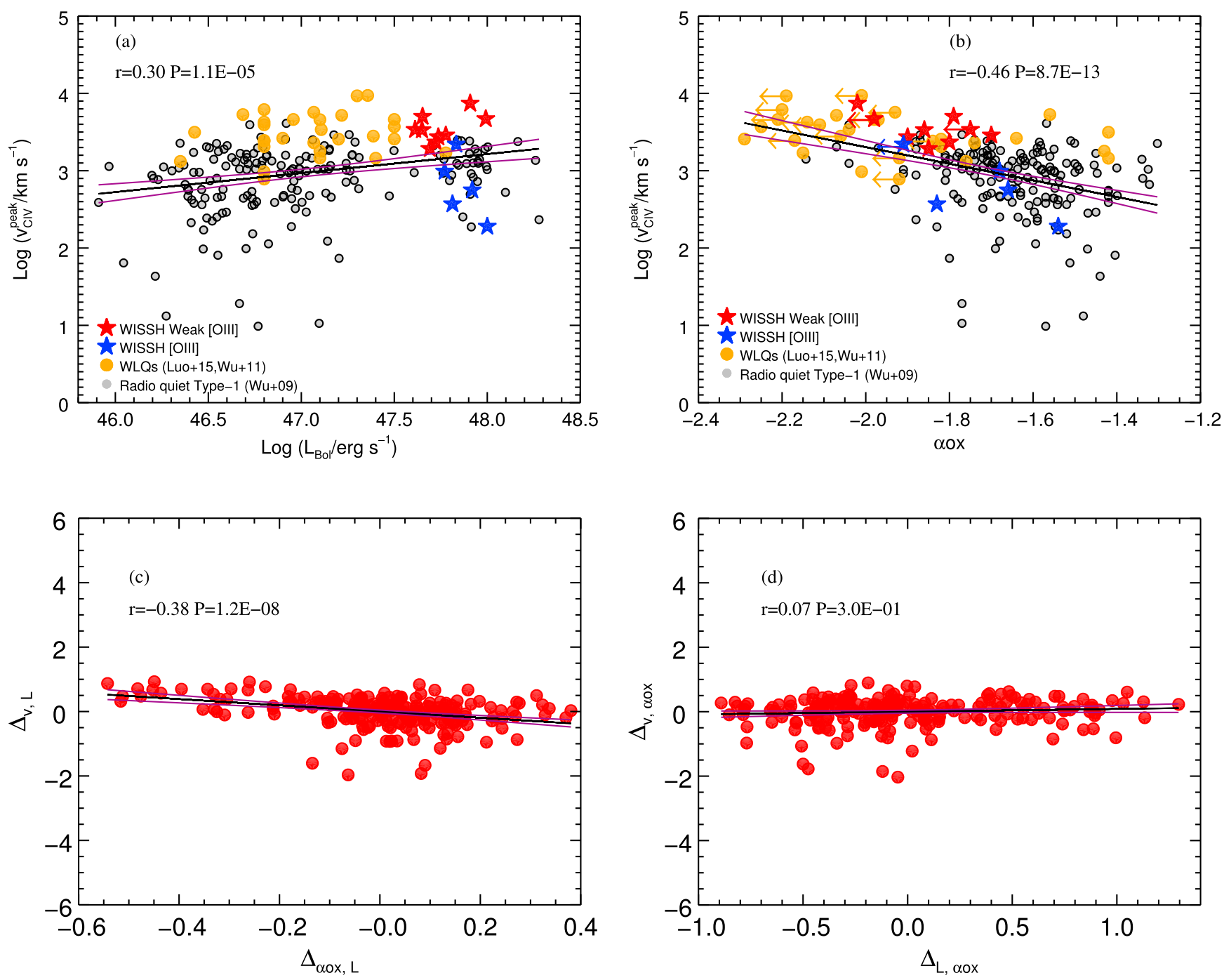

Fig. 13. The velocity shift $v_{\mathrm{CIV}}^{\text {peak }}$ as a function of $L_{\mathrm{Bol}}(a)$ and $\alpha_{\mathrm{OX}}(b)$, for the WISSH sample (blue and red stars) compared to radio quiet type-1 QSOs from Wu et al. (2009; black circles) and WLQs from Wu et al. (2011) and Luo et al. (2015; orange circles). $c$ ) Residuals plot of $v_{\mathrm{CIV}}^{\text {peak }}-L_{\mathrm{Bol}}$ and $\alpha_{\mathrm{OX}}-L_{\mathrm{Bol}}$ relations. $d$ ) Residuals plot of $v_{\mathrm{CIV}}^{\text {peak }}-\alpha_{\mathrm{OX}}$ and $L_{\mathrm{Bol}}-\alpha_{\mathrm{OX}}$ relations. The black lines represent the best linear fits to the data and the purple lines correspond to the $68 \%$ confidence interval.

$R E W_{\text {[OII] }]} \approx 7-70 \AA$, showing [OIII] profiles dominated by a broad blueshifted component, and 11 sources have $R E W_{[\mathrm{OIII}]} \approx$ $0.3-3 \AA$.

- As reported in Sect. 4.1, there is more than one explanation to ascribe to the WISSH $R E W_{\text {[OIII] }}$ distribution. One is in terms of orientation effect, with the [OIII] sample likely being seen at high ( $\approx 25-73 \mathrm{deg}$ ) inclination. This leads to a lower continuum luminosity and, hence, high $R E W_{\text {[OIII] }}$ values. On the contrary, the Weak [OIII] sources (including J1422+4417, see Sect. 3.1) are likely associated with nearly face-on AGNs. However, the weak [OIII] emission for the WISSH QSOs is expected to be due to the high bolometric luminosities of these sources. Indeed the [OIII] emission tends to decrease because of the over-ionization from the central engine, as found by Shen \& Ho (2014). A further explanation for the difference in $R E W_{\text {[OIII] }}$ between the [OIII] and Weak $[O I I I]$ may be ascribed to the presence of a kpc-scale ionization cone oriented along the galaxy disk for the [OIII] objects, leading to a larger amount of gas to be ionized, while an ionization cone oriented perpendicular to the galaxy disk in the case of Weak [OIII] sample leads to a lower content of [OIII] gas to be ionized.
- Most of the WISSH QSOs exhibits $10 \lesssim R E W_{\mathrm{CIV}} \lesssim 20 \AA$ with the peak of the CIV emission line profile blueshifted with respect to the systemic redshift $\left(v_{\mathrm{CIV}}^{\text {peak }} \sim 2000-8000 \mathrm{~km} \mathrm{~s}^{-1}\right)$, indicating that the emitting gas is outflowing. This suggests that the luminosity-based selection criterion of WISSH is very effective in collecting strong CIV winds. Historically, such large CIV blueshifts have been associated with fainter CIV emitting QSOs, that is, the WLQs (Plotkin et al. 2015), although very recently Hamann et al. (2017) have reported the existence of ERQs showing CIV winds and extremely large $R E W_{\mathrm{CIV}}$ (i.e., $>100 \AA$ ). We found that QSOs belonging to the Weak [OIII] sample, representing $70 \%$ of the WISSH sample analyzed here, show a broad asymmetric blueward and relatively weak (i.e., $R E W_{\mathrm{CIV}}<$ $20 \AA$ ) CIV line profile (see Fig. 7), with the peak of the entire CIV profile extremely blueshifted ( $v_{\mathrm{CIV}}^{\text {peak }} \geq 2000 \mathrm{~km} \mathrm{~s}^{-1}$ up to $8000 \mathrm{~km} \mathrm{~s}^{-1}$ ). On the contrary, $30 \%$ of the WISSH QSOs (i.e., [OIII] sample) have a peaked CIV profile with $R E W_{\mathrm{CIV}}>$ $20 \AA$ and $v_{\mathrm{CIV}}^{\text {peak }} \leq 2000 \mathrm{~km} \mathrm{~s}^{-1}$. High-luminosity, opticallyselected HE QSOs from Sulentic et al. (2017) follow a similar trend by populating the same region of the WISSH QSOs in the 
Table 10. Correlations of $v_{\mathrm{CIV}}^{\text {peak }}$ with AGN fundamental parameters.

\begin{tabular}{lccc}
\hline \hline $\begin{array}{l}\text { Correlation } \\
(1)\end{array}$ & $\begin{array}{c}\text { Slope } \\
(2)\end{array}$ & $\begin{array}{c}r \\
(3)\end{array}$ & $\begin{array}{c}P \text {-value } \\
(4)\end{array}$ \\
\hline$v_{\mathrm{CIV}}^{\text {peak }}$ vs. $L_{\mathrm{Bol}}{ }^{a}$ & $0.28 \pm 0.04$ & 0.43 & $1.8 \times 10^{-9}$ \\
$v_{\text {peak }}^{\text {peak }}$ vs. $\lambda_{\mathrm{Edd}}{ }^{a}$ & $0.31 \pm 0.09$ & 0.33 & $7.1 \times 10^{-6}$ \\
${ }_{\text {ClV }}^{\text {peak }}$ vs. $M_{\mathrm{BH}}{ }^{a}$ & $0.30 \pm 0.05$ & 0.34 & $3.5 \times 10^{-6}$ \\
$\Delta_{\lambda, \mathrm{L}}$ vs. $\Delta_{\mathrm{V}, \mathrm{L}}{ }^{a}$ & $-0.01 \pm 0.06$ & 0.11 & $1.6 \times 10^{-1}$ \\
$\Delta_{\mathrm{L}, \lambda}$ vs. $\Delta_{\mathrm{V}, \lambda}{ }^{a}$ & $0.54 \pm 0.10$ & 0.32 & $1.1 \times 10^{-5}$ \\
$v_{\mathrm{Cek}}^{\text {peak }}$ vs. $L_{\mathrm{Bol}}{ }^{b}$ & $0.25 \pm 0.06$ & 0.30 & $1.1 \times 10^{-5}$ \\
$v_{\mathrm{CIV}}^{\text {peak }}$ vs. $\alpha_{\mathrm{OX}}{ }^{b}$ & $-1.07 \pm 0.16$ & -0.46 & $8.7 \times 10^{-13}$ \\
$\Delta_{\mathrm{V}, \mathrm{L}}$ vs. $\Delta_{\alpha \mathrm{ox}, \mathrm{L}}{ }^{b}$ & $-0.97 \pm 0.18$ & -0.38 & $1.2 \times 10^{-8}$ \\
$\Delta_{\mathrm{V}, \alpha \mathrm{ox}}$ vs. $\Delta_{\mathrm{L}, \alpha \mathrm{ox}}{ }^{b}$ & $0.09 \pm 0.06$ & 0.07 & $3.0 \times 10^{-1}$ \\
\hline
\end{tabular}

Notes. The following information is listed. (1) Correlations of $v_{\mathrm{CIV}}^{\text {peak }}$ with $L_{\mathrm{Bol}}, \lambda_{\mathrm{Edd}}, M_{\mathrm{BH}}$, and $\alpha_{\mathrm{OX}}$, and between residuals (see Sect. 5.1 and 5.2), (2) least-square regression slope, (3) Spearman rank and (4) null hypothesis probability. ${ }^{(a)}$ Correlations derived for the WISSH QSOs and other QSO samples from literature with $\mathrm{H} \beta$ measurements, as detailed in Sect. 5.1. ${ }^{(b)}$ Correlations derived for the WISSH QSOs with both $\mathrm{H} \beta$ and X-ray measurements, as detailed in Sect. 5.2.

plane $R E W_{\mathrm{CIV}}-v_{\mathrm{CIV}}^{\mathrm{peak}}$ as shown in Fig. 6 and $R E W_{[\mathrm{OIII}]}-v_{\mathrm{CIV}}^{\mathrm{peak}}$ in Fig. 7.

This highlights a dichotomy in the detection of NLR and BLR winds in WISSH QSOs, which could be likely due to inclination effects in a polar geometry scenario for the CIV winds, as suggested in Sect. 4.3.

We found that a sizable fraction of WISSH QSOs exhibit BLR winds traced by CIV blueshifted emission with $\dot{M}_{\text {ion }} \approx 10$ $100 M_{\odot} \mathrm{yr}^{-1}$, comparable with the median accretion rate of WISSH QSOs. Twenty percent of the BLR winds detected in WISSH QSOs show kinetic powers $10^{-3}<\dot{E}_{\text {kin }} / L_{\mathrm{Bol}}<10^{-2}$, which is comparable to those measured for NLR winds associated with [OIII] broad or blueshifted emission lines detected in the $[O I I I]$ sample. The remaining $80 \%$ of the BLR winds show kinetic powers comparable to those traced by [OIII] in less luminous AGNs. This suggests that the BLR winds should be taken into account to obtain a complete census of strong AGNdriven outflows and comprehensively evaluate their effects of depositing energy and momentum into the ISM.

The BLR winds traced by the CIV emission line exhibit a

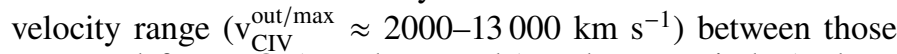
measured for UFO (at sub-pc scale) and NLR winds (at kpcscale), interpreting the outflow velocity distribution as a proxy of the distribution in radial distance for different classes of outflows (see Fig. 11). Moreover, the BLR winds exhibit a $\dot{P}_{\text {out }} / \dot{P}_{\text {AGN }} \lesssim 0.1$, which is lower than that found for UFOs (i.e., $\left.\dot{P}_{\text {out }} / \dot{P}_{\mathrm{AGN}} \sim 1\right)$. In this case the momentum rate of the outflow is comparable to that of the AGN, suggesting a covering factor for the UFOs of the order of unity. Since the covering factor for the BLR is expected to be $\sim 0.1$, we can thus speculate that the momentum load of the nuclear winds may reflect the covering factor of the outflowing gas.

We investigated the dependence of $v_{\mathrm{CIV}}^{\text {peak }}$ on fundamental AGN physical parameters such as $L_{\mathrm{Bol}}, \lambda_{\mathrm{Edd}}$, and $\alpha_{\mathrm{OX}}$. We found a stronger correlation with $L_{\mathrm{Bol}}$ than $\lambda_{\text {Edd }}$ (see Fig. 12), with a dependence consistent with a radiatively-driven winds scenario $\left(v_{\mathrm{CIV}}^{\text {peak }} \propto L_{\mathrm{Bol}}^{0.28 \pm 0.04}\right)$. Moreover, we further studied the dependence of $v_{\mathrm{CIV}}^{\mathrm{peak}}$ on $\alpha_{\mathrm{OX}}$, finding a stronger correlation with $\alpha_{\mathrm{OX}}$ than $L_{\mathrm{Bol}}$. This relation is expected due to the dependence of $\alpha_{\mathrm{OX}}$ on $L_{\mathrm{Bol}}$ (Vignali et al. 2003), that is, when $\alpha_{\mathrm{OX}}$ is steeper, $L_{\mathrm{Bol}}$ is larger. This indicates that the shape of the ionizing continuum could be considered as the primary driver of the blueshifts of the CIV emission line.

Acknowledgements. We thank the anonymous referee for helpful comments that improved the paper. We acknowledge very useful discussions with P. Marziani, B. Husemann and D. Kakkad. We thank M. Vestergaard for kindly providing the UV FeII templates. The scientific results reported in this article are based on observations made by the LBT, the European Southern Observatory (ESO program 093.A-0175(A. B)), and the SDSS. The LBT is an international collaboration among institutions in the United States, Italy, and Germany. LBT Corporation partners are: The University of Arizona on behalf of the Arizona Board of Regents; Istituto Nazionale di Astrofisica, Italy; LBT Beteiligungsgesellschaft, Germany, representing the Max-Planck Society, the Astrophysical Institute Potsdam, and Heidelberg University; The Ohio State University, and The Research Corporation, on behalf of The University of Notre Dame, University of Minnesota and University of Virginia. Funding for the Sloan Digital Sky Survey IV has been provided by the Alfred P. Sloan Foundation, the U.S. Department of Energy Office of Science, and the Participating Institutions. SDSS acknowledges support and resources from the Center for High-Performance Computing at the University of Utah. The SDSS web site is https://www.sdss.org/. The SDSS is managed by the Astrophysical Research Consortium for the Participating Institutions. The Participating Institutions are the American Museum of Natural History, Astrophysical Institute Potsdam, University of Basel, University of Cambridge, Case Western Reserve University, University of Chicago, Drexel University, Fermilab, the Institute for Advanced Study, the Japan Participation Group, Johns Hopkins University, the Joint Institute for Nuclear Astrophysics, the Kavli Institute for Particle Astrophysics and Cosmology, the Korean Scientist Group, the Chinese Academy of Sciences (LAMOST), Los Alamos National Laboratory, the Max-Planck-Institute for Astronomy (MPIA), the Max-Planck-Institute for Astrophysics (MPA), New Mexico State University, Ohio State University, University of Pittsburgh, University of Portsmouth, Princeton University, the United States Naval Observatory, and the University of Washington. This research has made use of the NASA/IPAC Extragalactic Database (NED), which is operated by the Jet Propulsion Laboratory, California Institute of Technology, under contract with the National Aeronautics and Space Administration. This research project was supported by the DFG Cluster of Excellence "Origin and Structure of the Universe" (www.universe-cluster.de). We acknowledge financial support from PRIN-INAF 2014 (Windy Black Holes combing galaxy evolution). A. Bongiorno and E. Piconcelli acknowledge financial support from INAF under the contract PRIN-INAF-2012. L. Zappacosta acknowledges financial support under ASI/INAF contract I/037/12/0. G. Bruni acknowledges financial support under the INTEGRAL ASI-INAF agreement 2013-025.R01. F. Tombesi acknowledges support from the 2014 MIUR grant "Rita Levi Montalcini". S. Bisogni is supported by the National Aeronautics and Space Administration through Chandra Award Number AR7-18013 X issued by the Chandra X-ray Observatory Center, which is operated by the Smithsonian Astrophysical Observatory for and on behalf of the National Aeronautics Space Administration under contract NAS8-03060. We are grateful to M. Fumana for his assistance in LBT data reduction.

\section{References}

Ahn, C. P., Alexandroff, R., Allende Prieto, C., et al. 2014, ApJS, 211, 17 Baskin, A., \& Laor, A. 2005, MNRAS, 356, 1029

Bentz, M. C., Peterson, B. M., Netzer, H., Pogge, R. W., \& Vestergaard, M. 2009, ApJ, 697, 160

Bernardi, M., Sheth, R. K., Nichol, R. C., Schneider, D. P., \& Brinkmann, J. 2005, AJ, 129, 61

Bischetti, M., Piconcelli, E., Vietri, G., et al. 2017, A\&A, 598, A122

Bisogni, S., Marconi, A., \& Risaliti, G. 2017a, MNRAS, 464, 385

Bisogni, S., Marconi, A., Risaliti, G., \& Lusso, E. 2017b, Fr. Astron. Space Sci. 4, 48

Bongiorno, A., Maiolino, R., Brusa, M., et al. 2014, MNRAS, 443, 2077

Boroson, T. A. 2002, ApJ, 565, 78

Boroson, T. A., \& Green, R. F. 1992, ApJS, 80, 109

Bruni, G., Mack, K.-H., Salerno, E., et al. 2012, A\&A, 542, A13

Brusa, M., Bongiorno, A., Cresci, G., et al. 2015, MNRAS, 446, 2394

Cano-Díaz, M., Maiolino, R., Marconi, A., et al. 2012, A\&A, 537, L8

Carniani, S., Marconi, A., Maiolino, R., et al. 2015, A\&A, 580, A102

Cicone, C., Maiolino, R., Sturm, E., et al. 2014, A\&A, 562, A21

Coatman, L., Hewett, P. C., Banerji, M., \& Richards, G. T. 2016, MNRAS, 461, 647 
Coatman, L., Hewett, P. C., Banerji, M., et al. 2017, MNRAS, 465, 2120

Corbin, M. R., \& Boroson, T. A. 1996, ApJS, 107, 69

Cresci, G., Mainieri, V., Brusa, M., et al. 2015, ApJ, 799, 82

Davies, R. I. 2007, MNRAS, 375, 1099

Denney, K. D. 2012, ApJ, 759, 44

Di Matteo, T., Springel, V., \& Hernquist, L. 2005, Nature, 433, 604

Diamond-Stanic, A. M., Fan, X., Brandt, W. N., et al. 2009, ApJ, 699, 782

Duras, F., Bongiorno, A., Piconcelli, E., et al. 2017, A\&A, 604, A67

Fabian, A. C. 2012, ARA\&A, 50, 455

Fan, X., Strauss, M. A., Gunn, J. E., et al. 1999, ApJ, 526, L57

Faucher-Giguère, C.-A., \& Quataert, E. 2012, MNRAS, 425, 605

Ferland, G. J., \& Elitzur, M. 1984, ApJ, 285, L11

Ferland, G. J., Porter, R. L., van Hoof, P. A. M., et al. 2013, Rev. Mexicana Astron. Astrofis., 49, 137

Feruglio, C., Maiolino, R., Piconcelli, E., et al. 2010, A\&A, 518, L155

Feruglio, C., Fiore, F., Carniani, S., et al. 2015, A\&A, 583, A99

Fiore, F., Feruglio, C., Shankar, F., et al. 2017, A\&A, 601, A143

Freudling, W., Romaniello, M., Bramich, D. M., et al. 2013, A\&A, 559, A96

Gaskell, C. M. 1982, ApJ, 263, 79

Genzel, R., Förster Schreiber, N. M., Rosario, D., et al. 2014, ApJ, 796, 7

Gofford, J., Reeves, J. N., Tombesi, F., et al. 2013, MNRAS, 430, 60

Gofford, J., Reeves, J. N., McLaughlin, D. E., et al. 2015, MNRAS, 451, 4169

Granato, G. L., De Zotti, G., Silva, L., Bressan, A., \& Danese, L. 2004, ApJ, 600, 580

Gunn, J. E., Siegmund, W. A., Mannery, E. J., et al. 2006, AJ, 131, 2332

Hall, P. B., Anderson, S. F., Strauss, M. A., et al. 2002, ApJS, 141, 267

Hamann, F., Zakamska, N. L., Ross, N., et al. 2017, MNRAS, 464, 3431

Harrison, C. M., Alexander, D. M., Swinbank, A. M., et al. 2012, MNRAS, 426, 1073

Harrison, C. M., Alexander, D. M., Mullaney, J. R., \& Swinbank, A. M. 2014, MNRAS, 441, 3306

Hewett, P. C., \& Wild, V. 2010, MNRAS, 405, 2302

Just, D. W., Brandt, W. N., Shemmer, O., et al. 2007, ApJ, 665, 1004

Kashi, A., Proga, D., Nagamine, K., Greene, J., \& Barth, A. J. 2013, ApJ, 778, 50

Kaspi, S., Smith, P. S., Netzer, H., et al. 2000, ApJ, 533, 631

King, A., \& Pounds, K. 2015, ARA\&A, 53, 115

King, A. R. 2010, MNRAS, 402, 1516

Kormendy, J., \& Richstone, D. 1995, ARA\&A, 33, 581

Laor, A., \& Brandt, W. N. 2002, ApJ, 569, 641

Leighly, K. M. 2004, ApJ, 611, 125

Luo, B., Brandt, W. N., Hall, P. B., et al. 2015, ApJ, 805, 122

Lusso, E., Comastri, A., Vignali, C., et al. 2010, A\&A, 512, A34

Markwardt, C. B. 2009, in Astronomical Data Analysis Software and Systems XVIII, eds. D. A. Bohlender, D. Durand, \& P. Dowler, ASP Conf. Ser., 411, 251

Martocchia, S., Piconcelli, E., Zappacosta, L., et al. 2017, A\&A, 608, A51

Marziani, P., Sulentic, J. W., Zwitter, T., Dultzin-Hacyan, D., \& Calvani, M. 2001, ApJ, 558, 553

Marziani, P., Sulentic, J. W., Stirpe, G. M., Zamfir, S., \& Calvani, M. 2009, A\&A, 495, 83

Marziani, P., Sulentic, J. W., Negrete, C. A., et al. 2010, MNRAS, 409, 1033

Marziani, P., Martínez Carballo, M. A., Sulentic, J. W., et al. 2016, Ap\&SS, 361, 29

Matsuoka, K., Silverman, J. D., Schramm, M., et al. 2013, ApJ, 771, 64
McGill, K. L., Woo, J.-H., Treu, T., \& Malkan, M. A. 2008, ApJ, 673, 703 Menci, N., Fiore, F., Puccetti, S., \& Cavaliere, A. 2008, ApJ, 686, 219

Nagao, T., Marconi, A., \& Maiolino, R. 2006, A\&A, 447, 157

Nardini, E., Reeves, J. N., Gofford, J., et al. 2015, Science, 347, 860

Netzer, H. 1990, in Active Galactic Nuclei, eds. R. D. Blandford, H. Netzer, L. Woltjer, T. J.-L. Courvoisier, \& M. Mayor, (Berlin: Springer-Verlag), 57 Netzer, H., \& Laor, A. 1993, ApJ, 404, L51

Peterson, B. M. 1997, An Introduction to Active Galactic Nuclei (Cambridge, New York: Cambridge University Press)

Plotkin, R. M., Anderson, S. F., Brandt, W. N., et al. 2010, ApJ, 721, 562

Plotkin, R. M., Shemmer, O., Trakhtenbrot, B., et al. 2015, ApJ, 805, 123

Prevot, M. L., Lequeux, J., Prevot, L., Maurice, E., \& Rocca-Volmerange, B. 1984, A\&A, 132, 389

Ricci, F., La Franca, F., Onori, F., \& Bianchi, S. 2017, A\&A, 598, A51

Richards, G. T., Vanden Berk, D. E., Reichard, T. A., et al. 2002, AJ, 124, 1

Richards, G. T., Lacy, M., Storrie-Lombardi, L. J., et al. 2006, ApJS, 166, 470

Richards, G. T., Kruczek, N. E., Gallagher, S. C., et al. 2011, AJ, 141, 167

Risaliti, G., Salvati, M., \& Marconi, A. 2011, MNRAS, 411, 2223

Runnoe, J. C., Brotherton, M. S., \& Shang, Z. 2012, MNRAS, 427, 1800

Rupke, D. S. N., \& Veilleux, S. 2015, ApJ, 801, 126

Shen, Y. 2016, ApJ, 817, 55

Shen, Y., \& Ho, L. C. 2014, Nature, 513, 210

Shen, Y., \& Liu, X. 2012, ApJ, 753, 125

Shen, Y., Richards, G. T., Strauss, M. A., et al. 2011, ApJS, 194, 45

Silk, J., \& Rees, M. J. 1998, A\&A, 331, L1

Skrutskie, M. F., Cutri, R. M., Stiening, R., et al. 2006, AJ, 131, 1163

Spoon, H. W. W., Farrah, D., Lebouteiller, V., et al. 2013, ApJ, 775, 127

Steffen, A. T., Strateva, I., Brandt, W. N., et al. 2006, AJ, 131, 2826

Sulentic, J. W., Marziani, P., \& Dultzin-Hacyan, D. 2000, ARA\&A, 38, 521

Sulentic, J. W., Stirpe, G. M., Marziani, P., et al. 2004, A\&A, 423, 121

Sulentic, J. W., Repetto, P., Stirpe, G. M., et al. 2006, A\&A, 456, 929

Sulentic, J. W., Bachev, R., Marziani, P., Negrete, C. A., \& Dultzin, D. 2007, ApJ, 666, 757

Sulentic, J. W., del Olmo, A., Marziani, P., et al. 2017, A\&A, 608, A122

Tang, B., Shang, Z., Gu, Q., Brotherton, M. S., \& Runnoe, J. C. 2012, ApJS, 201, 38

Tombesi, F., Cappi, M., Reeves, J. N., et al. 2010, A\&A, 521, A57

Tombesi, F., Cappi, M., Reeves, J. N., \& Braito, V. 2012, MNRAS, 422, L1

Tombesi, F., Cappi, M., Reeves, J. N., et al. 2013, MNRAS, 430, 1102

Tombesi, F., Tazaki, F., Mushotzky, R. F., et al. 2014, MNRAS, 443, 2154

Tombesi, F., Meléndez, M., Veilleux, S., et al. 2015, Nature, 519, 436

Trakhtenbrot, B., \& Netzer, H. 2012, MNRAS, 427, 3081

Tsuzuki, Y., Kawara, K., Yoshii, Y., et al. 2006, ApJ, 650, 57

Vacca, W. D., Cushing, M. C., \& Rayner, J. T. 2003, PASP, 115, 389

Veilleux, S., Bolatto, A., Tombesi, F., et al. 2017, ApJ, 843, 18

Véron-Cetty, M.-P., Joly, M., \& Véron, P. 2004, A\&A, 417, 515

Vestergaard, M., \& Wilkes, B. J. 2001, ApJS, 134, 1

Vignali, C., Brandt, W. N., \& Schneider, D. P. 2003, AJ, 125, 433

Weymann, R. J., Morris, S. L., Foltz, C. B., \& Hewett, P. C. 1991, ApJ, 373, 23

Wu, J., Vanden Berk, D. E., Brandt, W. N., et al. 2009, ApJ, 702, 767

Wu, J., Brandt, W. N., Hall, P. B., et al. 2011, ApJ, 736, 28

Young, S., Axon, D. J., Robinson, A., Hough, J. H., \& Smith, J. E. 2007, Nature, 450,74

Zubovas, K., \& King, A. 2012, ApJ, 745, L34 


\section{Appendix A: SDSS J1538+0855}

We present here a detailed analysis of the source J1538+0855 since it is the only object exhibiting both NLR and BLR winds. As reported in Sect. 4.3.2, we measured the CIV outflow properties based on the so-called outflow component. We estimated the gas mass of the outflow as $M_{\text {ion }}=590 M_{\odot}$, the maximum velocity of the outflow as representative of the bulk velocity of the outflow, $v_{\mathrm{CIV}}^{\max }=13400 \mathrm{~km} \mathrm{~s}^{-1}$, the ionized gas mass rate as $\dot{M}_{\text {ion }}=$ $24 M_{\odot} \mathrm{yr}^{-1}$, and the kinetic power as $\dot{E}_{\text {kin }}=1.4 \times 10^{45} \mathrm{erg} \mathrm{s}^{-1}$. We also measured the properties of the CIV outflow considering the entire CIV profile, with the $v_{\mathrm{CIV}}^{\text {peak }}$ as representative of the outflow velocity. We estimated $M_{\text {ion }}=1210 M_{\odot}$, a ionized gas mass rate $\dot{M}_{\text {ion }}=8 M_{\odot} \mathrm{yr}^{-1}$, and a kinetic power $\dot{E}_{\text {kin }}=12 \times 10^{42} \mathrm{erg} \mathrm{s}^{-1}$.

As mentioned in Sect. 3, we found a broad $\left(\sim 2000 \mathrm{~km} \mathrm{~s}^{-1}\right)$, blueshifted (4987 ̊) [OIII] component, indicative of outflow. In order to characterize the ionized outflowing gas, we calculated the mass rate $\dot{M}$ and the kinetic power $\dot{E}_{\text {kin }}$, assuming the outflow model discussed in Paper I and using the [OIII] emission line. According to Equations (2), (5), and (6) in Paper I, we derived $M_{\text {ion }}^{[\mathrm{OIII}]}=4.2 \times 10^{8} M_{\odot}, \dot{M}_{\text {ion }}^{[\mathrm{OIII}]}=530 M_{\odot} \mathrm{yr}^{-1}$, and $\dot{E}_{\text {kin }}^{[\mathrm{OIII}]}=1.4 \times 10^{45} \mathrm{erg} \mathrm{s}^{-1}$ of the ionized outflow, assuming an electron density of $n_{\mathrm{e}}=200 \mathrm{~cm}^{-3}$, a solar metallicity, and an outflow velocity defined as $v_{\max }=|\Delta v|+2 \sigma_{\text {[OIII] }}^{\text {broad }}$, where $\Delta v$ is the velocity shift between the broad [OIII] component and the systemic [OIII] assumed at $5007 \AA$, and $\sigma_{\text {[OIII] }}^{\text {broad }}$ is the velocity dispersion of the broad [OIII] component $\left(\mathrm{v}_{\max }=2900 \mathrm{~km} \mathrm{~s}^{-1}\right)$. We refer to Paper I for more details about the assumptions used in the calculation of the outflow parameters.

We investigated how the values of $\dot{M}_{\text {ion }}^{[\mathrm{OIII}]}$ and $\dot{E}_{\mathrm{kin}}^{[\mathrm{OIII}]}$ are sensitive to different assumed parameters. As in paper I, the upper bound corresponds to the assumption of $\mathrm{n}_{e}=$ $80 \mathrm{~cm}^{-3}$ (as in Genzel et al. 2014) and we determined that $\dot{M}_{\text {ion }}^{[\mathrm{OIII}]}=1330 M_{\odot} \mathrm{yr}^{-1}$ and $\dot{E}_{\mathrm{kin}}^{[\mathrm{OIII}]}=3.5 \times 10^{45} \mathrm{erg} \mathrm{s}^{-1}$, while the lower bound corresponds to $\mathrm{n}_{e}=1000 \mathrm{~cm}^{-3}$ (typical value for the NLR, Peterson 1997) and a velocity of $W_{80} / 1.3$ (as in Harrison et al. 2014), where $W_{80}$ is the velocity width of the line at $80 \%$ of the line flux. We derived a lower bound of $\dot{M}_{\text {ion }}^{[\mathrm{OIII}]}=60 M_{\odot} \mathrm{yr}^{-1}$ and $\dot{E}_{\text {kin }}^{[\mathrm{OIII}]}=$ $5 \times 10^{43} \mathrm{erg} \mathrm{s}^{-1}$.

\section{Appendix B: Optical rest-frame spectra of 13 WISSH QSOs}

We present the LBT/LUCI and SINFONI optical rest-frame spectra in the wavelength range of the $\mathrm{H} \beta$-[OIII] emission lines for the 13 WISSH QSOs analyzed in the present paper (see Table 1).
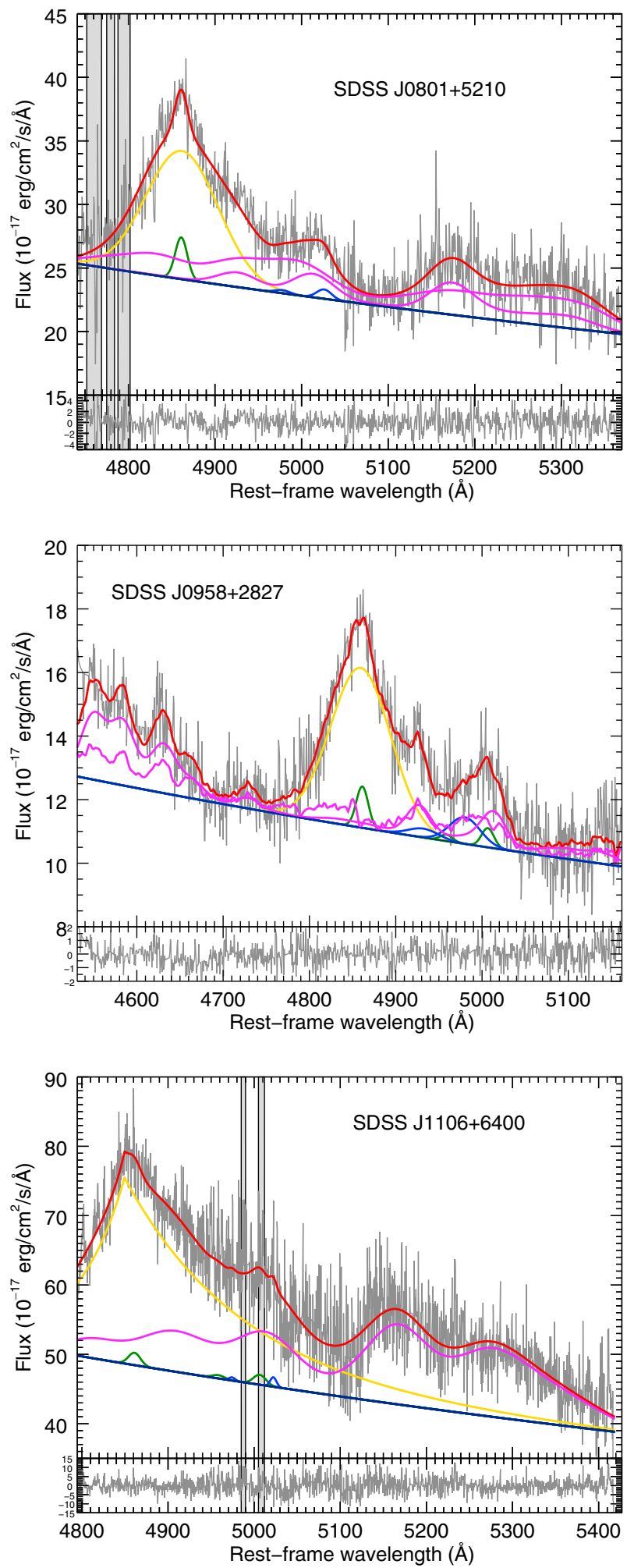

Fig. B.1. The red line shows the best fit of the spectrum. Green curve refers to the core component associated with the NLR emission of $\mathrm{H} \beta$ and [OIII] emission lines. Blue curve refers to the broad blueshifted emission of [OIII] $\lambda \lambda 4959,5007 \AA$, indicative of outflow. Gold curve indicates the broad component of $\mathrm{H} \beta$ associated with BLR emission. FeII emission is indicated in magenta. Grey band indicates the region excluded from the fit because of the presence of sky line residual. Lower panel shows the fit residuals. 

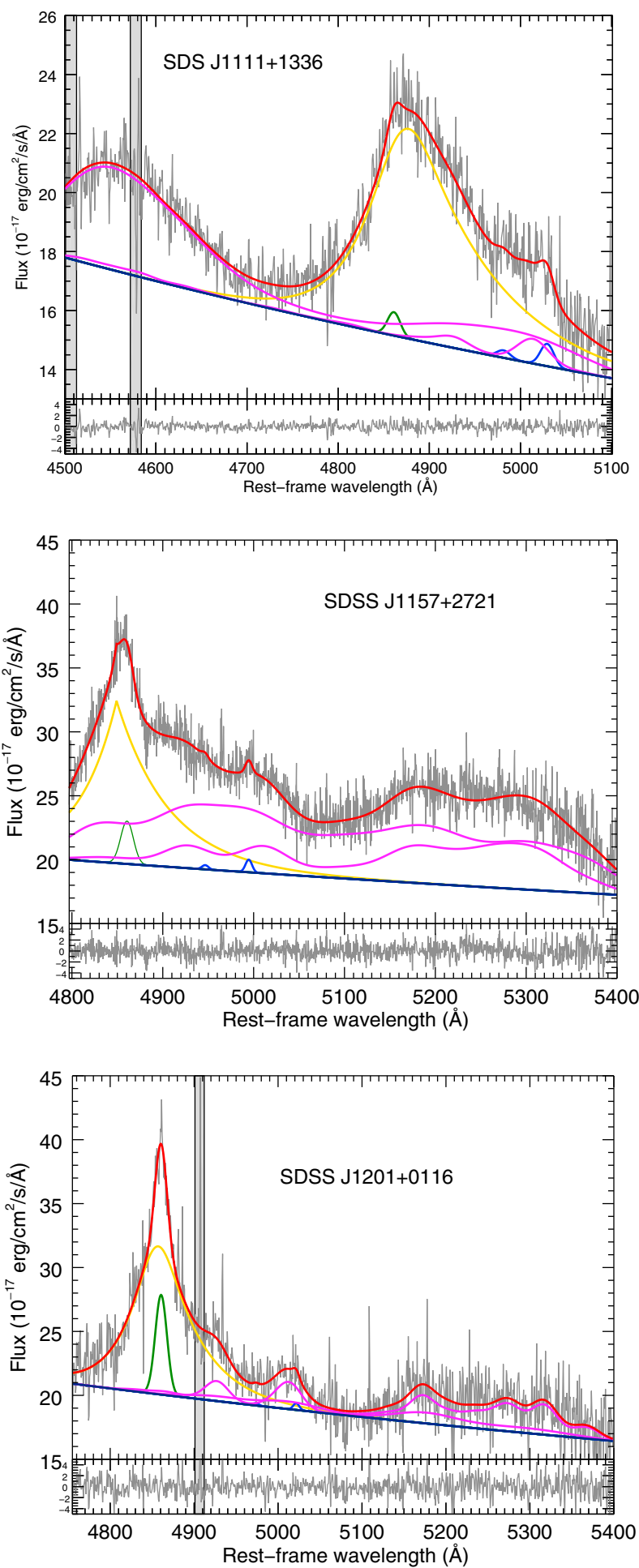

Fig. B.1. continued.
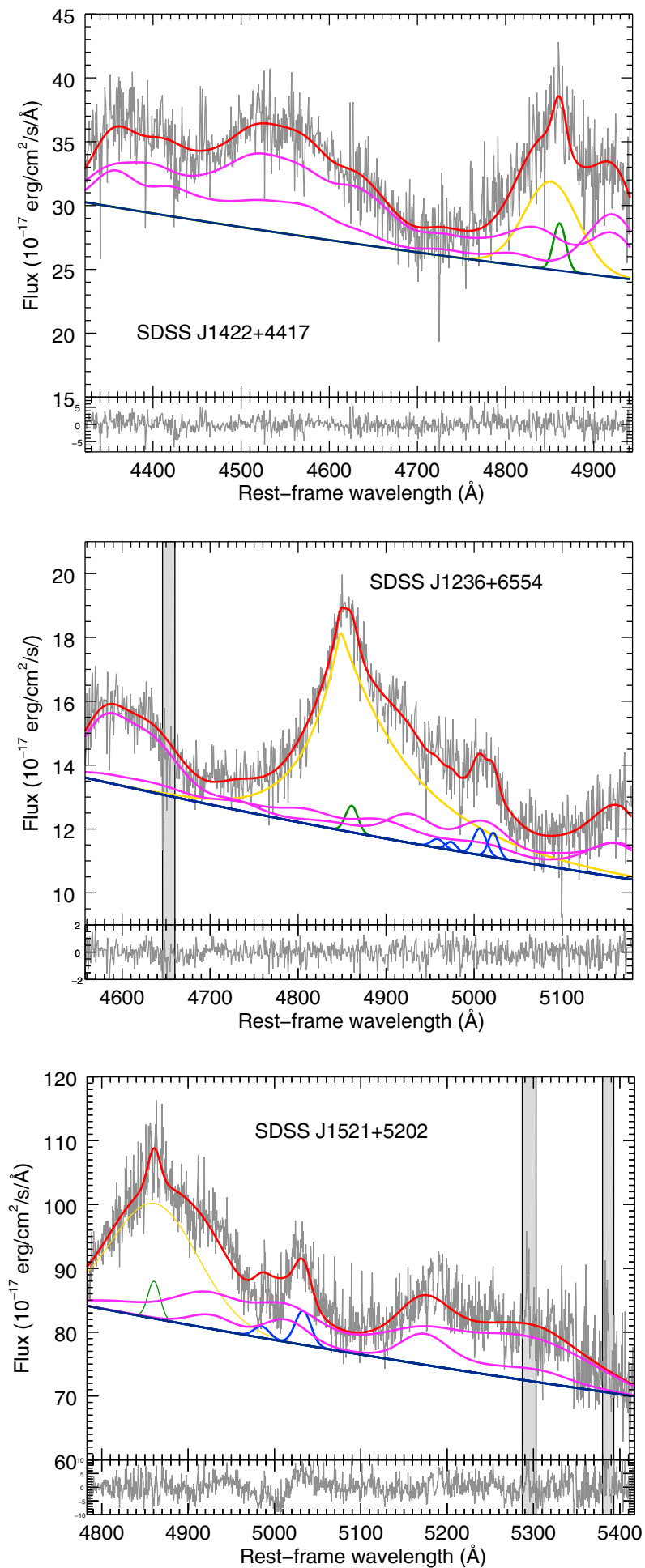

Fig. B.1. continued. 
G. Vietri et al.: The WISSH quasars project
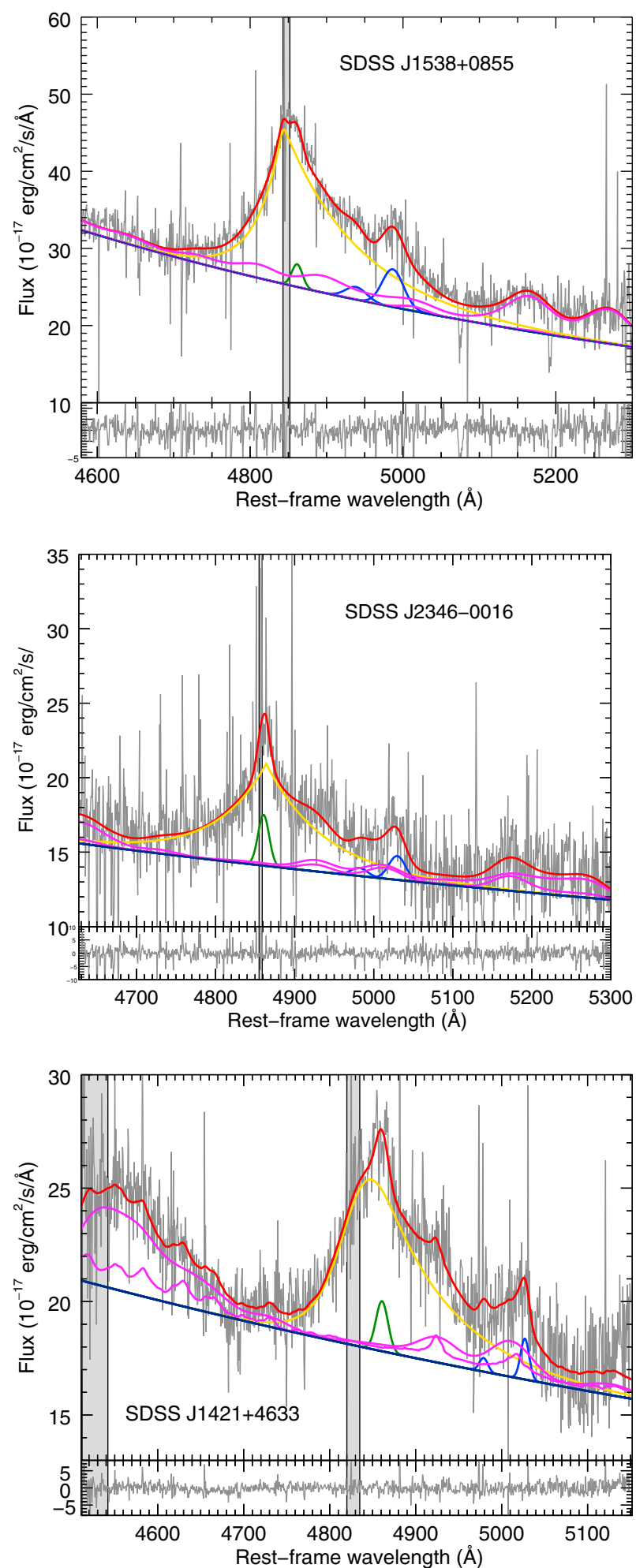

Fig. B.1. continued.

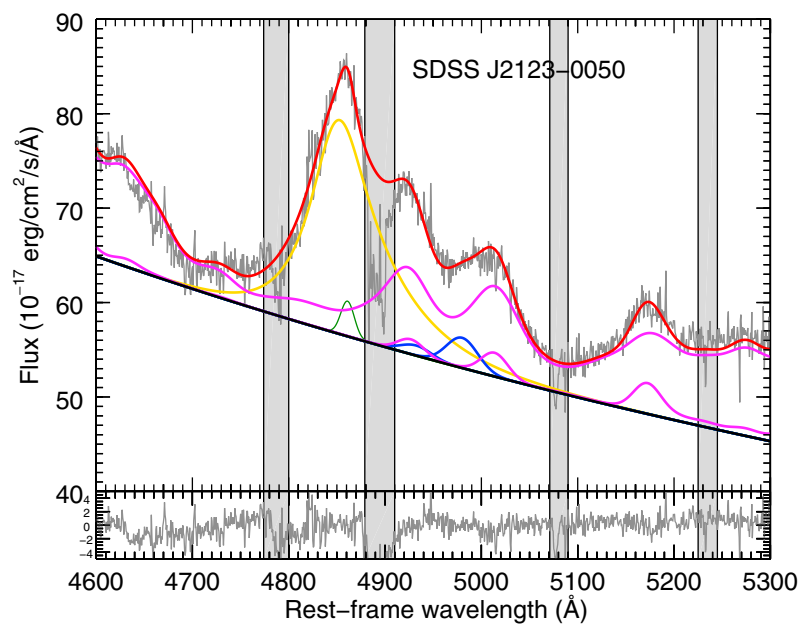

Fig. B.1. continued.

\section{Appendix C: SDSS Optical/UV rest-frame spectra of 18 WISSH QSOs}

We present the SDSS DR10 UV rest-frame spectra of the 18 WISSH QSOs analyzed in the present paper.

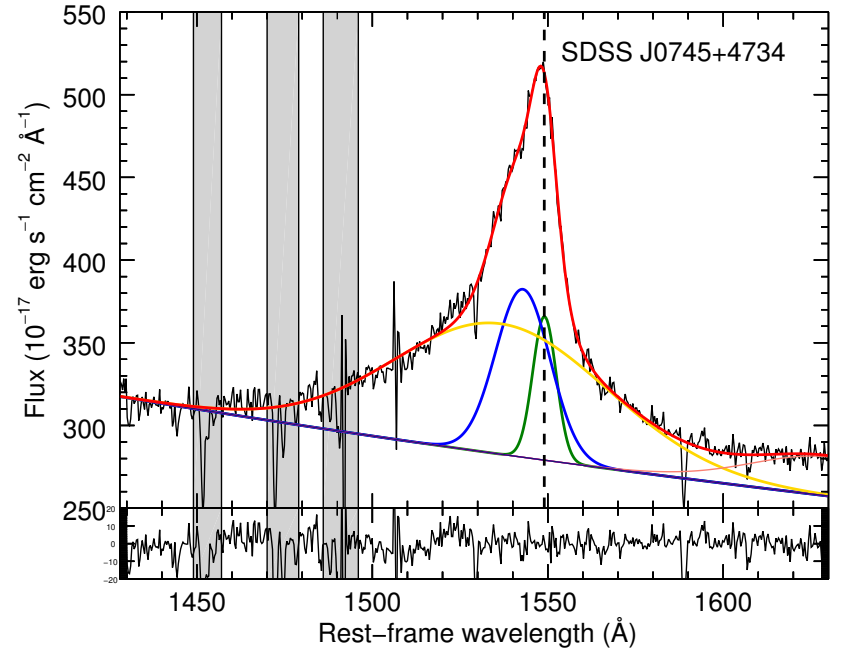

Fig. C.1. SDSS DR10 UV rest-frame spectra. The red line shows the best-fit of the spectrum. Green curve refers to the core component likely associated with the NLR emission of the CIV emission. Blue curve refers to the broad blueshifted emission of CIV emission line, indicative of outflow. Gold curve indicates the broad virialized component associated with the BLR emission. FeII emission is indicated in magenta. Grey bands indicate the region excluded from the fit because of the presence of absorption lines. The dashed line denotes the CIV $\lambda 1549 \AA$ A rest-frame wavelength. Lower panel shows the fit residuals. 


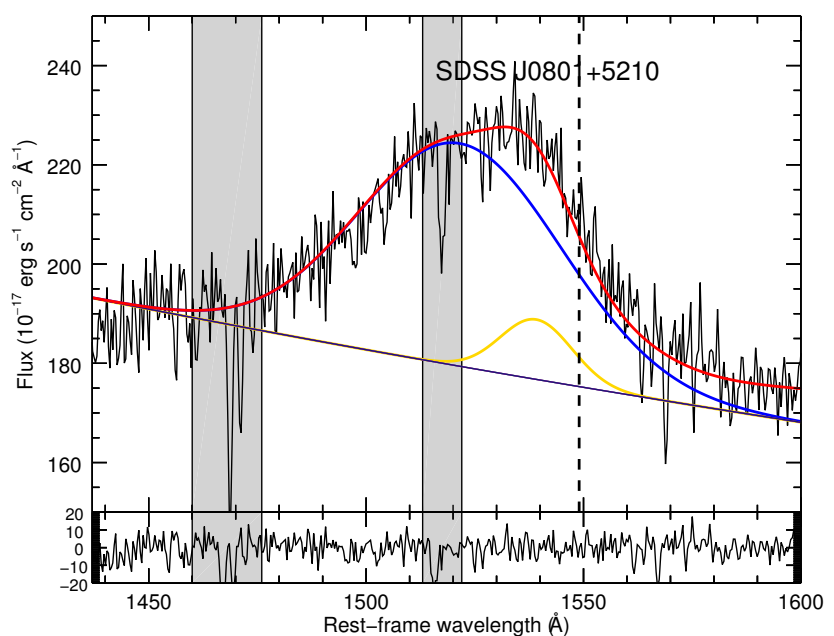

Fig. C.2. Same legend as in Fig. C.1.

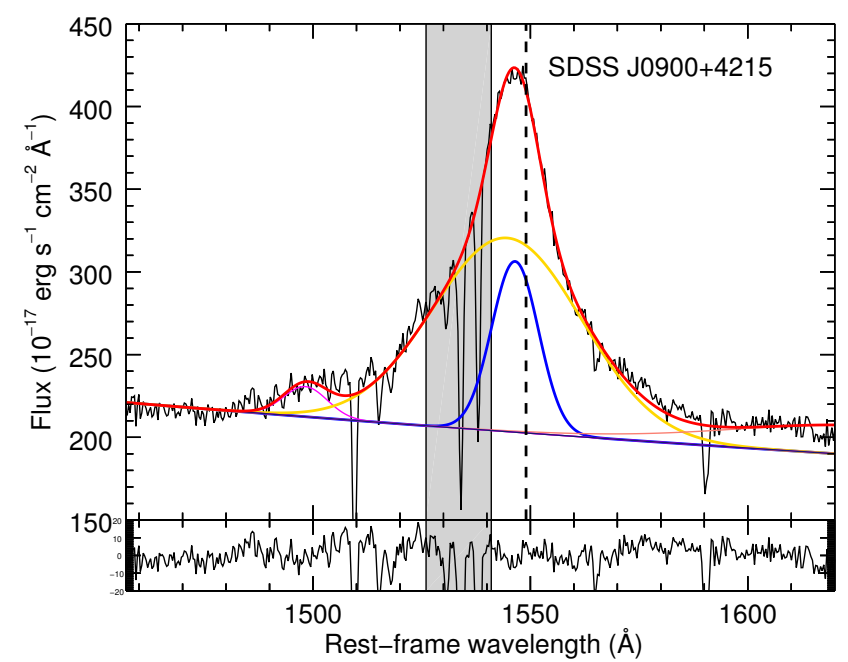

Fig. C.3. Same legend as in Fig. C.1.

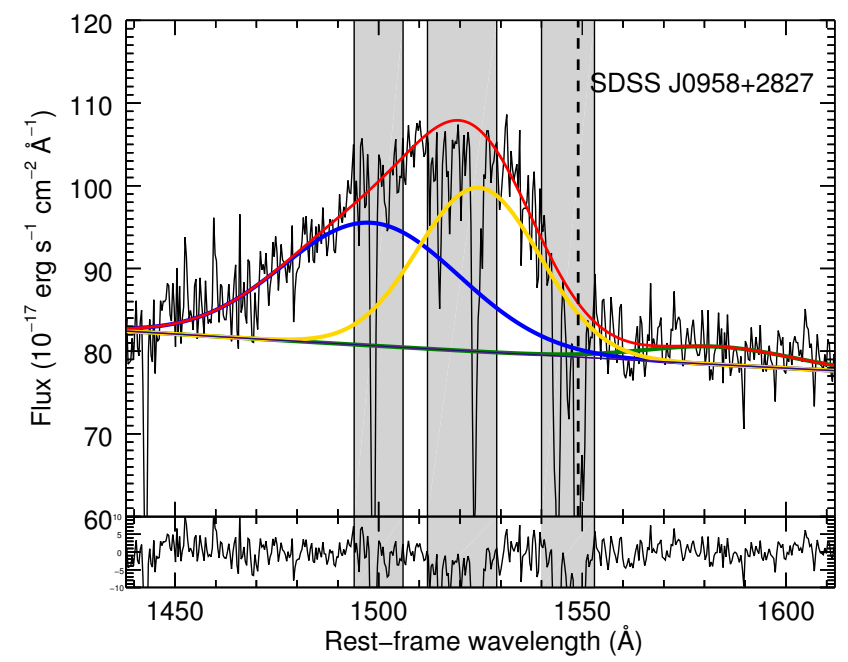

Fig. C.4. Same legend as in Fig. C.1.

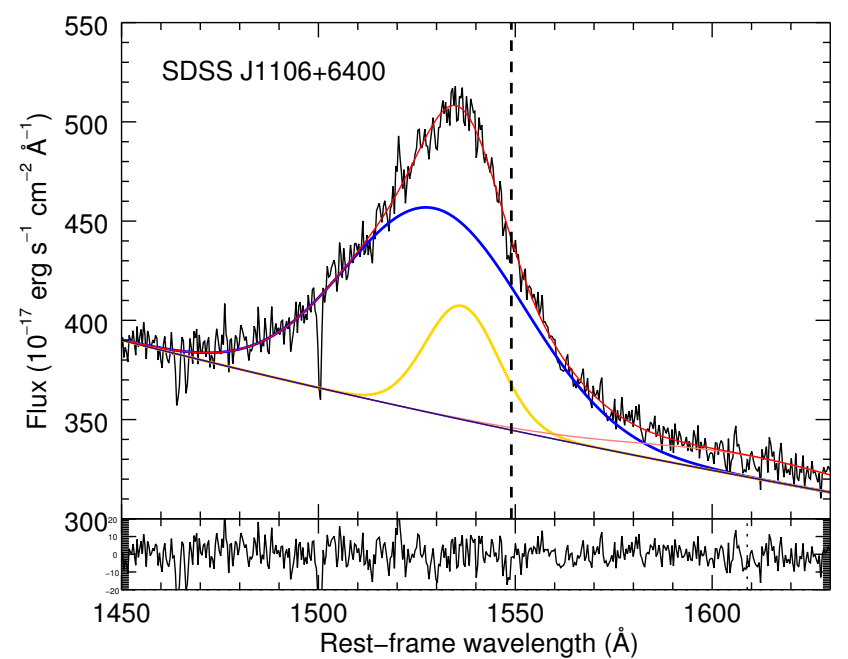

Fig. C.5. Same legend as in Fig. C.1.

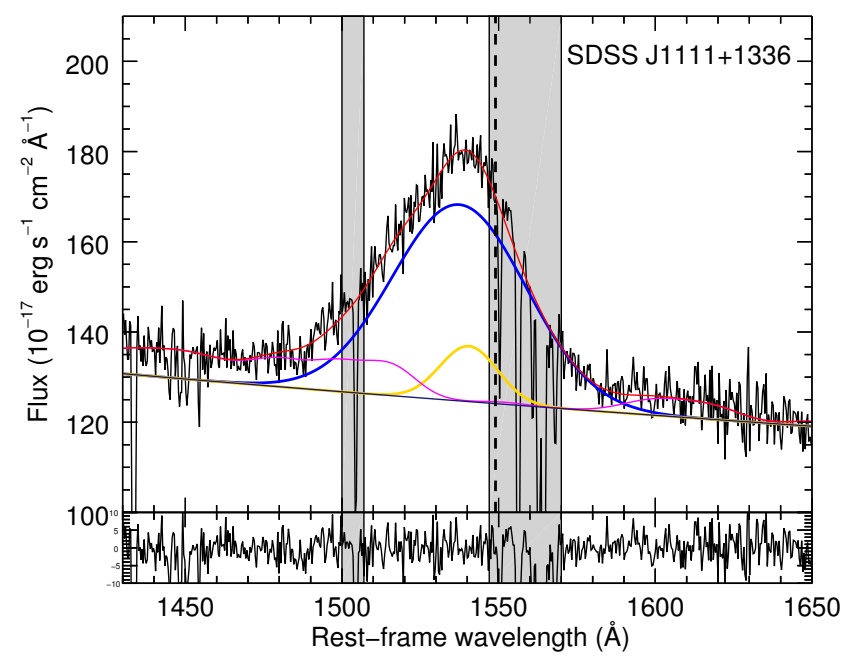

Fig. C.6. Same legend as in Fig. C.1.

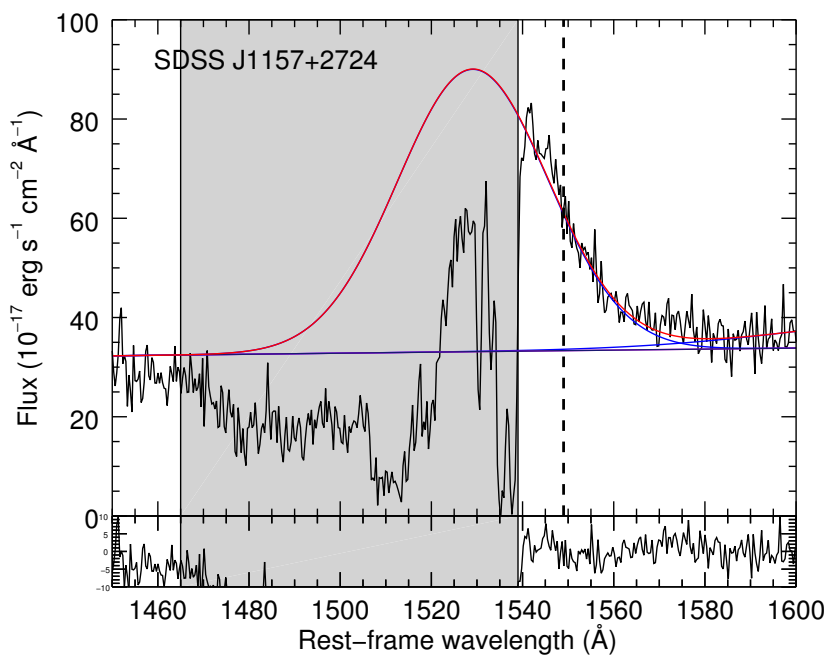

Fig. C.7. Same legend as in Fig. C.1. 
G. Vietri et al.: The WISSH quasars project

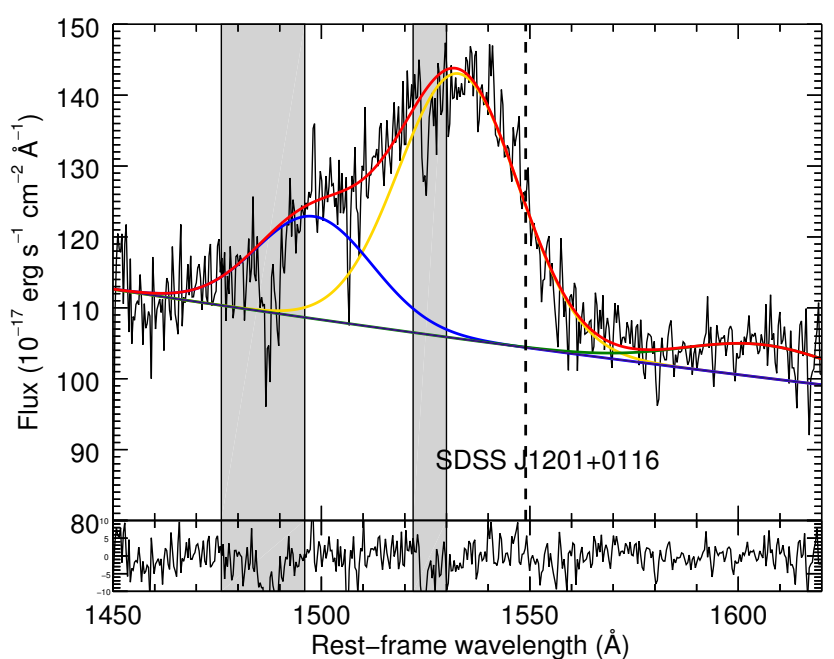

Fig. C.8. Same legend as in Fig. C.1.

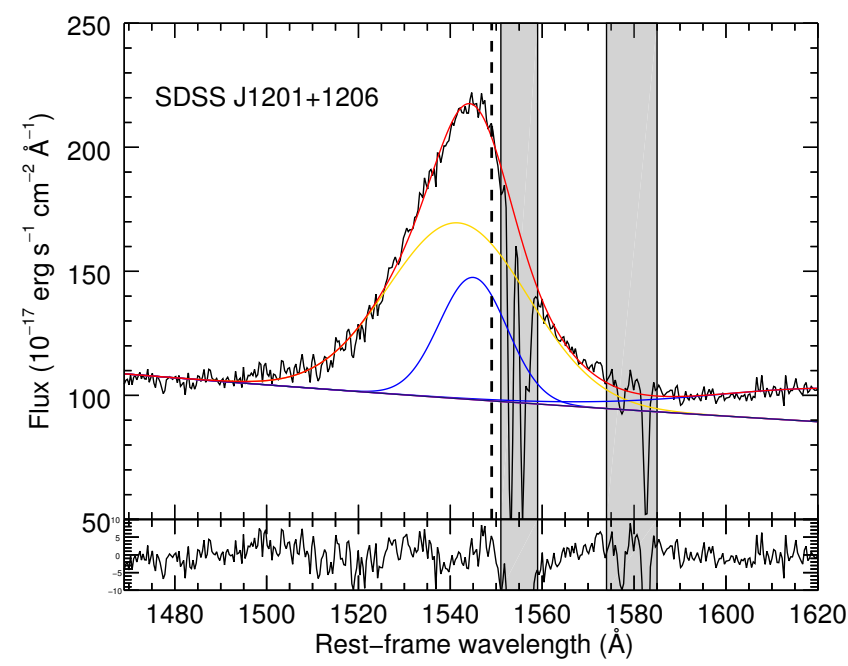

Fig. C.9. Same legend as in Fig. C.1.

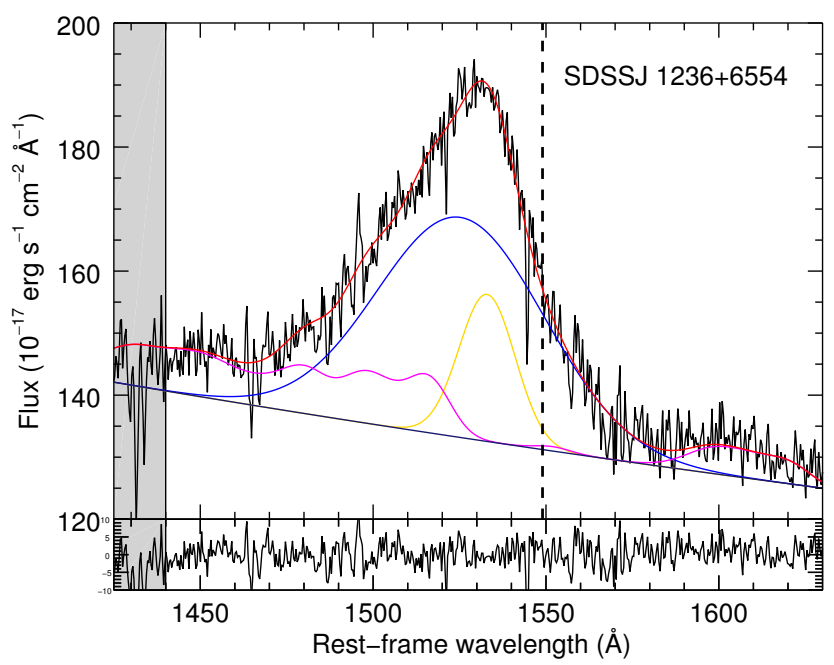

Fig. C.10. Same legend as in Fig. C.1.

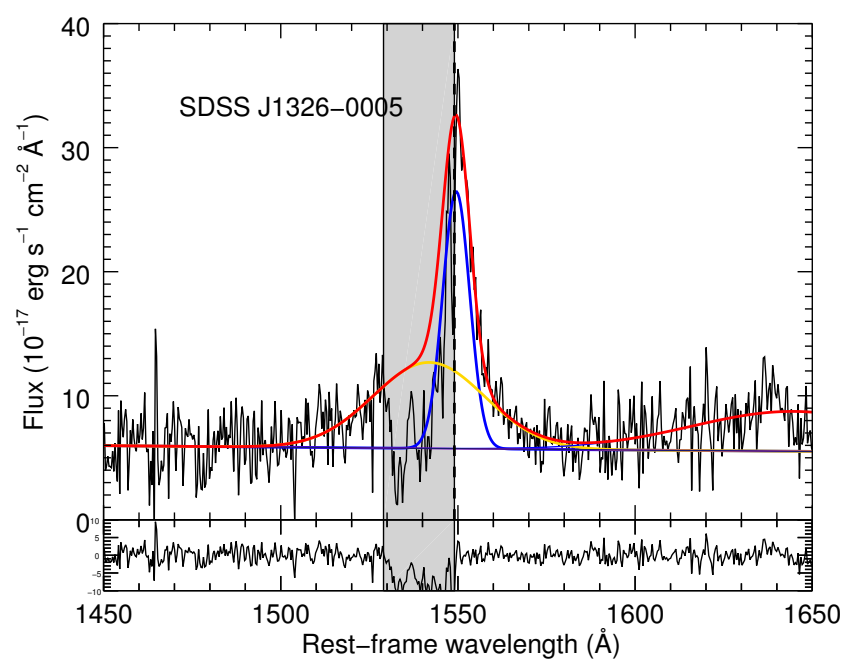

Fig. C.11. Same legend as in Fig. C.1.

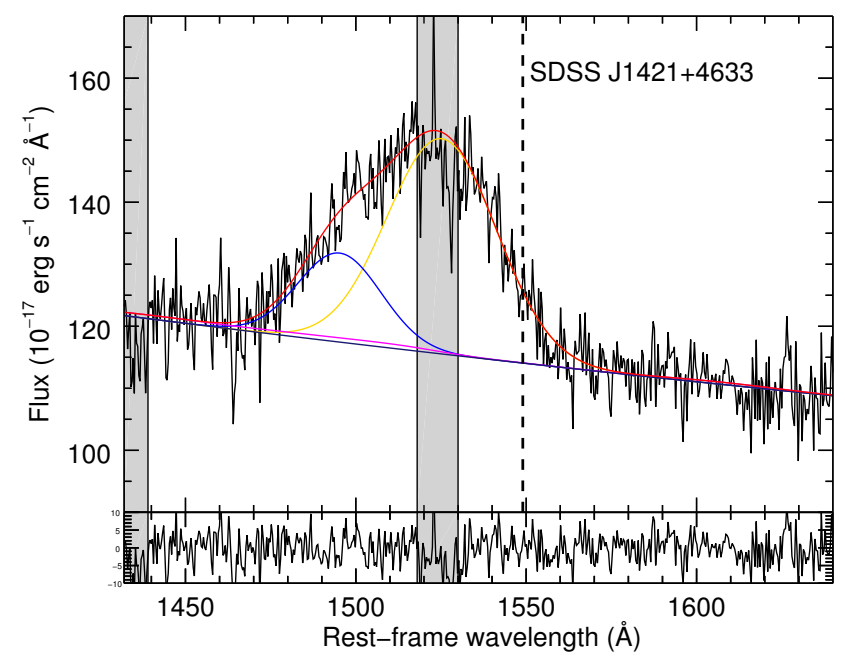

Fig. C.12. Same legend as in Fig. C.1.

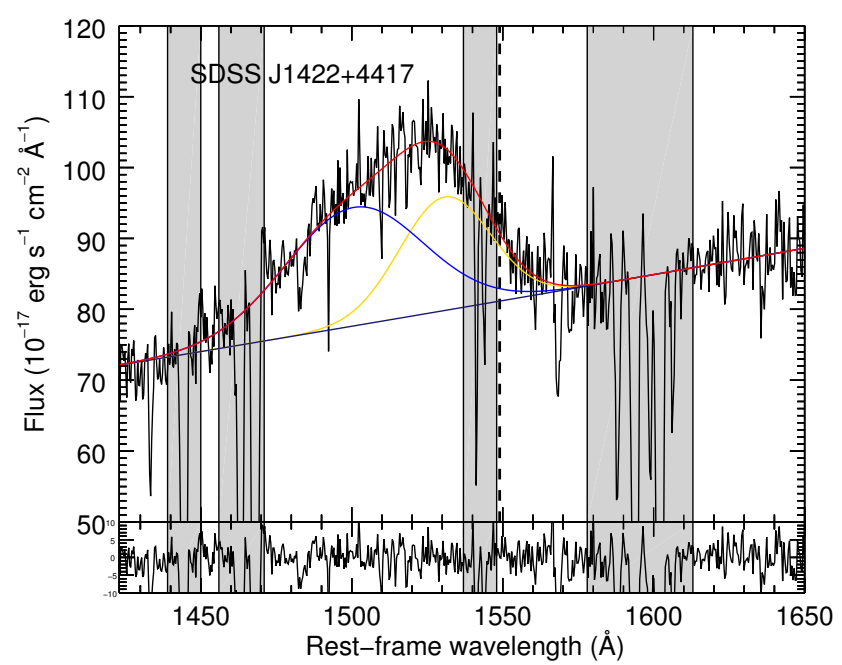

Fig. C.13. Same legend as in Fig. C.1. 


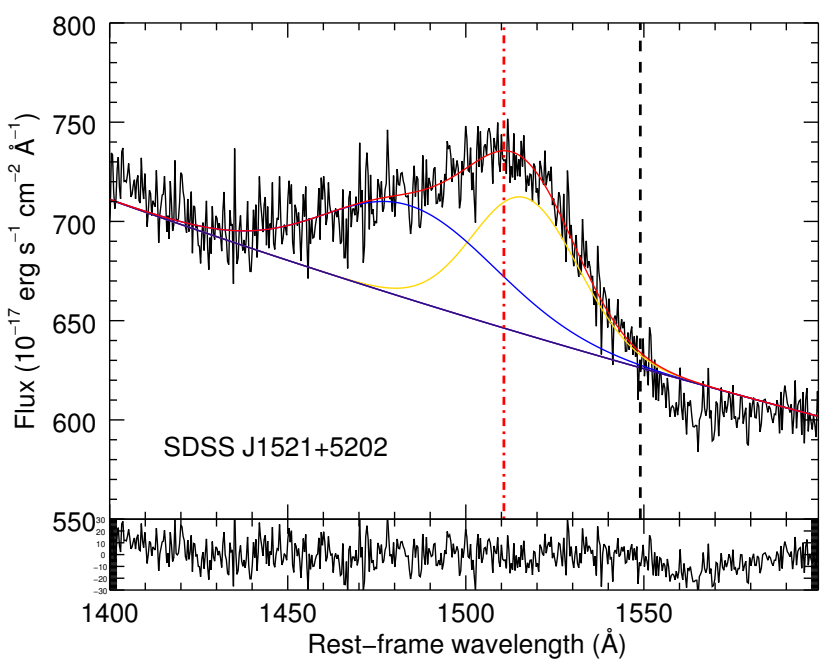

Fig. C.14. Same legend as in Fig. C.1.

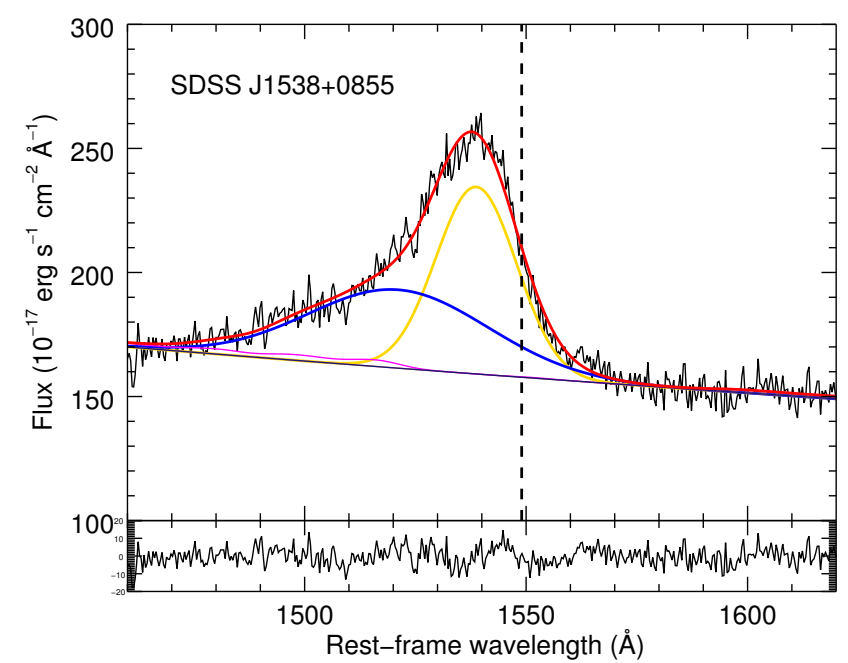

Fig. C.15. Same legend as in Fig. C.1.

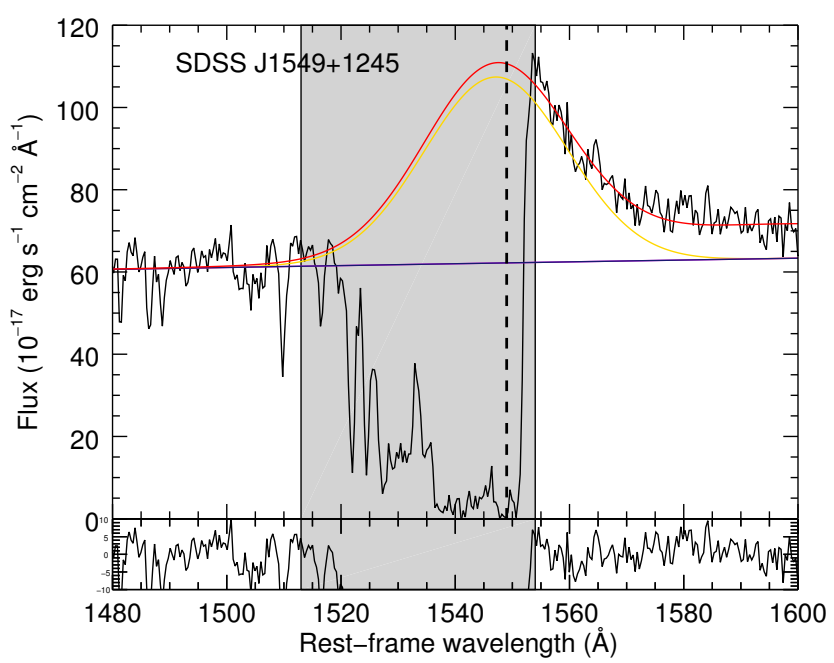

Fig. C.16. Same legend as in Fig. C.1.

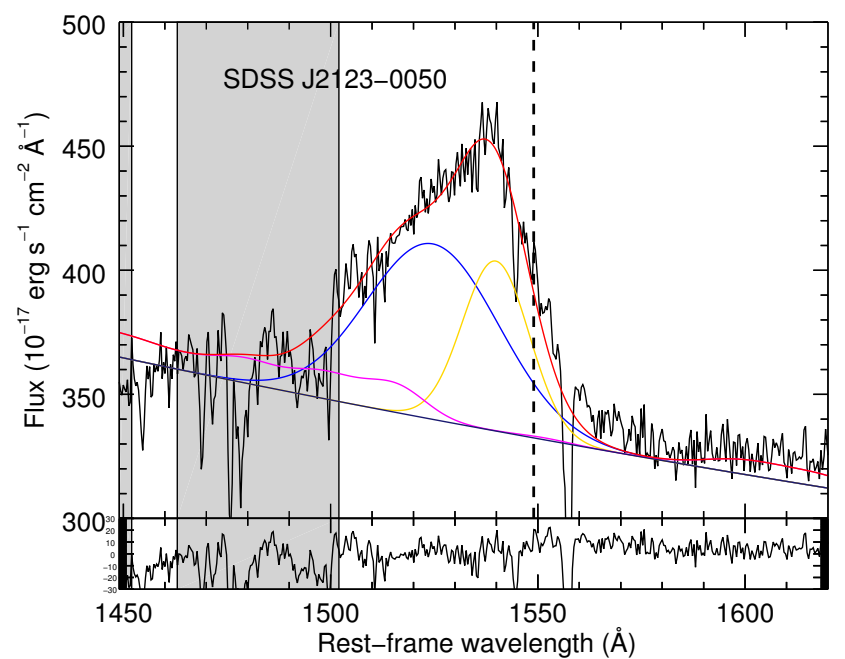

Fig. C.17. Same legend as in Fig. C.1.

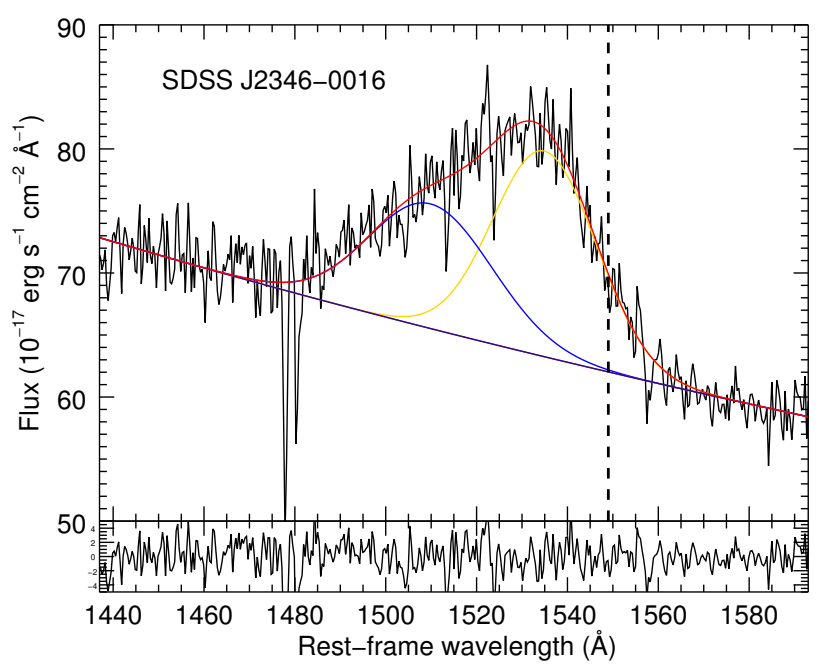

Fig. C.18. Same legend as in Fig. C.1. 